\title{
International Union of Basic and Clinical Pharmacology. XCV. Recent Advances in the Understanding of the Pharmacology and Biological Roles of Relaxin Family Peptide Receptors 1-4, the Receptors for Relaxin Family Peptides
}

\author{
Michelle L. Halls, Ross A. D. Bathgate, Steve W. Sutton, Thomas B. Dschietzig, and Roger J. Summers
}

Drug Discovery Biology, Monash Institute of Pharmaceutical Sciences, Parkville, Victoria, Australia (M.L.H., R.J.S.); Neuropeptides Division, Florey Institute of Neuroscience and Mental Health and Department of Biochemistry and Molecular Biology, University of Melbourne, Parkville, Victoria, Australia (R.A.D.B.); Neuroscience Drug Discovery, Janssen Research \& Development, LLC, San Diego, California (S.W.S.); Immundiagnostik AG, Bensheim, Germany (T.B.D.); and Charité-University Medicine Berlin, Campus Mitte, Medical Clinic for Cardiology and Angiology, Berlin, Germany (T.B.D.)

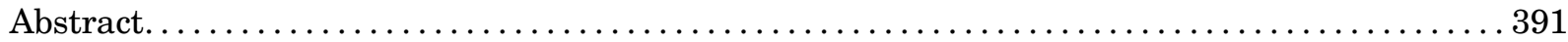

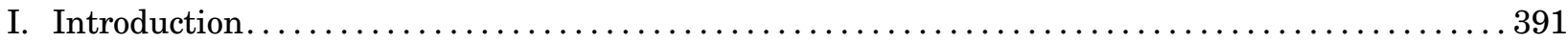

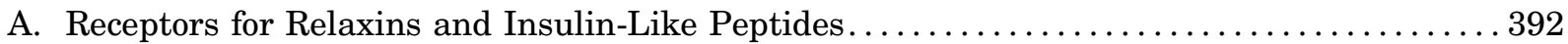

1. Relaxin Family Peptide Receptors 1 and 2-The Leucine-Rich Repeat-Containing

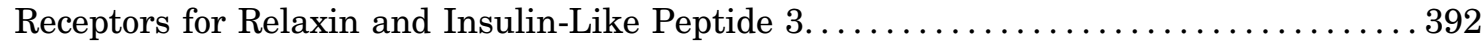

2. Relaxin Family Peptide Receptors 3 and 4-Receptors for Relaxin-3 and Insulin-Like Peptide 5 That Resemble G Protein-Coupled Receptors Activated by Small Peptides

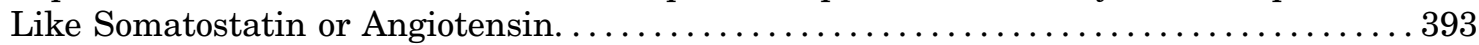

B. Structural Features of Relaxin Family Peptide Receptors ...................... 393

1. Functional Domains of Relaxin Family Peptide Receptors 1 and $2 \ldots \ldots \ldots \ldots \ldots \ldots 39$

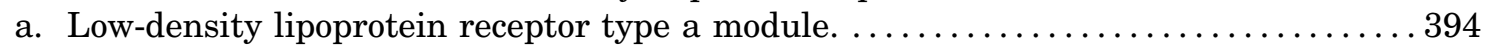

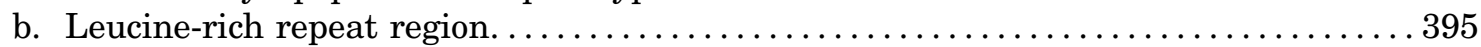

c. Transmembrane domains and dimerization. . . . . . . . . . . . . . . . . . 396

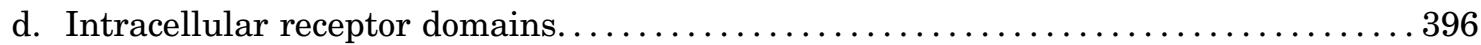

2. Functional Domains of Relaxin Family Peptide Receptors 3 and $4 \ldots \ldots \ldots \ldots \ldots \ldots 7$

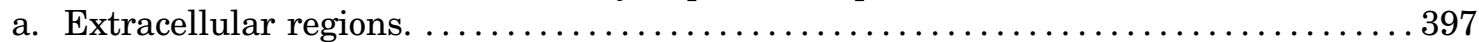

b. Transmembrane spanning regions. .................................. 398

II. Ligands That Act at Relaxin Family Peptide Receptors. . . . . . . . . . . . . . . . . . . . . . 398

A. Ligands That Act at Relaxin Family Peptide Receptor $1 \ldots \ldots \ldots \ldots \ldots \ldots \ldots \ldots \ldots \ldots$

1. Relaxin Family Peptide Agonists.................................... 399

2. C1q-Tumor Necrosis Factor-Related Protein 8: An Additional Relaxin Family

Peptide Receptor 1 Ligand?........................................ 399

3. Small-Molecular-Weight Agonists. ................................. 400

4. Relaxin Family Peptide Receptor 1 Antagonists. ......................... 401

B. Ligands That Act at Relaxin Family Peptide Receptor $2 \ldots \ldots \ldots \ldots \ldots \ldots \ldots \ldots \ldots \ldots \ldots 402$

1. Relaxin Family Peptide Agonists.................................... 402

2. Relaxin Family Peptide Antagonists............................... 402

M.L.H. was supported by the National Health and Medical Research Council (NHMRC) of Australia Project Grant 1047633 and RD Wright Career Development Fellowship 1061687. R.A.D.B. was supported by NHMRC Project Grants 628427 and 1043750 , by the Victorian Government Operational Infrastructure Support Program, and by a NHMRC Research Fellowship. R.J.S. was supported by NHMRC Program Grants 519461 and 1055134. T.B.D. was supported by Grants 89874634 and 89844575 from BAS Medical Inc. and Grants KF2181501AJ9 and KF2318301 from Zentrales Innovationsprogramm Mittelstand. NC-IUPHAR is supported in part by Wellcome Trust Grant 099156/Z/12/Z.

Address correspondence to: Dr. Roger Summers, Drug Discovery Biology, Monash Institute of Pharmaceutical Sciences, 399 Royal Parade, Parkville, VIC 3052, Australia. E-mail roger.summers@monash.edu

dx.doi.org/10.1124/pr.114.009472. 
C. Ligands That Act at Relaxin Family Peptide Receptor $3 \ldots \ldots \ldots \ldots \ldots \ldots \ldots \ldots \ldots \ldots 403$

1. Relaxin Family Peptide Agonists..................................... 403

2. Biased Agonists Acting at Relaxin Family Peptide Receptor $3 \ldots \ldots \ldots \ldots \ldots \ldots \ldots \ldots \ldots 403$

3. Allosteric Modulators. .......................................... 403

4. Relaxin Family Peptide Receptor 3 Antagonists. ......................... 403

D. Ligands That Act at Relaxin Family Peptide Receptor $4 \ldots \ldots \ldots \ldots \ldots \ldots \ldots \ldots \ldots \ldots \ldots 4$

1. Relaxin Family Peptide Agonists..................................... 404

2. Modified Peptide Agonists. ........................................... 405

3. Relaxin Family Peptide Receptor 4 Antagonists. ........................ 405

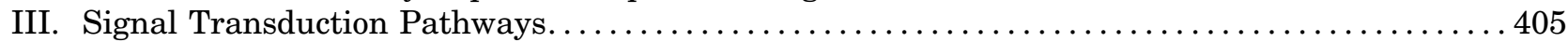

A. Relaxin Family Peptide Receptor 1 Signaling. ............................. 405

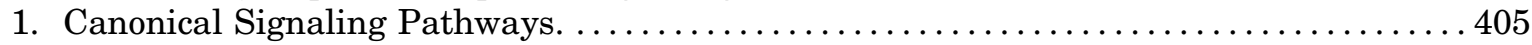

2. Noncanonical Signaling Pathways................................... 408

3. The Relaxin Family Peptide Receptor 1 Signalosome-A Protein Complex That

Facilitates High-Sensitivity Signaling in Response to Relaxin.................. 409

4. Homo- and Hetero-Oligomerization of RXFP1 and Its Functional Consequences. . . . . 410

5. Allosteric Agonist Signaling at Relaxin Family Peptide Receptor $1 \ldots \ldots \ldots \ldots \ldots \ldots . \ldots 41$

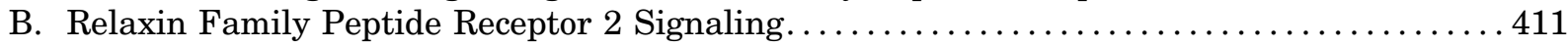

1. Canonical Signaling Pathways. ....................................... 411

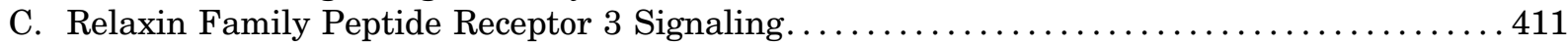

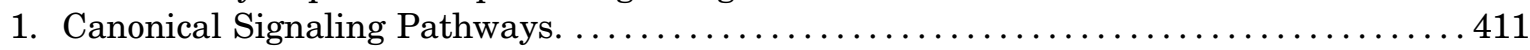

2. Ligand-Directed Signaling Bias at Relaxin Family Peptide Receptor $3 \ldots \ldots \ldots \ldots \ldots 412$

3. Allosteric Modulation of Relaxin Family Peptide Receptor $3 . \ldots \ldots \ldots \ldots \ldots \ldots \ldots \ldots 414$

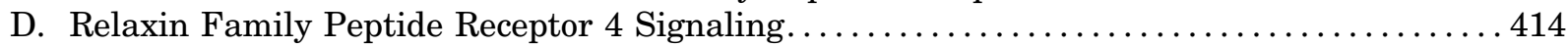

1. Canonical Signaling Pathways. ....................................... 414

IV. Receptor-Protein Signaling and Regulatory Complexes $\ldots \ldots \ldots \ldots \ldots \ldots \ldots \ldots \ldots \ldots \ldots \ldots 414$

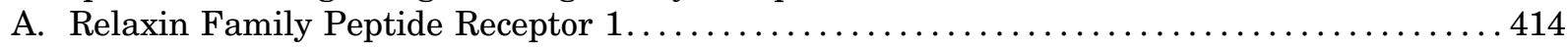

1. G Proteins That Couple to Relaxin Family Peptide Receptor $1 \ldots \ldots \ldots \ldots \ldots \ldots \ldots \ldots 414$

2. Interactions between Relaxin Family Peptide Receptor 1 and $\beta$-Arrestins. ........ 415

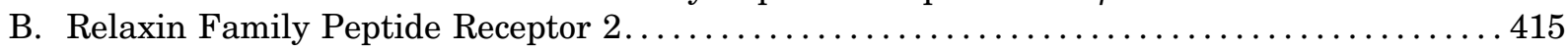

1. G Proteins That Couple to Relaxin Family Peptide Receptor $2 \ldots \ldots \ldots \ldots \ldots \ldots \ldots \ldots 45$

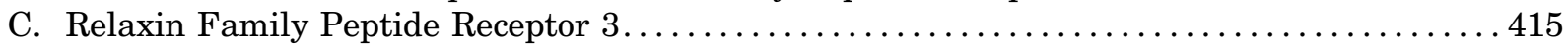

1. G Proteins That Couple to Relaxin Family Peptide Receptor 3 after Activation by Human Relaxin-3............................................... 415

2. G Proteins That Couple to Relaxin Family Peptide Receptor 3 after Activation by the

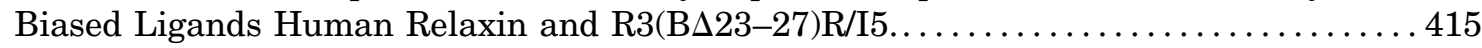

3. Interactions between Relaxin Family Peptide Receptor 3 and $\beta$-Arrestins. .......... 416

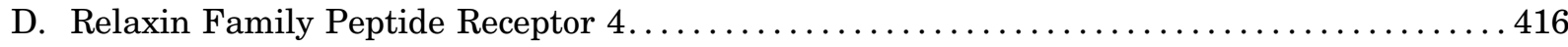

1. G Proteins That Couple to Relaxin Family Peptide Receptor $4 \ldots \ldots \ldots \ldots \ldots \ldots \ldots \ldots 416$

V. Physiologic Roles of Relaxin Family Peptide Receptors and Their Cognate Ligands ......... 416

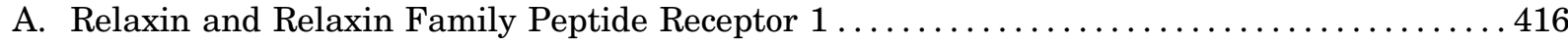

1. Reproduction. .................................................. 416

2. Central Nervous System. ........................................ 417

3. Blood Vessels.................................................. 417

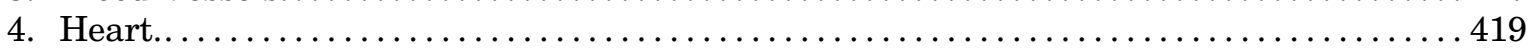

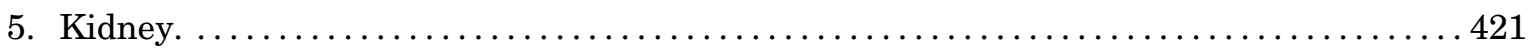

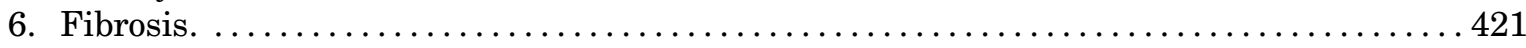

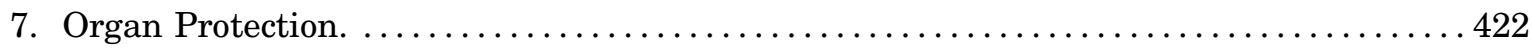

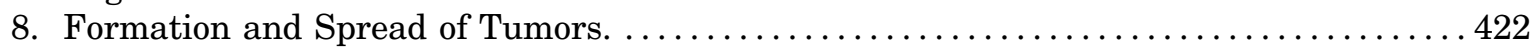

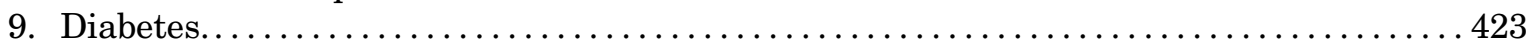

B. Insulin-Like Peptide 3 and Relaxin Family Peptide Receptor $2 \ldots \ldots \ldots \ldots \ldots \ldots \ldots \ldots 423$

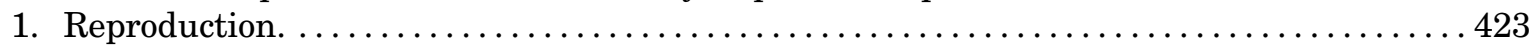

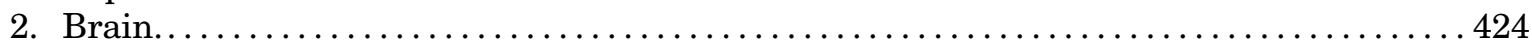

3. Bone and Other Tissues......................................... 424

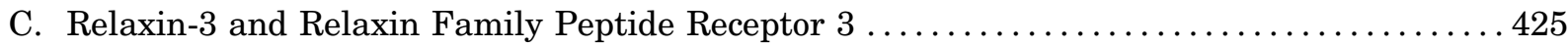

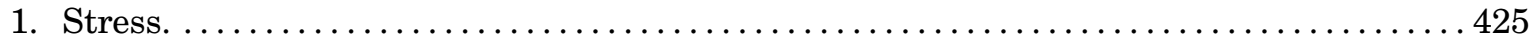

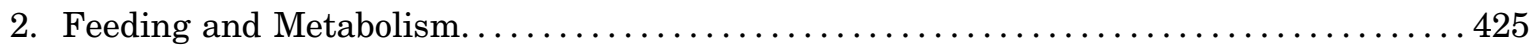


3. Behavioral Activation and Arousal. .................................. 426

D. Insulin-Like Peptide 5 and Relaxin Family Peptide Receptor $4 \ldots \ldots \ldots \ldots \ldots \ldots \ldots \ldots 27$

1. Metabolism................................................ 427

VI. Unresolved Pharmacological and Therapeutic Issues and Future Directions.............428

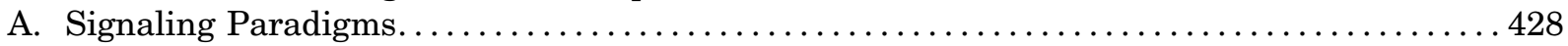

1. Heterodimerization of Relaxin Family Peptide Receptor 1: A Possible Explanation for the Selective Actions of Relaxin in Diseased or Pathologic States?............. 428

2. The Development of ML290, a Small-Molecular-Weight Allosteric Agonist at Relaxin

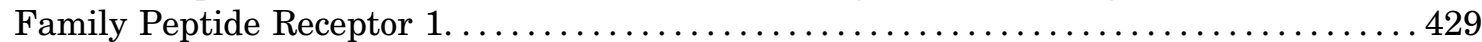

3. Novel Activators of RXFP Receptors................................. 429

B. Therapeutic Applications . ...................................... 430

1. Is There Therapeutic Potential in Small-Molecule Targeting of Relaxin Family Peptide Receptor 1 Heterodimers? .................................... 430

2. Relaxin Family Peptide Receptor 3 as a Drug Target. ..................... 430

3. Relaxin Family Peptide Receptors and Metabolism. ...................... 431

References ................................................... 431

\begin{abstract}
Relaxin, insulin-like peptide 3 (INSL3), relaxin-3, and INSL5 are the cognate ligands for the relaxin family peptide (RXFP) receptors $1-4$, respectively. RXFP1 activates pleiotropic signaling pathways including the signalosome protein complex that facilitates highsensitivity signaling; coupling to $G \alpha_{s}, G \alpha_{i}$, and $G \alpha_{o}$ proteins; interaction with glucocorticoid receptors; and the formation of hetero-oligomers with distinctive pharmacological properties. In addition to relaxinrelated ligands, RXFP1 is activated by Clq-tumor necrosis factor-related protein 8 and by small-molecularweight agonists, such as ML290 [2-isopropoxy- $N$-(2-(3(trifluoromethylsulfonyl)phenylcarbamoyl)phenyl) benzamide], that act allosterically. RXFP2 activates only the $G \alpha_{\mathrm{s}^{-}}$and $G \alpha_{\mathrm{o}}$-coupled pathways. Relaxin-3 is primarily
\end{abstract}

\section{Introduction}

Relaxin family peptides are heterodimeric and closely related structurally to insulin. Four of these peptides, relaxin, insulin-like peptide 3 (INSL3), relaxin-3, and insulin-like peptide 5 (INSL5) are the cognate ligands for the relaxin family peptide receptors RXFP1-4, respectively a neuropeptide, and its cognate receptor RXFP3 is a target for the treatment of depression, anxiety, and autism. A variety of peptide agonists, antagonists, biased agonists, and an allosteric modulator target RXFP3. Both RXFP3 and the related RXFP4 couple to $G \alpha_{i} / G \alpha_{o}$ proteins. INSL5 has the properties of an incretin; it is secreted from the gut and is orexigenic. The expression of RXFP4 in gut, adipose tissue, and $\beta$-islets together with compromised glucose tolerance in INSL5 or RXFP4 knockout mice suggests a metabolic role. This review focuses on the many advances in our understanding of RXFP receptors in the last 5 years, their signal transduction mechanisms, the development of novel compounds that target RXFP1-4, the challenges facing the field, and current prospects for new therapeutics.

ABBREVIATIONS: 135PAM1, 3-[3,5-bis(trifluoromethyl)phenyl]-1-(3,4-dichlorobenzyl)-1-[2-(5-methoxy-1H-indol-3-yl)ethyl]urea; AC, adenylyl cyclase; AKAP79, A-kinase anchoring protein 79; $\mathrm{AT}_{2} \mathrm{R}$, angiotensin type 2 receptor; BQ123, 2-[(3R,6R,9S,12R,15S)-6-(1H-indol-3-ylmethyl)-9-(2methylpropyl)-2,5,8,11,14-pentaoxo-12-propan-2-yl-1,4,7,10,13-pentazabicyclo[13.3.0]octadecan-3-yl]acetic acid; BRET, bioluminescence resonance energy transfer; CNS, central nervous system; CRF, corticotropin releasing factor; CTRP8, C1q-tumor necrosis factor-related protein 8; D06, bis(4- $N, N$ dimethylaminophenyl)(2-chloro-5-nitrophenyl) methane; ECL, extracellular loop; ELISA, enzyme-linked immunosorbant assay; eNOS, endothelial nitric oxide synthase; ERK1/2, extracellular signal-regulated kinase; ET, endothelin; GLP-1, glucagon-like peptide 1; GM6001, (2R)- $N$ '-hydroxy- $N$-[(2S)-3-(5Hindol-3-yl)-1-methylamino-1-oxopropan-2-yl]-2-(2-methylpropyl)butanediamide; GPCR, G protein-coupled receptor; GR, glucocorticoid receptor; 5-HT, 5hydroxytryptamine; ICL, intracellular loop; IL, interleukin; iNOS, inducible nitric oxide synthase; INSL3, insulin-like peptide 3; INSL5, insulin-like peptide 5; IR, ischemia-reperfusion; LDLa, low-density lipoprotein receptor type A module; LRR, leucine-rich repeat; LY294002, 2-(4-morpholinyl)-8phenyl-1(4H)-benzopyran-4-one hydrochloride; MAPK, mitogen-activated protein kinase; ML290, 2-isopropoxy- $N$-(2-(3-(trifluoromethylsulfonyl)phenylcarbamoyl)phenyl)benzamide; MMP, matrix metalloproteinase; MS, medial septum; NF449, 4,4',4",4"''-[carbonylbis(imino-5,1,3-benzenetriyl-bis (carbonylimino))]tetrakis-1,3-benzenedisulfonic acid, octasodium salt; $\mathrm{NF} \kappa \mathrm{B}$, nuclear factor- $\kappa \mathrm{B}$; NI, nucleus incertus; NO, nitric oxide; NOS, nitric oxide synthase; nNOS, neuronal nitric oxide synthase; OVLT, organum vasculosum of the lamina terminalis; PAM, positive allosteric modulator; pERK1/2, phosphorylated ERK1/2; PD123319, $S$-(+)-1-[(4-(dimethylamino)-3-methylphenyl)methyl]-5-(diphenylacetyl)-4,5,6,7-tetrahydro-1H-imidazo[4,5-c]pyridine-6-carboxylic acid di(trifluoroacetate); PD98059, 2'-amino-3'-methoxyflavone; PDE, phosphodiesterase; PGF, placental growth factor; PI3K, phosphatidylinositol 3-kinase; PKA, protein kinase A; PKC, protein kinase C; PTX, pertussis toxin; PVN, paraventricular nucleus of the hypothalamus; RU-486, 17 $\beta$-hydroxy-11 $\beta$-(4-dimethylamino-phenyl)- 17 $\alpha$-(1-propinyl)estra-4,9-dien-3-on; RWJ67657, 4-[4-(4-fluorophenyl)-1-(3-phenylpropyl)-5-(4-pyridinyl)-1H-imidazol-2-yl]-3-butyn-1-ol; RXFP1-4, relaxin family peptide receptor 1-4; SB209670, (+)-(1S,2R,3S)-3-(2-carboxymethoxy4-methoxyphenyl)1-(3,4-methylenedioxyphenyl)-5-(prop-1-yloxy)indane-2-carboxylic acid; SFO, subfornical organ; SOD, superoxide dismutase; SP600125, anthra[1,9-cd] pyrazol-6(2H)-one; SU5416, (3Z)-3-[(3,5-dimethyl-1H-pyrrol-2-yl)methylidene]-1,3-dihydro-2H-indol-2-one; T2DM, type-2 diabetes mellitus; TGF, transforming growth factor; TIMP, tissue inhibitor of metalloproteinase; TM, transmembrane; VEGF, vascular endothelial growth factor; WT, wild type. 
as relaxin). The function of the $R L N 1$ gene in humans and higher primates is unknown (relaxin-1). In humans, the relaxin family peptide genes have a similar structure and all synthesize prepropeptides (Hsu, 2003) that are processed by convertases to produce the mature twochain peptides; an A-chain linked to a B-chain by two disulfide bonds, and an additional intrachain disulfide in the A-chain.

Relaxin circulates in the blood of all mammals during pregnancy, although the levels and roles vary widely in different species. In humans, relaxin levels are highest in the first trimester and are probably associated with implantation and initiation of the cardiovascular changes that accompany pregnancy. However, relaxin is also produced in many tissues in both male and female mammals as a paracrine or autocrine factor to exert other physiologic roles (Sherwood, 2004; Bathgate et al., 2006a,c, 2013a).

Relaxin-3 is the most recently identified relaxin family peptide; it was named as a "relaxin" peptide because of the presence of the characteristic RxxxRxxI/V relaxinbinding motif in the B-chain but otherwise has relatively low sequence homology to other relaxin peptides. In contrast to other relaxins, the sequence of relaxin-3 is well conserved across species (Wilkinson et al., 2005b; Yegorov et al., 2009). Relaxin-3 is believed to be the ancestral peptide of the family (Wilkinson et al., 2005b) and in mammals is primarily a neuropeptide (Bathgate et al., 2002) involved in stress, memory, and appetite regulation (McGowan et al., 2005; Tanaka et al., 2005; Ma et al., 2007a; Banerjee et al., 2010; Ganella et al., 2013a,b; Ryan et al., 2013a,b; Smith et al., 2014).

INSL3 (formerly Leydig insulin-like peptide) was discovered in the Leydig cells of the testis (Adham et al., 1993) where it is highly expressed in all species that have the INSL3 gene (Bathgate et al., 2006c). INSL3 expression in other tissues occurs at much lower levels. INSL3 has a critical role in testis descent, and INSL3 knockout mice are cryptorchid and infertile (Nef and Parada, 1999; Zimmermann et al., 1999). It plays an important role in gubernaculum development, which is involved in the first stage of testis descent, and also appears to have a role in the maintenance of ovarian function (Spanel-Borowski et al., 2001; Kawamura et al., 2004; Glister et al., 2013).

INSL5 is widely distributed with high expression in the gastrointestinal tract (Conklin et al., 1999) particularly in L cells isolated from mouse colon/rectum but also in ascending, transverse, and descending colon and proximal rectum, with lower levels in the cecum and distal rectum (Grosse et al., 2014). Low levels of Insl5 mRNA were found in the pancreas, thymus, and eye (Grosse et al., 2014). INSL5 knockout mice display dysfunctional glucose homeostasis (Burnicka-Turek et al., 2012). INSL5 activates RXFP4, but not RXFP1 or RXFP2, with high potency and is a weak antagonist at RXFP3 (Liu et al., 2005b).
Thus, although relaxin peptides resemble each other closely in structure, each is the cognate ligand for a specific G protein-coupled receptor (GPCR) and each possesses a wide variety of physiologic functions. Relaxin has roles in reproduction, cardiovascular system, organ protection, metabolism, and as a neuropeptide in the brain; INSL3, although acting on a similar receptor, has highly specialized roles in reproduction; relaxin-3 is a neuropeptide, and INSL5 acts as an incretin.

\section{A. Receptors for Relaxins and Insulin-Like Peptides}

1. Relaxin Family Peptide Receptors 1 and 2-The Leucine-Rich Repeat-Containing Receptors for Relaxin and Insulin-Like Peptide 3. Early studies showed an increase in tyrosine phosphorylation of a $220-\mathrm{kDa}$ protein in response to relaxin (Palejwala et al., 1998), suggesting that relaxin receptors, like those that respond to insulin, were tyrosine kinases. However, Insl3 knockout mice (Nef and Parada, 1999; Zimmermann et al., 1999) displayed abnormal testis descent as did mice with disruptions in the GPCR encoded by the GREAT gene (later shown to be the mouse ortholog of human LGR8 or RXFP2) (Overbeek et al., 2001). This led to the deorphanization of LGR7 (RXFP1) and LGR8 (RXFP2) (Hsu et al., 2002), two family A GPCRs.

In humans, RXFP1 is the cognate receptor for human relaxin; it has the classic seven-transmembrane (TM) spanning regions of a GPCR as well as a large extracellular domain containing 10 leucine-rich repeats (LRR) and a unique N-terminal low-density lipoprotein receptor type A (LDLa) module (Hsu et al., 2002). RXFP1 mRNA and protein is found in ovary, uterus, placenta, mammary gland, prostate, and testis but also in the heart, arteries, kidney, lung, liver, and blood cells as well as in a number of areas of the brain, such as cortex, hippocampus, arcuate nucleus, organum vasculosum of the lamina terminalis (OVLT), and subfornical organ (SFO) (for details, see Novak et al., 2006, and Bathgate et al., 2013a). Thus, relaxin, in addition to autocrine and paracrine roles, also acts as a neuropeptide. Interaction of relaxin with RXFP1 to trigger cell signaling involves at least three stages: high-affinity binding between the B-chain of relaxin and the RXFP1 LRR region, lower affinity binding to the TM extracellular loops (ECLs), and finally an essential interaction involving the LDLa module (Sudo et al., 2003; Halls et al., 2005b). Although RXFP1 couples to numerous signal transduction pathways, many early studies in reproductive tissues indicated that relaxin caused increases in cAMP levels (Braddon, 1978; Cheah and Sherwood, 1980; Sanborn et al., 1980; Chen et al., 1988), and a constitutively active receptor mutant also generates cAMP (Hsu et al., 2002). The pattern of cAMP production after stimulation of HEK293 cells expressing RXFP1 is complex and involves at least three G proteins (Halls et al., 2006, 2009a,b). RXFP1 also activates extracellular signal-regulated kinase 1/2 (ERK1/2), tyrosine kinase(s), gene transcription, 
and nitric oxide (NO) signaling, and relaxin interacts with the glucocorticoid receptor (GR). The full implications of the pleiotropic effects of relaxin are still to be elucidated.

RXFP2 is the cognate receptor for INSL3 and is structurally similar to RXFP1 (Hsu et al., 2002; Kumagai et al., 2002). It is primarily expressed in the ovary, testis, and gubernaculum (for details, see Bathgate et al., 2013a). It has a specialized role in the gubernaculum during testicular descent, it is expressed in the testis and ovary affecting gonadal function (Kawamura et al., 2004) and it influences bone metabolism (Ferlin et al., 2009). RXFP2 signaling also involves adenylyl cyclase (AC) activation and cAMP generation, utilizing a subset of the $\mathrm{G}$ proteins used by RXFP1. Although in vitro cell systems that are often used to study RXFP2 show increases in cAMP levels, in endogenously expressing systems both increases and decreases in cAMP may be seen. Thus, in gubernacular cells (Kumagai et al., 2002) or osteoblasts (Ferlin et al., 2009), activation of RXFP2 causes increased cAMP, but in male germ cells and oocytes, decreased cAMP is observed (Kawamura et al., 2004), perhaps reflecting expression patterns of signaling proteins in different cells (Halls et al., 2009a). Although the relaxins of some species activate RXFP2 in vitro (Hsu et al., 2002; Kumagai et al., 2002; Scott et al., 2005a; Bathgate et al., 2006b), there is no evidence that relaxin activates RXFP2 in vivo.

Thus, RXFP1 and RXFP2 are structurally similar receptors that use some common signaling mechanisms. However, current evidence suggests that the relaxin/ RXFP1 system has a much wider range of distribution and functions than the INSL3/RXFP2 system.

2. Relaxin Family Peptide Receptors 3 and 4-Receptors for Relaxin-3 and Insulin-Like Peptide 5 That Resemble $G$ Protein-Coupled Receptors Activated by Small Peptides Like Somatostatin or Angiotensin. RXFP3 and RXFP4 are the targets for relaxin-3 and INSL5, respectively (Liu et al., 2003a,b). They are distinctly different in structure from RXFP1 and RXFP2 and instead resemble the class A small peptide receptors. RXFP3 was originally named SALPR or somatostatin and angiotensin-like receptor (Matsumoto et al., 2000), whereas RXFP4 was thought to be a bradykinin receptor (Boels and Schaller, 2003) before pairing with their cognate ligands relaxin-3 (Liu et al., 2003b) and INSL5 (Liu et al., 2005b). The receptor expression profiles suggest that RXFP3 is a neuropeptide receptor and RXFP4 is a gut hormone receptor (Bathgate et al., 2013a; Grosse et al., 2014). RXFP3 couples to $\mathrm{G} \alpha_{\mathrm{i} / \mathrm{o}}$ to inhibit AC (Liu et al., 2003b; van der Westhuizen et al., 2007) and also causes ERK1/2 phosphorylation (van der Westhuizen et al., 2007). Based on binding and signaling studies, relaxin can also bind to a subtly different region on RXFP3 to activate a subset of the pathways activated by relaxin-3 (van der Westhuizen et al., 2010; Kocan et al., 2014). Relatively little is known about RXFP4 signaling, but like RXFP3 it couples to inhibitory G proteins.
RXFP3 and RXFP4 are closely related receptors with structurally similar cognate ligands. However, RXFP3 and relaxin-3 are predominantly involved in central nervous system neurotransmission, whereas INSL5 is an incretin that targets RXFP4 located in the gastrointestinal tract and tissues controlling metabolism.

\section{B. Structural Features of Relaxin Family Peptide Receptors}

The relaxin family peptide receptors are four highly conserved family A GPCRs that can be considered as two distinct pairs based on their architecture and signaling properties. Genes encoding RXFP1 and RXFP2 have introns giving rise to a large number of splice variants with functions that are mostly yet to be established (Muda et al., 2005; Kern et al., 2008), whereas genes encoding RXFP3 and RXFP4 are intronless. RXFP1 and RXFP2 have a large extracellular $\mathrm{N}$ terminus with an LRR domain containing a high-affinity ligand binding site complemented by a low-affinity binding interaction within ECL2 and uniquely, an N-terminal LDLa module that is essential for signaling, and a role in trafficking (Fig. 1). RXFP1 and RXFP2 show similarities in ligand binding and signaling, but only RXFP1 has a $\mathrm{C}$ terminus that contains motifs that induce the formation of protein signaling complexes termed signalosomes (see section III.A.3) (Halls and Cooper, 2010; Halls, 2012) (Fig. 1). The third intracellular loop (ICL3) is likely essential for G protein coupling in all RXFP receptors. Both RXFP1 and RXFP2 couple to $\mathrm{G} \alpha_{\mathrm{S}}$ and to inhibitory $\mathrm{G} \alpha_{\mathrm{oB}}$, which modulates the effect of $\mathrm{G} \alpha_{\mathrm{S}}$ coupling, but only RXFP1 is able to couple to $\mathrm{G} \alpha_{\mathrm{i} 3}$ to produce a delayed surge in cAMP accumulation that occurs in many but not all cell types (Halls et al., 2009a). In contrast, RXFP3 and RXFP4 have a very small N-terminal domain and couple solely to $\mathrm{G} \alpha_{\mathrm{i}}$ and $\mathrm{G} \alpha_{\mathrm{o}}$ proteins. For all receptors, the pattern of $\mathrm{G}$ protein coupling is dependent on the cell type in which the receptor is expressed.

1. Functional Domains of Relaxin Family Peptide Receptors 1 and 2. The similarity between RXFP1 and RXFP2 has helped the identification of the functional domains of the two receptors. Initial work used chimeric receptors and relaxin-3, because it is selective for RXFP1 over RXFP2, and revealed that the peptide interacts with both the LRR domain and ECL2 of the TM domain of RXFP1 to produce the full binding and cAMP signaling profile (Sudo et al., 2003) (Fig. 1). This mechanism was later shown to be a feature of both receptors (Halls et al., $2005 \mathrm{~b})$. Support for two binding sites also came from functional assays, with high-affinity LRR binding producing cAMP accumulation more effectively than the lower affinity ECL site (Halls et al., 2005b). In addition to the LRR region, the $\mathrm{N}$-terminal LDLa module is essential for signaling (Fig. 1) but has no role in ligand binding (Scott et al., 2006; Hopkins et al., 2007; Kern et al., 2007). The LDLa module likely interacts with other receptor domains (potentially the ECLs and TM domains) in a manner analogous to a tethered ligand (Fig. 1). 


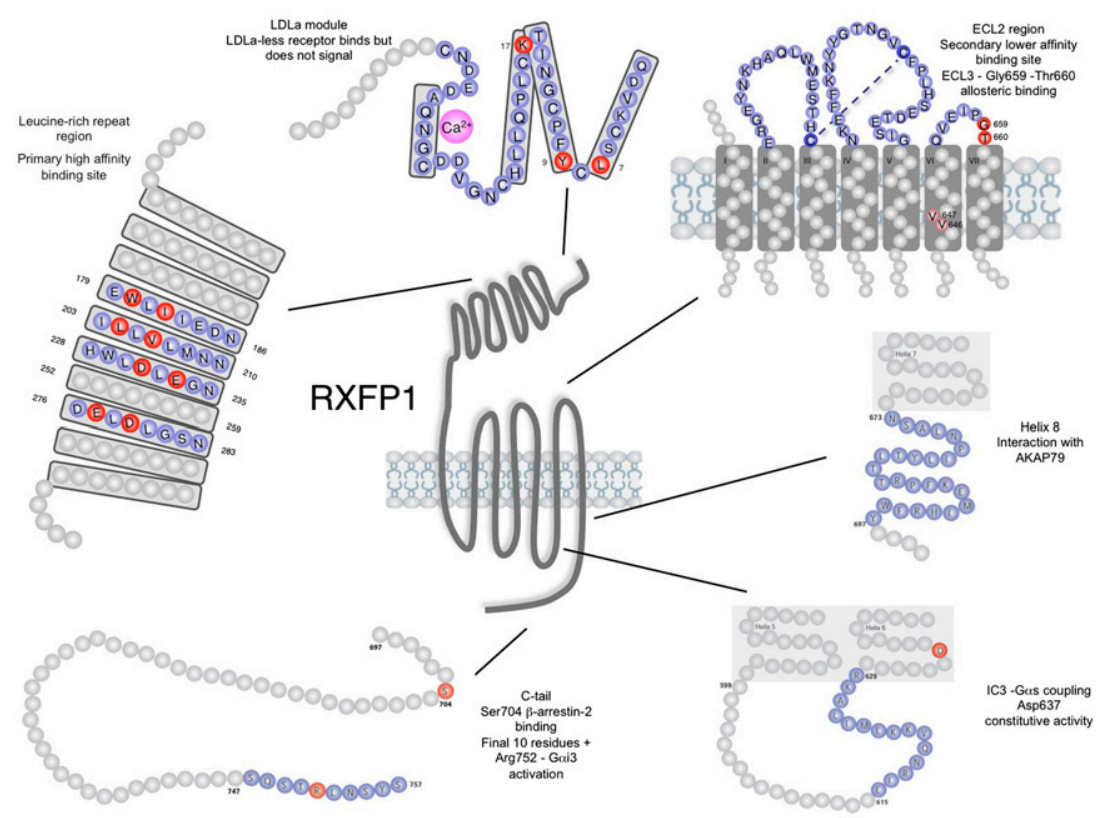

Fig. 1. Functional domains of RXFP1. The N-terminal region of RXFP1 consists of an LDLa module that is essential for signaling promoted by relaxin but not the allosteric modulator ML290. Residues L29, Y31, and K39 are important for receptor activation by relaxin. The LDLa module is connected to the leucine-rich repeat region that contains the primary high-affinity binding site for relaxin. The relaxin B-chain residues R13 and R17 are believed to bind to E277 and D279 in LRR8 and D231 and E233 in LRR6, whereas I20 is thought to interact with W180 and I182 in LRR4 and L204 and V206 in LRR5. The ECL2 region contains the secondary low-affinity binding site for relaxin, whereas ECL3 contains two residues, G659 and T660, that are essential for activation of RXFP1 by ML290. Helix 8 contains binding motifs that are essential for interaction with AKAP79 that is required for signalosome formation. The distal region of ICL3 is required for coupling to $\mathrm{G} \alpha_{\mathrm{s}}$ and the receptor containing D637 displays constitutive activity. In the C-terminal tail region S704 is required for $\beta$-arrestin binding and signalosome formation, whereas the final 10 residues and in particular R752 are required for coupling of RXFP1 to $\mathrm{G} \alpha \mathrm{i}$.

a. Low-density lipoprotein receptor type a module. A unique feature of RXFP1 and RXFP2 is the LDLa module, originally defined by the structure of the LDL receptor (Yamamoto et al., 1984; Daly et al., 1995) but now recognized in a number of other proteins including the very-low-density lipoprotein receptor (Gafvels et al., 1993), the LDL receptor-related protein (Herz et al., 1988), renal glycoprotein gp330 (Lundgren et al., 1994; Hjalm et al., 1996), the C9 component of complement (DiScipio et al., 1984; Stanley et al., 1985), the Tva receptor for Rous sarcoma virus (Bates et al., 1993), and retina- and brain-specific neuropilin and tolloid-like protein 1 transmembrane protein (Stohr et al., 2002). RXFP1 and RXFP2 are the only known human GPCRs to contain this module (Bathgate et al., 2006a). The NMR solution structure of the RXFP1 LDLa module has been solved and reveals a typical fold generated by six essential cysteine residues and the incorporation of a calcium ion by a largely conserved motif of acidic residues (Hopkins et al., 2007). The role of the LDLa module was first discovered during characterization of a splice variant of RXFP2 that is missing the LDLa module. This receptor and a similar RXFP1 mutant were expressed at the cell surface and bound their native ligands normally but were not able to signal through cAMP (Scott et al., 2006). More recent studies used a panel of GPCR-relevant signaling reporter genes to demonstrate that the RXFP1 receptor lacking the LDLa module does not signal through any pathway tested (Kong et al., 2013). Importantly, a soluble recombinant form of the RXFP1 LDLa module acts as an RXFP1 antagonist when introduced into cells expressing RXFP1 (Scott et al., 2005c, 2006). These data suggest that the LDLa module may act as an RXFP1 tethered ligand and its actions can be blocked by the addition of recombinant LDLa. Further mutagenesis studies have provided evidence of a specific LDLa-RXFP1 interaction that drives receptor activation. Mutation of residues involved in the folding of the LDLa module produce mutants that are unable to signal but maintain intact ligand binding profiles (Scott et al., 2006; Hopkins et al., 2007; Kern et al., 2007) (Fig. 1). Specifically, mutation of cysteines (C47A and C53A) involved in folding or of D58E involved in calcium-binding produces RXFP1 receptors that are unable to increase cAMP accumulation in response to relaxin (Kern et al., 2007). Other mutations that affect calcium ligation and hence folding (corresponding to $\mathrm{C} 27 \mathrm{~S}$ and C40S within the full-length RXFP1 sequence) also abolish signaling (Hopkins et al., 2007) (Fig. 1). Similar studies at human RXFP2 with mutations of the calcium ligating residue D70Y or conserved cysteine $\mathrm{C} 71 \mathrm{Y}$ also resulted in a loss of signaling, again highlighting the common mechanism of activation of the two receptors (Bogatcheva et al., 2007).

Further evidence for a specific interaction between the LDLa module and RXFP1 that drives receptor activation comes from studies on chimeric and mutant RXFP1 receptors (Hopkins et al., 2007; Kong et al., 2013). A chimera where the RXFP1 LDLa module is swapped 
for a homologous LDLa module from the second ligandbinding domain of the LDL receptor produces a receptor (LB2-RXFP1) that is unable to signal in response to relaxin but binds ligand normally (Hopkins et al., 2007). Mutagenesis of specific conserved residues in the RXFP1 LDLa module highlighted potential roles for L29 and Y31 in receptor activation (Hopkins et al., 2007). Further studies have used the signaling-deficient LB2-RXFP1 receptor to define the potential "signaling surface" of the LDLa module. Within the signaling-deficient LB2-RXFP1, gain of function mutations, loss of function mutations, and judicious amino acid substitutions were able to confirm the key role of L29 and Y31 and to additionally identify K39 as important residues in the RXFP1 LDLa module that are involved in the hydrophobic interactions that drive the active receptor conformation (Kong et al., 2013) (Fig. 1). Studies using chimeric RXFP1 and RXFP2 receptors with their LDLa modules swapped C-terminally to the final cysteine residue of the module demonstrate that RXFP2 likely uses a similar mechanism to drive receptor activation. Importantly the chimeric receptors were still able to bind ligand normally and were also able to signal, albeit with altered activity compared with wild-type receptors (Bruell et al., 2013). Furthermore, when the TM domains of the RXFP1 chimera with an RXFP2 LDLa (RXFP211) were swapped to match the LDLa module (RXFP212) the activity of the receptor approached wild type, suggesting a specific interaction between the LDLa module and the receptor TM domains. It also highlights that the RXFP1 and RXFP2 LDLa modules function by similar but distinct mechanisms.

The LDLa module may also be involved in receptor maturation and translocation to the cell surface. When expressed recombinantly in HEK293 cells, a large proportion of wild-type RXFP1 receptors exist in an immature form containing high mannose-type N-linked oligosaccharides within the endoplasmic reticulum (Kern et al., 2007). In the case of other glycoprotein hormone receptors, such as the leutenizing hormone receptor (Ascoli et al., 2002; Tao et al., 2004; Pietila et al., 2005) and follicle-stimulating hormone receptor (Quintana et al., 1993; Davis et al., 1995), this has been suggested to be a mechanism controlling cell surface expression. In the same study it was demonstrated that an RXFP1 mutant lacking the LDLa module was expressed as the mature form only, as was a chimeric RXFP1 receptor containing the LDLa module of RXFP2 (Kern et al., 2007). In addition, mutation of a conserved glycosylation site within the LDLa module results in a receptor with a reduced ability to generate cAMP, attributed to a decrease in cell surface expression (Kern et al., 2007; Yan et al., 2008). However, similar studies on RXFP1 mutants with a disruption of the LDLa glycosylation site show only a minor (Yan et al., 2008) or no effect (Kong et al., 2013) on signaling and cell surface expression. Additionally, studies using LDLaless RXFP1 and RXFP2 (Scott et al., 2006), LB2-RXFP1 mutants, RXFP1 LDLa loss of function mutants (Kong et al., 2013), or misfolded RXFP1 LDLa mutants (Hopkins et al., 2007) demonstrated no effect on cell surface expression. In RXFP2, mutations of amino acid residues that form the disulfide bond or coordinate calcium binding in the LDLa module (C70Y and D71Y) did reduce cellsurface expression (Bogatcheva et al., 2007).

The LDLa module clearly plays an important and specific role in protein maturation, cell surface expression, and in the activation of both RXFP1 and RXFP2.

b. Leucine-rich repeat region. Glycosylation, a posttranslational modification common to many GPCRs, is important for receptor delivery to the cell surface, ligand binding, and signal transduction. In addition to sites within the LDLa module, the LRR region is also glycosylated at a number of residues (N105, N250, N303, and N346), all of which are important for the translocation of receptors to the cell surface and full signaling efficacy but not ligand binding (Yan et al., 2008). The glycosylation status of RXFP2 has yet to be studied in detail.

In addition to a role in cell surface delivery, the LRR region is essential for ligand binding and signal transduction. Similar to other members of the LGR family, the primary high-affinity ligand binding site for relaxin and INSL3 is in the LRR region of RXFP1 and RXFP2 (Sudo et al., 2003; Halls et al., 2005b). Studies based on peptide mutagenesis and radioligand binding for both relaxin and INSL3 (outlined in sections II.A.1 and II.B.1) highlight that B-chain residues of the peptides contribute most to the binding affinity. Subsequently, two independent studies used homology modeling of the LRRs and mutagenesis to define the relaxin and INSL3 binding sites in RXFP1 and RXFP2, respectively. The RXFP1 LRRs were modeled based on the crystal structure of the porcine ribonuclease inhibitor (a protein with LRRs) together with in silico peptide docking and targeted receptor mutagenesis. Relaxin used the well characterized RxxxRxxI binding motif within the peptide B-chain at a $45^{\circ}$ angle across five of the parallel LRRs (Bullesbach and Schwabe, 2005b). Hence the relaxin B-chain residues R13 and R17 were predicted to interact with acidic groups within the concave face of the LRRs (E277 and D279 in LRR8 and D231 and E233 in LRR6) (Bullesbach and Schwabe, 2005b) (Fig. 1). The B-chain I20 was predicted to form a hydrophobic interaction with W180 and I182 within LRR4 and L204 and V206 within LRR5 (Bullesbach and Schwabe, 2005b) (Fig. 1).

The interaction of INSL3 with RXFP2 uses different residues in the B-chain as determined from peptide studies (see section II.B.1). Modeling of the INSL3 interaction with the RXFP2 LRRs used the NMR solution structure of INSL3 (Rosengren et al., 2006b) and a molecular model of the RXFP2 LRR based upon the crystal structure of the Nogo receptor (which shares higher amino acid sequence homology than the porcine ribonuclease inhibitor; Scott et al., 2007). With the use of a combination of peptide and LRR mutants in conjunction with in silico docking of the INSL3 B-chain to the LRR of RXFP2, seven residues within RXFP2 that potentially make contact with INSL3 
were defined. The $\mathrm{B}$-chain residue $\mathrm{R} 16$ was predicted to interact with RXFP2 D227, H12 with RXFP2 W177, V19 with RXFP2 I179, R20 with RXFP2 E229 and D181, and W27 with RXFP2 F131 and Q133 (Scott et al., 2007). Interestingly, although five of the RXFP2 residues that potentially interact with INSL3 are also found in RXFP1, the affinity of INSL3 for RXFP1 is very low (Sudo et al., 2003; Halls et al., 2005b). Another study that investigated this apparent contradiction used the RXFP1 extracellular domain construct linked to a single CD8 TM domain, termed 7BP (Hsu et al., 2002). This construct has a high affinity for INSL3 (Halls et al., 2005b) and was used as a template to further explore the interaction of INSL3 and the RXFP1 LRRs (Scott et al., 2012). Only four amino acid changes were necessary to obtain the equivalent RXFP2 affinity for INSL3 in the RXFP1 LRRs, but when these mutations were inserted into full-length RXFP1 there was only a modest gain of function for INSL3. This, in conjunction with previous studies (see section I.B.1.c), confirmed an important role for the TM domains in modulating ligand binding. Molecular modeling studies demonstrated that relaxin and INSL3 have distinct orientations of binding to the LRRs. Taken together the results suggest that there are critical differences both in the extracellular domain binding and the coordination of this binding with the TM binding site between RXFP1 and RXFP2 (Scott et al., 2012). Relaxin clearly binds to RXFP2 in a different manner from RXFP1 and also in a different manner from INSL3 binding to RXFP2.

c. Transmembrane domains and dimerization. The information above highlights the essential coordination of the LRR binding site with the binding site in the TM domains. As mentioned earlier, studies using chimeric receptors and the selectivity of relaxin-3 for RXFP1 versus RXFP2 suggest that this additional binding site is in ECL2 (Sudo et al., 2003) (Fig. 1). The presence of this additional binding site within the TM was confirmed using the same chimeric receptors together with relaxin peptides that have selectivity for RXFP1 or RXFP2 (rat relaxin, RXFP1 > RXFP2; INSL3, RXFP2 > RXFP1) (Halls et al., 2005b). Modeling studies suggest that A-chain residues in relaxin and INSL3 interact with this potential binding site (Hartley et al., 2009), but this has yet to be fully characterized. The current model of activation of RXFP1 and RXFP2 suggests that ligand binding to the LRRs and TM ECLs directs the LDLa to interact with the TM domain of a receptor homodimer partner to drive receptor activation (Kong et al., 2010; Bruell et al., 2013). However this model still requires experimental verification using site-directed mutagenesis or other methods that prevent dimer formation.

As with many other class A GPCRs, RXFP1 and RXFP2 form homo- and heterodimers (Kern et al., 2008; Svendsen et al., 2008a,b). Dimerization occurs in the absence of, and is independent, of ligand occupation of the receptor (Svendsen et al., 2008a,b). Heterodimers also form between the haloreceptor and a number of splice variants and are present at all stages of receptor translocation from the endoplasmic reticulum to the plasma membrane (Kern et al., 2008). Dimerization can also occur with a TM-only domain receptor (Svendsen et al., 2008a,b), highlighting that the TM domains likely drive dimerization as with other GPCRs. The authors suggested that the receptor ecotodomains could be required for stabilization of the dimer because of the lower dimerization efficiency observed for the TM-only receptors. However, a relatively poor expression of the TM-only receptors could also explain these results (R. A. D. Bathgate, unpublished data).

There is evidence to suggest that dimerization is associated with negative cooperativity (Svendsen et al., 2008a,b), whereby the affinity of unoccupied receptor binding sites progressively decreases as receptor occupancy increases. Two consequences of this are an increase in the functional concentration range of the ligand and a decrease in ligand residence time corresponding to an increase in free ligand concentration, potentially allowing selective activation of different signaling pathways (Shymko et al., 1997). This may provide one explanation for the observation that in many experimental and clinical situations the concentration-response curves for relaxin acting at RXFP1 are bell shaped (also see section III.A.4).

d. Intracellular receptor domains. There is evidence that ICL3 and the C-terminal tail have important roles in signaling. ICL3 has an important role in coupling RXFP1 to $\mathrm{G} \alpha_{\mathrm{s}}$ and is therefore essential for activation of AC and cAMP signaling (Fig. 1). Peptide fragments based on the N-terminal region of ICL3 [residues 615-629, and 619-629-Lys(Palm)] increased AC activity (Shpakov et al., 2007). These peptides functionally "antagonized" the cAMP response to relaxin activation of RXFP1 endogenously expressed in rat striatum and rat cardiac muscle (Shpakov et al., 2007). A synthetic peptide based on the $\mathrm{C}$ terminus of $\mathrm{G} \alpha_{\mathrm{s}}$ (residues 385-394) inhibited AC activity that was stimulated by relaxin or the ICL3 peptides (Shpakov et al., 2007), suggesting an interaction between $\mathrm{G} \alpha_{\mathrm{s}}$ and ICL3. This is a region that in many GPCRs interacts with G proteins (Kjelsberg et al., 1992; Ren et al., 1993; Herrick-Davis et al., 1997; Egan et al., 1998). ICL3 may also direct coupling to $\mathrm{G} \alpha_{\mathrm{oB}}$ for both RXFP1 and RXFP2, although direct evidence is lacking; however, truncation of the C-terminal tail of either receptor did not affect the $\mathrm{G} \alpha_{\mathrm{s}}$ or the $\mathrm{G} \alpha_{\mathrm{oB}}$ components of the cAMP signaling response, suggesting a common interaction site. Constitutive activation of RXFP1 involving $\mathrm{G} \alpha_{\mathrm{s}}$ (and $\mathrm{G} \beta \gamma$ ) activation of AC2 (Halls and Cooper, 2010) is also likely to depend upon $\mathrm{G} \alpha_{\mathrm{S}}$ coupling to ICL3 (see section III.A.3).

The C-terminal tail of RXFP1 has two clear roles in signaling; it controls cAMP accumulation through the $\mathrm{G} \alpha_{\mathrm{i} 3}$ pathway and is essential for signalosome formation (see sections III.A.1 and III.A.3). Only RXFP1 increases cAMP accumulation by coupling to $\mathrm{G} \alpha_{\mathrm{i} 3}$ (Halls et al., 
2006) and activation of AC5, utilizing a G $\beta \gamma$-phosphatidylinositol 3-kinase (PI3K)-protein kinase C (PKC) $\zeta$ pathway. Coupling of RXFP1 to this pathway involves the final 10 amino acids of the $\mathrm{C}$ terminus and absolutely requires $\mathrm{R} 752$; truncation of the $\mathrm{C}$ terminus or substitution of R752 selectively removes coupling to $\mathrm{G} \alpha_{\mathrm{i} 3}$ (Halls et al., 2009b) (Fig. 1). As yet the precise mechanism by which the terminal 10 residues of RXFP1 direct $\mathrm{G} \alpha_{\mathrm{i} 3}$ coupling is unclear. The possibilities include direct $\mathrm{G} \alpha_{\mathrm{i} 3}$ coupling (although not observed for any other GPCR), $\mathrm{G} \alpha_{\mathrm{i} 3}$ coupling after receptor phosphorylation, or recruitment of scaffolding proteins for colocalization of the receptor with $\mathrm{G} \alpha_{\mathrm{i} 3}$.

The C-terminal tail of RXFP1 also controls a small degree of constitutive activity (Halls and Cooper, 2010), and the receptor constitutively couples to AC2 via an association between helix 8 and A-kinase anchoring protein 79 (AKAP79). The protein complex facilitates $\mathrm{G} \alpha_{\mathrm{s}}$-and $\mathrm{G} \beta \gamma$-mediated stimulation of $\mathrm{AC} 2$ to cause cAMP accumulation in response to subpicomolar concentrations of relaxin. The amount of cAMP generated by this complex is tightly controlled by protein kinase $\mathrm{A}$ (PKA)-stimulated phosphodiesterase (PDE) 4D3, which is scaffolded to the receptor $\mathrm{C}$ terminus by an association between $\beta$-arrestin-2 and S704 of RXFP1 (Fig. 1). This protein complex, termed a signalosome, directed and maintained by the RXFP1 C-terminal tail, mediates a cAMP response to low concentrations of peptide and may provide a novel cellular response to low levels of relaxin in some physiologic situations.

The RXFP1 C-terminal tail also contains a number of potential consensus sequences for phosphorylation and protein-protein interactions and is the region of most variation between RXFP1 and the highly-related RXFP2 (Halls et al., 2007b). As such, it represents an area of functional divergence between the two receptors that may relate to the more varied physiologic roles of relaxin in relation to INSL3.

RXFP1 and RXFP2 therefore have similar structures, binding sites, and signaling mechanisms. Both receptors possess a high-affinity binding site in the LRR region, a lower affinity site in ECL2 of the TM domain, and require an intact LDLa module for signaling. RXFP1 has a more complex C-terminal region that contains residues essential for signaling involving $\mathrm{G} \alpha_{\mathrm{i} 3}$ and interactions with signalosomes. Both receptors form homo- and heterodimers, but the functional significance of this is unclear at present.

2. Functional Domains of Relaxin Family Peptide Receptors 3 and 4. RXFP3 and RXFP4 are classic peptide receptors of the Rhodopsin family of class A GPCRs and possess small N-terminal domains that are markedly different from those described for RXFP1 and RXFP2. Additionally, the relaxin-3 B-chain alone can bind and activate RXFP3, although with lower potency than the two-chain peptide, whereas both chains are required for interaction with RXFP1 (Kuei et al., 2007;
Hossain et al., 2008). Thus the mode of binding and activation differs from the relaxin/RXFP1 activation mechanism. Alanine scans show that R8, R12, R16, I15, and F20 in the B-chain central helix are required for human relaxin-3 binding, whereas R26 and W27 are essential for receptor activation (Kuei et al., 2007). The first study to investigate the interaction between relaxin-3 and the RXFP3 receptor used peptide selectivity for RXFP3 and RXFP4: relaxin-3 binds to and activates both RXFP3 and RXFP4, whereas INSL5 only activates RXFP4. Chimeric RXFP3 and RXFP4 receptors were used to identify potential functional roles for the extracellular domains and TM helices (Zhu et al., 2008; Bathgate et al., 2013a). Chimeras with swapped N-terminal domains suggested that the N terminus of RXFP4 is required for INSL5 and possibly relaxin-3 binding. Chimeras of RXFP3 with RXFP4 ECL1 or ECL3 domains demonstrated that these ECLs were not important for INSL5 binding to either receptor (Zhu et al., 2008). However, chimeras with swapped ECL2 domains revealed that ECL2 in RXFP3 and RXFP4 are required for ligand binding and possibly receptor activation. Furthermore, insertion of the $\mathrm{N}$ terminus and ECL2 of RXFP4 into RXFP3 produced a chimera with full INSL5 binding, demonstrating that these domains are necessary for INSL5 binding in RXFP4 (Zhu et al., 2008). However, this chimera did not increase GTP $\gamma \mathrm{S}$ binding, showing that these domains are not sufficient for receptor activation by INSL5.

a. Extracellular regions. A recent study performed comparative sequence analysis of multiple mammalian RXFP3 sequences, molecular modeling, and mutagenesis to map the relaxin-3 binding site in RXFP3 (Bathgate et al., 2013b). The truncation of the first 33 amino acids of the RXFP3 $\mathrm{N}$ terminus demonstrated that none of these residues were involved in relaxin-3 binding (Fig. 2). Rather, relaxin-3 used multiple arginine residues across a large peptide surface to interact with RXFP3, leading to a focus on potential glutamic and aspartic acid residues in the ECLs that may form electrostatic interactions with these critical arginine residues. Mutagenesis experiments demonstrated that E141 and D145 in ECL1 and E244 in ECL2 were essential for relaxin-3 binding (Fig. 2). These data were then used to dock the NMR solution structure of relaxin-3 (Rosengren et al., 2006b) into a homology model of RXFP3 based on the CXCR4 crystal structure bound to a peptide antagonist (Wu et al., 2010). The subsequent model describing the relaxin-3/RXFP3 interaction demonstrated that the three acidic residues E141, D145, and E244 likely coordinate binding to the three arginines in relaxin-3. Hence, R12 and R16 on the B-chain helical segment of relaxin-3 interact with RXFP3 residues E244 and D145, respectively. This model allows for the relaxin-3 C-terminal tail that was shown to be flexible in the NMR solution structure to insert into the classic "GPCR binding pocket" within the TM domains where relaxin-3 R26 can potentially form a salt bridge with RXFP3 E141 (Bathgate 
et al., 2013b). This model is consistent with the data showing that relaxin- 3 residues in the core $\beta$-helix are involved in RXFP3 binding, whereas the relaxin-3 C-terminal RW residues likely drive activation by interactions in the RXFP3 TM core. A slightly different relaxin-3/RXFP3 interaction model was recently described that is nonetheless still consistent with the activation mode described above (Zhang et al., 2014) (Fig. 2).

b. Transmembrane spanning regions. Chimeras were used to investigate the role of the TM spanning regions of RXFP3 and RXFP4. Replacement of TM3 or TM5 of RXFP4 with those of RXFP3 decreased affinity and abolished INSL5 activity (Zhu et al., 2008; Bathgate et al., 2013a), suggesting that these regions are necessary for INSL5 binding and activation. However, the reverse chimera of RXFP3 with TM3 and TM5 from RXFP4 had increased INSL5 binding affinity but did not activate GTP $\gamma$ S binding (Zhu et al., 2008), demonstrating that TM3 and TM5 alone are not sufficient for INSL5 activation of RXFP3. A chimera of RXFP3 with TM2, TM3, TM5, and ECL2 of RXFP4 displayed similar affinity for both relaxin-3 and INSL5 to wild-type RXFP4 (Zhu et al., 2008), suggesting that all regions influence binding. Relaxin-3 activated GTP $\gamma \mathrm{S}$ binding at this chimera similarly to wild-type RXFP4, and although INSL5 showed increased potency, it was still slightly lower than at wild-type RXFP4 (Zhu et al., 2008). This suggests that TM2, TM3, TM5, and ECL2 are all involved in ligand binding and activation of RXFP3 and RXFP4.

The mode of activation of RXFP3 and RXFP4 by their cognate ligands is more conventional than that observed for RXFP1 and RXFP2 and involves an interaction of the ligand with just the receptor $\mathrm{N}$ terminus and ECL2.

\section{Ligands That Act at Relaxin Family Peptide Receptors}

All of the relaxin family peptides that are the cognate ligands for relaxin family peptide receptors are synthesized and processed in a manner similar to insulin and have a conserved preprohormone structure (signal peptide, B-chain, C-chain, and C-terminal A-chain) that necessitates modification to produce the mature, active peptide. The signal peptide is removed first, followed by the $\mathrm{C}$ peptide, to form a mature heterodimeric peptide with two disulfide bonds between the A- and B-chains and an additional intra-A-chain bond. However, cleavage of the $\mathrm{C}$ peptide has been directly demonstrated for only some of the peptides.

Relaxin has been isolated and characterized from many species, and all show the two-chain, three disulfide-bonded structure similar to insulin (Bathgate et al., 2006a) (Fig. 3). Similar processing has been demonstrated for porcine relaxin-3 (Liu et al., 2003b) but not yet for INSL5. INSL3 has been isolated as both a prohormone form in the goat (Siqin et al., 2013) and boar (Minagawa et al., 2012) and as a processed form in the bovine (Bullesbach and Schwabe, 2002). Importantly, and as for relaxin, both processed and unprocessed forms of INSL3 are bioactive, although relaxin3 must be processed to be active at RXFP3 (Ganella et al., 2013a). Structural studies on recombinant human relaxin (Eigenbrot et al., 1991), synthetic INSL3 (Rosengren et al., 2006b), human relaxin-3 (Rosengren et al., 2006a), and INSL5 (Haugaard-Jonsson et al., 2008) have determined the three-dimensional structures of the peptides.

The crystal structure of the human relaxin peptide reveals an overall fold similar to insulin (Eigenbrot

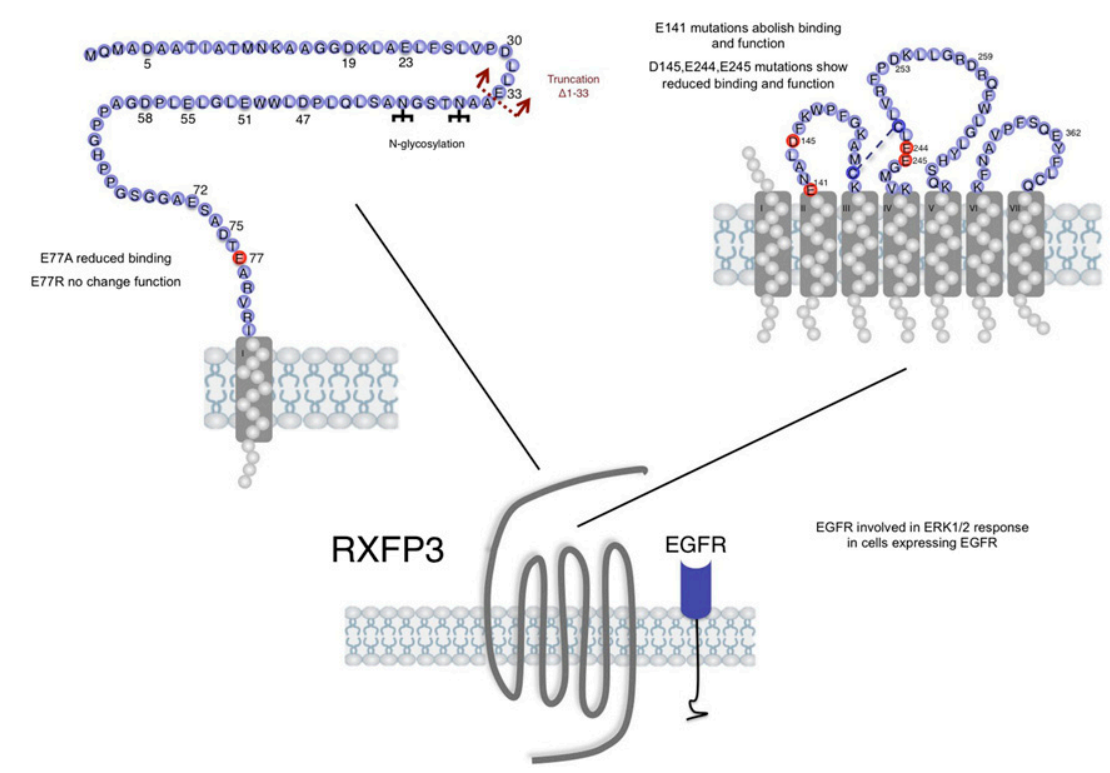

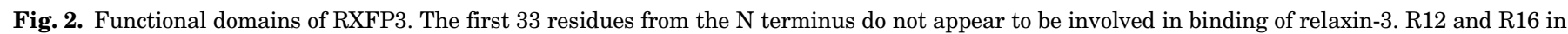

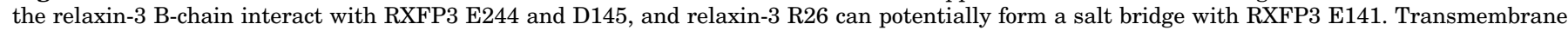

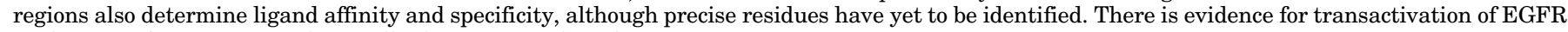
in the ERK1/2 response in those cells that express the EGFR. 
et al., 1991) as well as the formation of an asymmetric dimer, albeit in a different orientation. The main differences between relaxin family peptides occur around their termini (Rosengren et al., 2009). NMR studies of human relaxin-3 show similarities to human relaxin and insulin, although relaxin-3 has a more hydrophobic core with a condensed B-chain $\alpha$-helix, allowing interaction with W27 (Rosengren et al., 2006a). In human relaxin, the B-chain $\alpha$-helix is one turn longer, forcing the tryptophan to face away from the core of the molecule and making it solvent exposed (Rosengren et al., 2006a). NMR studies suggest that this is similar in INSL5 (Haugaard-Jonsson et al., 2009). In INSL3, the C-terminal orientation is superficially similar to that observed for human relaxin3 , with the $\mathrm{C}$ terminus contacting the hydrophobic core (Rosengren et al., 2006a). However, the $\mathrm{C}$ termini show disparity in their precise orientation that has direct ramifications for the key role of the C-terminal tryptophan in the activity of these peptides.

\section{A. Ligands That Act at Relaxin Family Peptide Receptor 1}

1. Relaxin Family Peptide Agonists. Although the relaxin peptides show considerable variation across species, alignment of amino acid sequences showed that in addition to the cysteines necessary for the two-chain structure there was a conserved RxxxRxxI/V motif in the B-chain. Examination of the X-ray crystal structure of relaxin indicates that the arginines are outward facing and located on the first and second loop of the $\alpha$-helix (Eigenbrot et al., 1991) where together with an isoleucine or valine residue they form the receptor binding site for relaxin (Schwabe and Bullesbach, 1994; Tan et al., 2002). Replacement of the arginine, isoleucine, or valine residues in this motif $(\operatorname{Rxx} \mathrm{RxxI} / \mathrm{V})$ markedly reduces or abolishes activity (Schwabe and Bullesbach, 1994; Tan et al., 2002). The relaxin A-chain shows greater sequence variation than the B-chain across species (Sherwood, 1994). Other than the conserved cysteine residues, G14 is also highly conserved and necessary for chain flexibility and structure (Bullesbach and Schwabe, 1994). Truncation of the A-chain of human relaxin produces peptides that progressively lose the ability to bind to and activate RXFP1 and RXFP2 (Hossain et al., 2008) (Fig. 3). Studies using targeted point mutations within the A-chain show an influence of the A-chain on receptor binding and activation. T16A and K17A mutations had little effect on the cAMP response to RXFP1 activation but enhanced cAMP responses after activation of RXFP2; conversely, the R22A and F23A mutations markedly reduced the activity of human relaxin at RXFP2 without reducing binding or activity at RXFP1 (Park et al., 2008); mutations S19A and L20A are also known to reduce peptide binding and activity at RXFP1 (Bullesbach and Schwabe, 1994) (Fig. 3). More recent detailed studies of the key A-chain residues (Chan et al., 2012) show that no single amino acid drives the interactions between RXFP1 and human relaxin, although Y3, L20, and F23 all have some role. These studies also identify differences in the mechanisms by which relaxin binds to and activates RXFP1 compared with RXFP2, leading to the development of a potent and selective peptide agonist for RXFP1, H2:A (4-24) (F23A) that has similar potency and biologic activity to human relaxin but no significant activity at RXFP2 (Chan et al., 2012) (Table 1). The contention that human relaxin binds to RXFP1 and RXFP2 by distinct mechanisms is further supported by the species-specific nature of these structure activity relationships: neither mouse nor rat relaxin is able to bind to or activate RXFP2 (Scott et al., 2005b), and although human relaxin-3 binds with good affinity to RXFP1, it has only a poor affinity for RXFP2 (Bathgate et al., 2006b). The importance of the peptide A-chain is also emphasized by the structure activity relationships displayed by relaxin-3. Replacement of the relaxin-3 A-chain with that of INSL5 produces a chimeric peptide (R3/I5) that no longer binds to or activates RXFP1 but has unchanged activity at RXFP3 (Liu et al., 2005a) (Fig. 3). A similar profile is attained after truncation of the relaxin-3 A-chain (Hossain et al., 2008).

Examination of the role of the B-chain in activation of RXFP1 demonstrates that it is possible to truncate both ends while still retaining binding and functional activity (Hossain et al., 2011). Peptides with a reduced B-chain [H2:(B7-24)] or reduced A- and B-chains [H2:(A4-24) (B7-24)] show some reduction in binding activity and ability to generate cAMP but have similar antifibrotic activity to human relaxin and less activity at RXFP2 (Hossain et al., 2011) (Table 1).

2. C1q-Tumor Necrosis Factor-Related Protein 8: An Additional Relaxin Family Peptide Receptor 1 Ligand? Short linear peptides derived from a naturally occurring protein containing a collagen-like repeat have been reported to act at RXFP1 (Shemesh et al., 2009). Although the effects produced by the peptides CGEN25009 and CGEN-25010 in several systems were extremely variable and the effects of human relaxin in these systems unusual (Shemesh et al., 2008, 2009), there is some evidence to suggest relaxin-like activity of these peptides in THP-1 cells and in a fibrosis model (Pini et al., 2010). In the latter study, CGEN-25009 and human relaxin increased cAMP, cGMP, and nitrite and decreased collagen deposition and increased matrix metalloproteinase-2 (MMP-2) activity in human dermal fibroblasts (Pini et al., 2010). More recent studies with these peptides and the precursor protein C1q-tumor necrosis factor-related protein 8 (CTRP8) demonstrated activation of RXFP1 (Glogowska et al., 2013) with cAMP production and a PI3K-mediated promigratory phenotype in glioblastoma cell lines and primary cells. Coimmunoprecipitation studies demonstrated a direct interaction between human CTRP8 and RXFP1. Although these studies suggest that CTRP8 or peptide fragments are able to activate RXFP1, it remains to be seen whether 


Human relaxin-2
- R13, R17 and I20 involved in
binding to RXFP1
- Truncation of B chain $\mathrm{N}$ -
terminus results in progressive
loss of binding to RXFP1 and
RXFP2. Truncation of C-
terminus past G24 and W27
results in loss of binding for
RXFP1 and RXFP2 respectively
- Minimized B(7-24) analogues
show reduced but still significant
binding affinity and activity
- R13K, R17K analogue is a
partial agonist or antagonist

B-chains

B-chains
\[ \text { A-chains } \]
Human relaxin-2
- T16A or K17A enhance cAMP
accumulation at RXFP2 only
- S19A/L20A reduce binding and
activity at RXFP1
- R22A or F23A markedly
reduced activity at RXFP2 only
- Minimized A(4-24) analogues
show increased selectivity for
RXFP1
- F23A with minimized A-chain
shows high affinity, activity and
selectivity for RXFP1

INSL3
- W27 critical for binding and
activation
- H12, R16, V19 and R20
contribute to binding
- N-terminus truncation
(PTPEMREK) affinity but no
CAMP activity - antagonist
- Cyclised modified B-chain
analogues are weak antagonists
- B-chain dimers are high affinity
antagonists

\section{Human relaxin-3}

- R8, I15, R16 and F20 involved in binding to RXFP3 and RXFP4

- R12 involved in binding to RXFP3

- R26 and W27 involved in activation but not binding to RXFP3 and RXFP4

- G23R and truncation of GSRW and $A$ chain - antagonist of RXFP3 and RXFP4

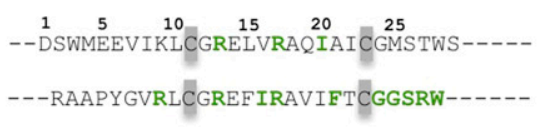

Human INSL3

Human INSL5

Human relaxin-2

Human relaxin-3

Human INSL3

Human INSL5
---PTPEMREKLCGHHFVRALVRVCGGPRWSTEA--

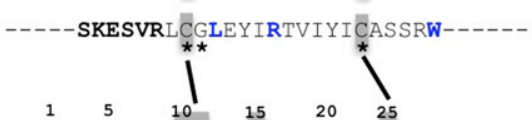

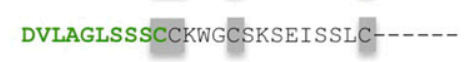

AAATNPARYCCLSGCTQQDLLTLCPY---$---Z D L Q T L C C T D G C$ CSMTDLSALC.-----

\section{Human relaxin-3}

- Truncation of $\mathrm{N}$-terminus progressive loss of binding to RXFP1 but RXFP3 binding not affected until truncation $>R 8$.

- Removal of DVLAGLSSSC agonist with high affinity and activity at RXFP3 \& RXFP4

INSL3
- C10S/C15S or DC10/DC15
affinity but no cAMP activity -
antagonists
- N-terminus truncation
(AAATNPA; AAATNPAR or
AAATNPARY) affinity but no
CAMP activity - antagonists

INSL5
- C15A and deleted ZDLQTL
easier to synthesize and partial
agonist at RXFP4
- A-chain- no activity
- Minimized analogue - partial
agonist activity
- Amidated peptide - reduced
agonist activity

\section{INSL5}

- B-chain or minimized analogues

- no activity

Fig. 3. Structure-activity relationships for the relaxin family peptides relaxin, relaxin-3, INSL3, and INSL5. All four peptides share common structural features including the two intrachain disulfide bonds and the interchain disulfide bond. However, the interaction sites between the peptides and their cognate receptors show distinct characteristics. Binding of relaxin to RXFP1 involves the RxxRxxI/V motif in the B-chain, whereas alterations in A-chain length influence activity at RXFP1 and RXFP2. Binding of INSL3 to RXFP2 involves a similar motif but displaced one turn along the $\alpha$-helix of the B-chain. $\mathrm{N}$ terminus truncation of either the A- or the B-chain reduces efficacy but not affinity and has been used as a strategy for producing antagonists. Disruption of intrachain disulfide bonds in INSL3 reduces binding to a minor extent but destroys agonist activity (C10S/C15S or C10del/C15del). Binding of relaxin-3 to RXFP3 involves predominantly the B-chain, and reduction of the A-chain removes activity at RXFP1 without affecting activity at RXFP3. Relaxin-3 R8, R16, I5, and F20 are required for binding to RXFP3 and RXFP4, with R12 required for RXFP3 but not RXFP4. Relaxin-3 R26 and W27 are required for activation of RXFP3 and RXFP4. Truncation of the B-chain and G23R produces an antagonist, and truncation of the A-chain retains high-affinity agonist activity. Binding of INSL5 to RXFP4 shows a number of differences from the relaxin-3/RXFP3 interaction. Unlike relaxin-3, the B-chain of INSL5 is inactive and minimized analogs generally show reduced affinity and efficacy. Many of the agonist and antagonist peptides active at RXFP3 are also active at RXFP4.

they are native ligands and/or whether activation of RXFP1 by CTRP8 occurs specifically in glioblastomas.

3. Small-Molecular-Weight Agonists. The recent success of the phase III clinical trial of relaxin for acute heart failure (see below) encouraged a screening campaign to identify small molecule agonists of RXFP1. By using HEK293 cells stably expressing RXFP1, 365,677 compounds were screened for their ability to cause cAMP accumulation. Both of the hits obtained contained a 2 -acetamido- $N$-phenylbenzamide structure that was used as a basis for medicinal chemistry optimization (Chen et al., 2013; http://www.ncbi.nlm.nih.gov/books/NBK153218/). Of 
TABLE 1

Ligands that interact with RXFP1

\begin{tabular}{|c|c|c|c|c|c|}
\hline Ligand & & Binding Affinity $\mathrm{p} K_{\mathrm{i}}, \mathrm{p} K_{\mathrm{D}}$ & cAMP $\mathrm{pEC}_{50}$ & $\mathrm{pERK} 1 / 2 \mathrm{pEC}_{50}$ & Reference \\
\hline Human relaxin-1 & $\mathrm{AG}$ & 8.84 & 9.1 & & Bathgate et al. (2006b) \\
\hline Relaxin & $\mathrm{AG}$ & 9.24 & 10.37 & & Hossain et al. (2008) \\
\hline Relaxin & AG & 9.75 & 9.39 & & Bathgate et al. (2013a) \\
\hline Relaxin & $\mathrm{AG}$ & & 9.71 & 9.60 & Siwek et al., unpublished \\
\hline Relaxin & $\mathrm{AG}$ & & $16.96,9.46$ & & Halls and Cooper $(2010)$ \\
\hline $\mathrm{A}(4-24)(\mathrm{B} 7-24) \mathrm{H} 2$ & AG & 6.99 & 8.22 & & Hossain et al. (2011) \\
\hline $\mathrm{A}(4-24)(\mathrm{B} 7-24) \mathrm{H} 2$ & $\mathrm{AG}$ & & 8.36 & 8.24 & Siwek et al., unpublished \\
\hline Relaxin-3 & $\mathrm{AG}$ & 7.69 & 9.36 & & Hossain et al. (2008) \\
\hline Relaxin-3 & AG & 8.6 & 8.74 & & Shabanpoor et al. (2012) \\
\hline Relaxin-3 & $\mathrm{AG}$ & & 8.85 & 8.57 & Siwek et al., unpublished \\
\hline INSL3 & $\mathrm{AG}$ & 5.68 & N.A. & & Bathgate et al. (2013a) \\
\hline $\mathrm{B}-\mathrm{R} 13 / 17 \mathrm{KH} 2$ & PA & 6.29 & 6.67 & & Hossain et al. (2010) \\
\hline $\mathrm{B}-\mathrm{R} 13 / 17 \mathrm{KH} 2$ (AT-001) & $\mathrm{PA}$ & 5.03 & 5.66 & & Silvertown et al. (2007), Neschadim et al. (2014) \\
\hline $\mathrm{B}-\mathrm{R} 13 / 17 \mathrm{KH} 2$ & PA & & 7.59 & 8.41 (7.97 ant) & Siwek et al., unpublished \\
\hline $\mathrm{A}(4-24)(\mathrm{F} 23 \mathrm{~A}) \mathrm{H} 2$ & $\mathrm{AG}$ & 9.17 & 9.82 & & Chan et al. (2012) \\
\hline Minimized relaxin-3 analog 2 & $\mathrm{AG}$ & $<5$ & N.A. & & Shabanpoor et al. (2012) \\
\hline Minimized relaxin-3 analog 3 & & $<5$ & N.D. & & Shabanpoor et al. (2012) \\
\hline ML290 & AA & N.A. & 7.03 & N.A. & Xiao et al. (2013) \\
\hline
\end{tabular}

AA, allosteric agonist; AG, agonist; BA, biased agonist; N.A., no activity; N.D., not determined; PA, partial agonist.

these compounds, a number showed reasonable potency for cAMP generation in both HEK293-RXFP1 and THP-1 cells (that endogenously express RXFP1) and had good specificity for RXFP1 versus RXFP2 (Xiao et al., 2013) (Table 1). Several of the compounds increased vascular endothelial growth factor (VEGF) gene expression in THP-1 cells with similar efficacy to relaxin, and compound 8 [ML290; 2-isopropoxy- $N$-(2-(3-(trifluoromethylsulfonyl) phenylcarbamoyl)phenyl)benzamide] was also shown to increase cellular impedance in a label-free system albeit with at least 500-fold lower potency than relaxin (Xiao et al., 2013). In addition, comparison of the binding mode of action of ML290 with that of relaxin suggests that the small-molecule agonist acts as an allosteric agonist at RXFP1 (Xiao et al., 2013; Christopoulos et al., 2014). Relaxin binds with high affinity to the LRR region of RXFP1 followed by a lower affinity interaction with ECL2 (Sudo et al., 2003; Halls et al., 2005a,b). This triggers an interaction involving the LDLa module that is essential for signaling (Scott et al., 2006; Kern et al., 2007; Kong et al., 2013). Mutations of the calcium binding asparagine within the LDLa module (D58E), produce a receptor where the cAMP response to relaxin is completely abolished but that to ML290 (compound 8) is unaffected (Xiao et al., 2013). Because, unlike the human receptor, mouse RXFP1 does not respond to ML290, chimeric constructs of human and mouse RXFP1 were used to identify the region of RXFP1 that interacts with ML290. Examination of the chimeras narrowed the region of interest down to ECL3, and two pairs of amino acids divergent between human and mouse RXFP1 were identified. The substitution of the N-terminal IL within mouse ECL3 to the VV of human RXFP1 did not rescue function (Xiao et al., 2013). C-terminal substitution of GT within human RXFP1 to the DS of mouse RXFP1 produced a chimeric human RXFP1 that did not respond to ML290. The converse substitution of DS to GT in mouse RXFP1 created a partially active receptor. The mouse receptor with a double substitution of the mouse to the human residues, IL to VV and DS to GT, produced a fully active mouse RXFP1 that responded to ML290 in a similar fashion to the human receptor (Xiao et al., 2013). The 2 -acetamido- $N$-phenylbenzamide derivatives represent the first synthetic small-molecule agonists at RXFP1. They show good potency, selectivity, and a number of relaxin-like functions in cell-based assays. They appear to behave as allosteric agonists acting at the ECL3 loop of RXFP1 (Fig. 1). It remains to be demonstrated whether the profile of activity of ML290 and similar compounds matches that of relaxin in a variety of cellular and animal disease models.

4. Relaxin Family Peptide Receptor 1 Antagonists. In contrast to the other members of the RXFP receptor family there have been few examples of peptides with antagonist properties at RXFP1. Peptide AT-001 (Neschadim et al., 2014) contains mutations of the B-chain residues R13 and R17 [that are essential for relaxin-like activity (Büllesbach et al., 1992)] to K13 and K17 (B-R13/17K relaxin). Initially, antagonism was demonstrated by lentiviral expression of the B-R13/17K relaxin, which decreased prostate cancer xenograft growth (Silvertown et al., 2007). In addition, medium from cells infected with the lentivirus showed human relaxin immunoreactivity and antagonized cAMP production from THP-1 cells in response to relaxin (Silvertown et al., 2007). However, a subsequent synthesis of the B-R13/17K peptide showed that although it competed for relaxin binding, the peptide was actually a partial agonist for cAMP production in cells overexpressing RXFP1 (Hossain et al., 2010; Neschadim et al., 2014) (Table 1). In cells expressing RXFP1 at lower (more physiologic) levels, B-R13/17K relaxin displayed functional antagonism, inhibited MCF-7 cell invasion, and prevented the inhibitory effect of relaxin on renal myofibroblast differentiation (Hossain et al., 2010). In androgen-independent PC3 prostate cancer xenografts, 
B-R13/17K suppresses growth and has antiangiogenic effects and synergizes with the chemotherapeutic agent docetaxel (Neschadim et al., 2014). The activity of B-R13/ $17 \mathrm{~K}$ at other RXFP receptors has not been examined.

The detailed understanding of the mode of interaction of peptide ligands at RXFP1 has led to the development of potent and selective agonists and also partial agonists that have antagonist properties in systems with low levels of receptor expression. An exciting recent development is the small molecular weight allosteric agonists, typified by ML290, that are currently being characterized in terms of their ability to activate the full repertoire of RXFP1 signaling.

\section{B. Ligands That Act at Relaxin Family Peptide Receptor 2}

1. Relaxin Family Peptide Agonists. INSL3 is the cognate ligand for RXFP2 that interacts with RXFP1 only at extremely high concentrations (Kumagai et al., 2002; Halls et al., 2005b), suggesting that it does not bind to or activate RXFP1 in a physiologic setting. In contrast, the cognate ligand for RXFP1, human relaxin, potently activates RXFP2, although the mode of interaction of both human relaxin and INSL3 with RXFP2 shows significant differences compared with the interaction of human relaxin with RXFP1 (see section II.B.1) (Table 2). Mutated INSL3 peptides demonstrate the utilization of distinct amino acids in the B-chain to bind to and activate RXFP2. W27 within INSL3 is critical for RXFP2 binding and activation (Bullesbach and Schwabe, 2004, 2005a), although other single amino acid replacements with either alanine or valine suggest that there are additional influences within the B-chain. Although individual substitutions only slightly reduce INSL3 binding to RXFP2, the combined mutation of H12A, R16A, V19A, R20A, and W27A abolishes binding (Rosengren et al., 2006b).

Truncation of the A-chain of human relaxin results in a peptide that progressively loses the ability to bind to and activate both RXFP1 and RXFP2 (Hossain et al., 2008), possibly because of a loss of structure in the
B-chain. However, truncation of the A-chain of INSL3 completely abolishes activation with no effect on binding (Bullesbach and Schwabe, 2005a) (Table 2). Additionally, targeted point mutations within the A-chain selectively alter the receptor binding and activation profile: the T16A and K17A mutations do not markedly alter cAMP responses to RXFP1 activation but enhance cAMP responses at RXFP2; the R22A and F23A mutations reduce human relaxin activity at RXFP2 but not at RXFP1 (Park et al., 2008). The double S19A and L20A mutations reduce peptide binding and activity at RXFP1, again suggesting a role of the A-chain in receptor binding and activation (Bullesbach and Schwabe, 1994) (Table 2). Differences in the mechanism of binding and activation between RXFP1 and RXFP2 are further supported by the species-specific nature of these structure activity relationships; thus neither mouse nor rat relaxin can bind to or activate human, rat, or mouse RXFP2 (Scott et al., 2005b), and human relaxin-3 has only a poor affinity for RXFP2 (Bathgate et al., 2006b). All of these studies highlight the importance of both the A- and B-chains for INSL3 activation of RXFP2 and show that the mechanism of action is different from the relaxin-RXFP1 interaction (Hossain et al., 2008).

2. Relaxin Family Peptide Antagonists. In contrast to RXFP1, there are numerous peptide antagonists of RXFP2 due to the difference in the mode of activation of RXFP2 by INSL3. Thus deletion of 10 residues from the $\mathrm{N}$ terminus of the INSL3 A-chain produces a peptide that still binds to RXFP2 but no longer increases cAMP accumulation (Bullesbach and Schwabe, 2005a). This truncated peptide is a specific competitive inhibitor of INSL3 at RXFP2 (Bullesbach and Schwabe, 2005a) (Table 2). In addition, deletion of eight $\mathrm{N}$-terminal residues of the B-chain produces an INSL3 peptide that retains binding affinity but loses agonist activity (Bullesbach and Schwabe, 2005a). Substitution of eight A- or B-chain residues with alanine does not affect receptor signaling (Bullesbach and Schwabe, 2005a). Disruption of the intra-A-chain disulfide bond (C10S/C15S; or $\mathrm{C} 10 \mathrm{del} / \mathrm{C} 15 \mathrm{del})$ produces peptides that retain RXFP2

TABLE 2

Ligands acting at RXFP2

\begin{tabular}{|c|c|c|c|c|}
\hline Ligand & & Binding Affinity $\mathrm{p} K_{\mathrm{i}}, \mathrm{p} K_{\mathrm{D}}$ & $\mathrm{cAMP}_{\mathrm{pEC}} \mathrm{p}_{50}$ & Reference \\
\hline Relaxin & $\mathrm{AG}$ & 8.48 & 9.13 & Hossain et al. (2008) \\
\hline Relaxin & $\mathrm{AG}$ & 8.96 & 7.88 & Bathgate et al. (2013a) \\
\hline INSL3 & $\mathrm{AG}$ & 9.34 & 10.35 & Hossain et al. (2008) \\
\hline INSL3 & $\mathrm{AG}$ & 9.71 & 8.27 & Bathgate et al. (2013a) \\
\hline $\mathrm{A}(4-24)(\mathrm{B} 7-24) \mathrm{H} 2$ & PA & $>6$ & $>6$ & Hossain et al. (2011) \\
\hline $\mathrm{A}(4-24)(\mathrm{F} 23 \mathrm{~A}) \mathrm{H} 2$ & $\mathrm{AG}$ & $<6$ & $<6$ & Chan et al. (2012) \\
\hline $\mathrm{A}(9-26)$ INSL3 & ANT & 9.14 & N.A. & Bullesbach and Schwabe (2005a); Hossain et al. (2008) \\
\hline $\mathrm{A}(10-24)$ INSL3 & ANT & 8.67 & N.A. & Hossain et al. (2008) \\
\hline $\mathrm{A}(\mathrm{C} 10 / 15 \mathrm{~S}) \mathrm{INSL} 3$ & ANT & 8.59 & N.A. & Zhang et al. (2010) \\
\hline $\mathrm{A}(\Delta 10 / 15 \mathrm{C}) \mathrm{INSL} 3$ & ANT & 8.32 & N.A. & Zhang et al. (2010) \\
\hline Cyclic INSL3 B-chain analog 6 & ANT & 6.65 & N.A. & Shabanpoor et al. (2007) \\
\hline Dimeric INSL3 B-chain analog 8 & ANT & 8.50 & N.A. & Shabanpoor et al. (2011) \\
\hline ML290 & AA & & N.A. & Xiao et al. (2013) \\
\hline
\end{tabular}

AA, allosteric agonist; AG, agonist; ANT, antagonist; N.A., no activity; PA, partial agonist. 
binding but do not activate cAMP signaling (Zhang et al., 2010) (Table 2). The INSL3 B-chain alone is also a RXFP2 antagonist, although it only has low affinity for the receptor (Del Borgo et al., 2006; Shabanpoor et al., 2007). Modifications of the single-chain structure result in modest gains in affinity, but larger gains are obtained by linking two B-chains using the native cysteine residues (Shabanpoor et al., 2010) (Table 2). Modifications of this B-chain dimer resulted in antagonists with affinities only slightly lower than INSL3 (Shabanpoor et al., 2011).

Despite the similarity between RXFP1 and RXFP2 and their cognate ligands relaxin and INSL3, the precise mode of peptide activation of the receptors is quite different. This has facilitated the successful development of a number of antagonists for RXFP2.

\section{Ligands That Act at Relaxin Family Peptide Receptor 3}

1. Relaxin Family Peptide Agonists. Human relaxin-3 has a tertiary structure, determined by solution NMR spectroscopy, that is similar to insulin and other relaxin family peptides (Rosengren et al., 2006a). However, unlike the interaction of human relaxin with RXFP1 that requires both A- and B-chains for binding and activation, the human relaxin-3 B-chain alone can bind to and activate both RXFP3 and RXFP4 (Liu et al., 2003b). Replacement of the human relaxin-3 A-chain with the INSL5 A-chain (R3/I5) does not influence RXFP3 binding or activation but markedly reduces the ability of the peptide to bind to and activate RXFP1 (Liu et al., 2005a) (Table 3). Similarly, truncation of the human relaxin-3 A-chain reduces binding to and activation of RXFP1 but retains full activity at RXFP3 (Hossain et al., 2008). The additional deletion of the A-chain disulfide bond results in the complete loss of RXFP1 binding and activation with little effect on RXFP3 binding and activation (Shabanpoor et al., 2012). Further development of this analog with a B23-27 deletion and addition of an arginine at B23 led to a high-affinity, RXFP3selective, competitive antagonist (analog 3) (Table 3).

Site-directed mutagenesis of B-chain residues reveals that R8, R16, I5, and F20 are important for human relaxin-3 binding to RXFP3 and RXFP4 (Kuei et al., 2007), with R12 also required for binding to RXFP3 but not RXFP4 (Kuei et al., 2007). R26 and W27 toward the $\mathrm{C}$ terminus of the B-chain are required for activation of RXFP3 (Kuei et al., 2007). Truncation of the $\mathrm{C}$ terminus of the B-chain to $\mathrm{C} 22$ and the serendipitous addition of an additional arginine residue at the $\mathrm{N}$ terminus in the recombinant production system, when combined with the A-chain of INSL5, led to the discovery of a highaffinity RXFP3-selective antagonist R3(B $\Delta 23-27) \mathrm{R} / \mathrm{I} 5$ (Kuei et al., 2007) (see section II.C.4; Table 3). A singlechain antagonist has since been developed based on the $\mathrm{B}$-chain of R3(B $\Delta 23-27) \mathrm{R} / \mathrm{I} 5$ with the cysteine residues mutated to serine (H3 B1-22R) (Haugaard-Kedstrom et al., 2011) (Table 3).
2. Biased Agonists Acting at Relaxin Family Peptide Receptor 3. Ligand-directed signaling bias is increasingly common in GPCR pharmacology and describes stabilization of distinct receptor confirmations by ligands, resulting in selective activation of downstream signal transduction pathways (Baker and Hill, 2007; Evans et al., 2010; Kenakin and Miller, 2010). Although the original studies suggested that only relaxin-3 and its B-chain bound to and activated RXFP3, it is now clear that several relaxin peptides interact with RXFP3 to activate distinct signaling profiles through different, although sometimes overlapping pathways (Fig. 5). Initial studies showed that human relaxin-3 was selective for RXFP3 in both binding and AC inhibition assays, with no receptor activation by human relaxin or INSL3 (Liu et al., 2003b). However, cross-reactivity with other relaxin peptides was not examined over a wider range of signal transduction pathways, and the sensitivity of inhibitory cAMP assays can be influenced by the degree of activation of AC by forskolin and the time of stimulation (for detailed description see section III.C.2).

3. Allosteric Modulators. A novel selective allosteric modulator of RXFP3 has been described (Alvarez-Jaimes et al., 2012). 135PAM1 (3-[3,5-bis(trifluoromethyl)phenyl]1-(3,4-dichlorobenzyl)-1-[2-(5-methoxy-1H-indol-3-yl)ethyl] urea) is a positive allosteric modulator (PAM) that curiously only displays activity with C-terminal amidated relaxin-3 or R3/I5 (probe selectivity). Binding studies conducted in HEK293-RXFP3 cell membranes showed that 135PAM1 does not compete with $\left[{ }^{125} \mathrm{I}\right] \mathrm{R} 3 / \mathrm{I} 5$ (amide) binding at up to $1 \mu \mathrm{M}$ but at higher concentrations enhances binding consistent with activity as a PAM. The R3/I5(amide) peptide itself competes for [ $\left.{ }^{125} \mathrm{I}\right] \mathrm{R} 3 / \mathrm{I} 5$ (amide) binding in a conventional manner (AlvarezJaimes et al., 2012). In HEK293-RXFP3 cells coexpressing G $\alpha$ qi5, 135PAM1 increased calcium responses to $\mathrm{EC}_{20}$ concentrations of relaxin-3(amide) or R3/I5(amide) but not the free-acid $(\mathrm{OH})$ peptides. Concentration-response curves to the amidated but not the free-acid form of the peptides were shifted in a limiting manner by 135PAM1. Similar specificity of 135PAM1 was also displayed in a reporter gene assay that measured inhibition of CRE activity in cells expressing RXFP3 (Table 3 ). This is the only published example to date of a small molecule compound with activity at RXFP3. The discovery of 135PAM1 identifies an allosteric site on RXFP3 that can be modulated by small molecules.

4. Relaxin Family Peptide Receptor 3 Antagonists. The synthetic peptide R3(B $\Delta 23-27) R / I 5$ that was discovered as a RXFP3 antagonist has been instrumental in defining the physiologic functions of the receptor. Specificity for RXFP3 was initially achieved by synthesizing a chimeric peptide featuring the A-chain of INSL5 in combination with the B-chain of relaxin-3. The RXFP3 agonist properties of this peptide chimera were then markedly reduced by truncation of the relaxin-3 B-chain (Kuei et al., 2007; Hossain et al., 2009). Serendipitously, 
TABLE 3

Ligands acting at RXFP3

\begin{tabular}{|c|c|c|c|c|c|c|c|}
\hline Ligand & & $\begin{array}{l}\text { Binding } \\
\text { Affinity } \\
\mathrm{p} K_{\mathrm{i}}, \mathrm{p} K_{\mathrm{D}}\end{array}$ & $\begin{array}{l}\mathrm{cAMP} \\
\mathrm{pEC}_{50}\end{array}$ & $\begin{array}{l}\mathrm{GTP}_{\mathrm{pEC}} \mathrm{S} \\
\mathrm{pE}_{50}\end{array}$ & $\underset{\mathrm{pEC}}{\mathrm{pERK} 1 / 2}$ & $\begin{array}{l}\text { P38 MAPK } \\
\text { pEC }_{50}\end{array}$ & Reference \\
\hline Relaxin-3 & AG & 8.48 & 8.26 & & & & Hossain et al. (2008) \\
\hline Relaxin-3 & $\mathrm{AG}$ & 7.78 & 9.0 & & & & Shabanpoor et al. (2012) \\
\hline Relaxin-3 & $\mathrm{AG}$ & 9.29 & & 9.48 & & & Zhu et al. (2008) \\
\hline Relaxin-3 & $\mathrm{AG}$ & 8.92 & 9.17 & & 9.23 & & van der Westhuizen et al. (2007) \\
\hline Relaxin-3 & AG & & 9.13 & & 9.18 & & van der Westhuizen et al. (2010) \\
\hline Relaxin-3 & $\mathrm{AG}$ & & & & 9.93 & 8.9 & Kocan et al. (2014) \\
\hline R3/I5 & $\mathrm{AG}$ & 9.28 & 9.35 & & & & Liu et al. (2005a) \\
\hline R3/I5 & AG & 9.31 & 9.33 & & & & Kuei et al. (2007) \\
\hline Relaxin & BA & 10.0 & 7.05 & & 7.99 & & van der Westhuizen et al. (2010) \\
\hline Relaxin & $\mathrm{BA}, \mathrm{PA}$ & & & & 7.15 & 8.35 & Kocan et al. (2014) \\
\hline INSL5 & ANT & 7.01 & & N.A. & & & Zhu et al. (2008) \\
\hline $\begin{array}{l}\text { Minimized relaxin-3 } \\
\quad \text { analog } 2\end{array}$ & $\mathrm{AG}$ & 7.87 & 8.43 & & 10.4 & & Shabanpoor et al. (2012) \\
\hline $\begin{array}{l}\text { Minimized relaxin-3 } \\
\quad \text { analog } 3\end{array}$ & ANT & 7.6 & N.A. & & & & Shabanpoor et al. (2012) \\
\hline Relaxin-3 B-chain dimer & $\mathrm{AG}$ & 6.61 & & & & & van der Westhuizen et al. (2007) \\
\hline $\mathrm{R} 3(\mathrm{~B} \Delta 23-27) \mathrm{R} / \mathrm{I} 5$ & ANT & 8.49 & N.A. $\sim 8$ & & & & Haugaard-Kedstrom et al. (2011) \\
\hline $\mathrm{R} 3(\mathrm{~B} \Delta 23-27) \mathrm{R} / \mathrm{I} 5$ & ANT & 9.17 & N.A. & & & & Kuei et al. (2007) \\
\hline $\mathrm{R} 3(\mathrm{~B} \Delta 23-27) \mathrm{R} / \mathrm{I} 5$ & $\begin{array}{l}\text { ANT, PA, } \\
\text { BA }\end{array}$ & & & & 8.87 & 9.02 & Kocan et al. (2014) \\
\hline R3 B1-22R & ANT & 7.44 & N.A. & & & & Haugaard-Kedstrom et al. (2011) \\
\hline 135PAM1 & PAM & & 6.12 & & & & Alvarez-Jaimes et al. (2012) \\
\hline Relaxin-3 B-chain & AG & & 7.06 & $\sim 7.0$ & & & $\begin{array}{l}\text { Liu et al. }(2003 b) \\
\text { Liu et al. }(2005 a)\end{array}$ \\
\hline
\end{tabular}

AA, allosteric agonist; AG, agonist; ANT, antagonist; BA, biased agonist; N.A., no activity; PA, partial agonist

upon recombinant production of $\mathrm{R} 3(\mathrm{~B} \Delta 23-27) / \mathrm{I} 5$ an extra arginine remained at the $\mathrm{N}$ terminus due to incomplete peptidase "trimming of the RR from the RRRR furin cleavage domain" to produce the peptide R3(B $\Delta 23-27) \mathrm{R} / \mathrm{I} 5$. This peptide binds to RXFP3 and RXFP4 with high affinity and is a potent antagonist at RXFP3 in both rats and in human in vitro systems. Synthetic R3(B $\Delta 23-27) / I 5$ (lacking R23) has lower affinity at RXFP3 and has weak agonist properties (Hossain et al., 2009), highlighting that the extra R23 creates an additional interaction with RXFP3 (Table 3). Administration of R3(B $\Delta 23-27) \mathrm{R} / \mathrm{I} 5$ intracerebroventricularly to rats blocks food intake stimulated by the RXFP3 agonist R3/I5. It recently became apparent that R3(B $\Delta 23-27) \mathrm{R} / \mathrm{I} 5$ has a complex antagonist profile and blocks some but not all pathways activated by RXFP3. It also has weak biased agonist properties (see section III.C.2). In addition, studies have revealed that the intra-A-chain disulfide bond in relaxin-3 is not important for interaction with RXFP3 but is necessary for the interaction of relaxin-3 with RXFP1. Analogs without this A-chain disulfide bond together with truncation of 10 amino acids from the $\mathrm{N}$ terminus of the A-chain remain potent RXFP3 agonists. If in addition, GGSRW is removed from the B-chain and replaced with $R$, the peptide becomes a high-affinity antagonist with similar biologic activity to R3(B $\Delta 23-27) R / I 5$ (Shabanpoor et al., 2012) (Table 3).

Studies with R3(B $\Delta 23-27) R / 15$ suggested that the additional RXFP3 affinity provided by the R23 residue might result in the B-chain only R3(B $\Delta 23-27) \mathrm{R}$ having significant affinity for RXFP3 (Table 3 ). This has been proved experimentally and $\mathrm{H} 3 \mathrm{~B} 1-22 \mathrm{R}$ is a high-affinity antagonist of RXFP3 that is far easier to produce than the two-chain peptides (Haugaard-Kedstrom et al., 2011). Importantly this peptide is also specific for RXFP3 and has no activity at RXFP4. The peptide was shown to have efficacy in vivo and blocks increases in feeding produced by intracerebroventricular injection of R3/I5 in rats (Haugaard-Kedstrom et al., 2011). It was subsequently used to demonstrate a role for the endogenous relaxin-3 system in addiction in rats (Ryan et al., 2013b) and in motivated food seeking and consumption in mice (Smith et al., 2014).

The structure-activity relationships for the interaction of relaxin peptides with RXFP3 are now well understood. Unlike RXFP1 and RXFP2, RXFP3 can be activated by peptides comprising only the B-chain, and a number of selective agonists and antagonists have been developed. Both relaxin and some RXFP3 antagonists display biased agonist properties at RXFP3. However, the utility of a small molecule allosteric modulator in vivo is currently limited by probe selectivity.

\section{Ligands That Act at Relaxin Family Peptide Receptor 4}

1. Relaxin Family Peptide Agonists. INSL5 is the cognate ligand for RXFP4 (Liu et al., 2005b). INSL5 has many of the characteristics of an incretin being secreted from enteroendocrine $L$ cells and regulating insulin secretion and glucose homeostasis (Burnicka-Turek et al., 2012). Synthesis of human INSL5 represents considerable challenges so that some of the characterization of the peptide has been performed with mouse INSL5 that has $71 \%$ homology (Belgi et al., 2011). Compared with human INSL5, mouse INSL5 displays an 8-fold increase in affinity in binding assays and a 5-fold greater potency in 
cAMP inhibition assays, possibly related to the overall greater positive charge associated with the nonconserved residues (Belgi et al., 2011) (Table 4). In addition, the amidated peptides show a considerable reduction in potency compared with the free acids (Belgi et al., 2011). Although relaxin-3 binds both RXFP3 and RXFP4 with high affinity, INSL5 is actually a weak antagonist at RXFP3 (Zhu et al., 2008) (Table 4). Furthermore, although the relaxin-3 B-chain alone was sufficient to bind and activate RXFP3, the INSL5 A- and B-chains alone are inactive at RXFP4 (Belgi et al., 2013).

The relaxin-3 B-chain alone is also a weak agonist at RXFP4 (Liu et al., 2003a, 2005a) (Table 4). Up to seven residues of the $\mathrm{N}$ terminus of the $\mathrm{B}$-chain of relaxin-3 can be deleted with little effect on binding or activation of RXFP4 (Kuei et al., 2007; Hossain and Wade, 2010), whereas the $\alpha$-helical region of the B-chain is important for activity at RXFP4 (Hossain and Wade, 2010). R12 and R16 are important for binding to both RXFP1 and RXFP3, whereas only R16 is important for RXFP4 binding (Kuei et al., 2007). A critical residue for RXFP4 and RXFP3 binding appears to be F20, and mutations of this residue are associated with a marked loss of affinity (Kuei et al., 2007; Hossain and Wade, 2010). In addition, the $\mathrm{C}$ terminus of the relaxin-3 B-chain appears to be important for activation of RXFP4, and mutation of these residues blocks agonist activity without influencing affinity (Hossain and Wade, 2010).

2. Modified Peptide Agonists. The potential of RXFP4 as a therapeutic target and the difficulty involved in the synthesis of human INSL5 has led to the search for more easily synthesized and selective analogs. Minimized analogs of relaxin-3 display some interesting properties. Removal of the first eight residues of the A-chain of relaxin-3 produces an analog (analog 2) with similar binding affinity and potency to relaxin-3 at RXFP3 and RXFP4 but no activity at RXFP1 (Shabanpoor et al., 2012) (Table 4). This activity is retained in a peptide where the B-chain is truncated by nine residues (Shabanpoor et al., 2012). These studies indicate that the $\mathrm{C}$ terminus of the $\mathrm{B}$-chain and the interchain disulfide bonds are the major structural features required for activity at RXFP4.

3. Relaxin Family Peptide Receptor 4 Antagonists. Several of the compounds developed as antagonists of RXFP3 also have antagonist properties at RXFP4. These peptides include the minimized relaxin-3 analog 3 (minimized A-chain and truncated B-chain) that has no agonist activity at RXFP4 but only a slightly reduced binding affinity (Shabanpoor et al., 2012) (Table 4). Likewise the R3(B $\Delta 23-27) R / I 5$ RXFP3 antagonist also is a high-affinity antagonist at RXFP4 (Kuei et al., 2007). Interestingly, the single-chain variant of this peptide $\mathrm{H} 3$ B1-22R does not bind to RXFP4 (Haugaard-Kedstrom et al., 2011) (Table 4).

Much less information is available on RXFP4 compared with the other family members, but this has been accelerating due to the identification of INSL5 as a likely incretin. There is now a good understanding of the mode of interaction of INSL5 with RXFP4 and an increasing range of synthetic peptide agonists and antagonists have been developed.

\section{Signal Transduction Pathways}

\section{A. Relaxin Family Peptide Receptor 1 Signaling}

1. Canonical Signaling Pathways. RXFP1 displays pleiotropic signaling to a variety of pathways including cAMP accumulation, activation of MAP kinases, tyrosine kinases, and NO as well as pathways associated with connective tissue metabolism (Fig. 4). Early studies before receptor identification, showed that treatment with relaxin increased cAMP accumulation in THP-1 cells (Parsell et al., 1996), MCF-7 cells (Bigazzi et al., 1992), the mouse pubic symphysis (Braddon, 1978), uterine strips (Sanborn et al.,

TABLE 4

Ligands acting at RXFP4

\begin{tabular}{|c|c|c|c|c|c|}
\hline Ligand & & Binding Affinity $\mathrm{p} K_{\mathrm{i}}, \mathrm{p} K_{\mathrm{D}}$ & $\mathrm{cAMP}_{\mathrm{pEC}} \mathrm{p}_{50}$ & $\mathrm{GTP}_{\gamma \mathrm{S}} \mathrm{pEC}_{50}$ & Reference \\
\hline hINSL5 & AG & 7.33 & 8.51 & & Shabanpoor et al. (2012) \\
\hline hINSL5 & $\mathrm{AG}$ & 7.59 & 8.62 & & Belgi et al. (2013) \\
\hline hINSL5 & $\mathrm{AG}$ & 8.66 & & 8.94 & Zhu et al. (2008) \\
\hline hINSL5 & $\mathrm{AG}$ & 8.8 & 8.92 & 8.89 & Liu et al. (2005b) \\
\hline hINSL5 amide & $\mathrm{AG}$ & 6.94 & 7.48 & & Belgi et al. (2011) \\
\hline mINSL5 & AG & 8.47 & 9.29 & & Belgi et al. (2013) \\
\hline mINSL5 amide & $\mathrm{AG}$ & 7.07 & 6.85 & & Belgi et al. (2011) \\
\hline Relaxin-3 & $\mathrm{AG}$ & 8.81 & & 9.01 & Zhu et al. (2008) \\
\hline Relaxin-3 & $\mathrm{AG}$ & 8.83 & 9.02 & & Kuei et al. (2007) \\
\hline Relaxin-3 B-chain & $\mathrm{AG}$ & 7.07 & 7.06 & $\sim 7.0$ & Liu et al. (2003b) \\
\hline Relaxin-3 B-chain & $\mathrm{AG}$ & & 6.9 & & Liu et al. (2005a) \\
\hline $\mathrm{R} 3 / \mathrm{I} 5$ & $\mathrm{AG}$ & 8.92 & 9.04 & & Liu et al. (2005a) \\
\hline R3/I5 & AG & 8.88 & 8.94 & & Kuei et al. (2007) \\
\hline Minimized hINSL5 analog 7 & $\mathrm{PA}$ & 6.28 & 7.40 & & Belgi et al. (2013) \\
\hline Relaxin-3 & $\mathrm{AG}$ & & 8.94 & & Shabanpoor et al. (2012) \\
\hline Minimized relaxin-3 analog 2 & AG & 7.1 & 7.7 & & Shabanpoor et al. (2012) \\
\hline Minimized relaxin-3 analog 3 & ANT & 6.6 & N.A. & & Shabanpoor et al. (2012) \\
\hline $\mathrm{R} 3(\mathrm{~B} \Delta 23-27) \mathrm{R} / \mathrm{I} 5$ & ANT & $\sim 8$ & N.D. & & Haugaard-Kedstrom et al. (2011) \\
\hline $\mathrm{R} 3(\mathrm{~B} \Delta 23-27) \mathrm{R} / \mathrm{I} 5$ & ANT & 8.64 & N.A. & & Kuei et al. (2007) \\
\hline R3 B1-22R & & N.A. & N.D. & & Haugaard-Kedstrom et al. (2011) \\
\hline
\end{tabular}

AG, agonist; ANT, antagonist; N.A., no activity; PA, partial agonist. 
Basal
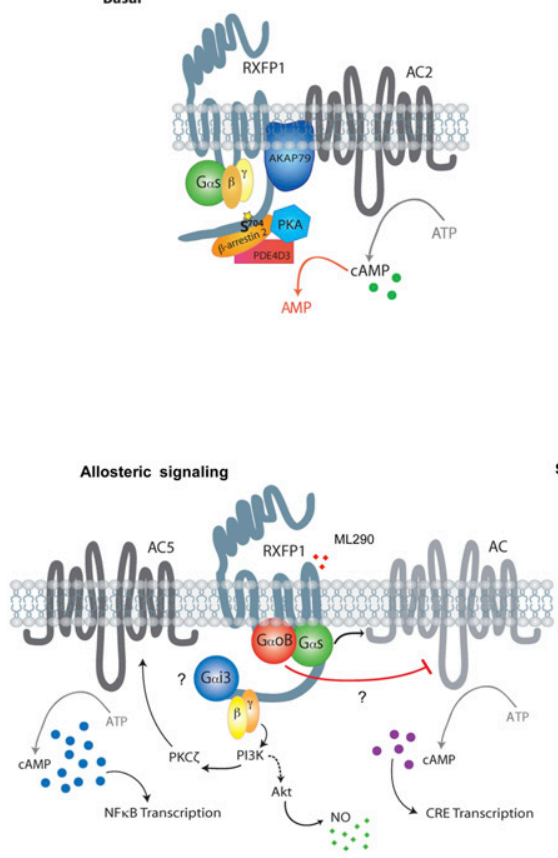
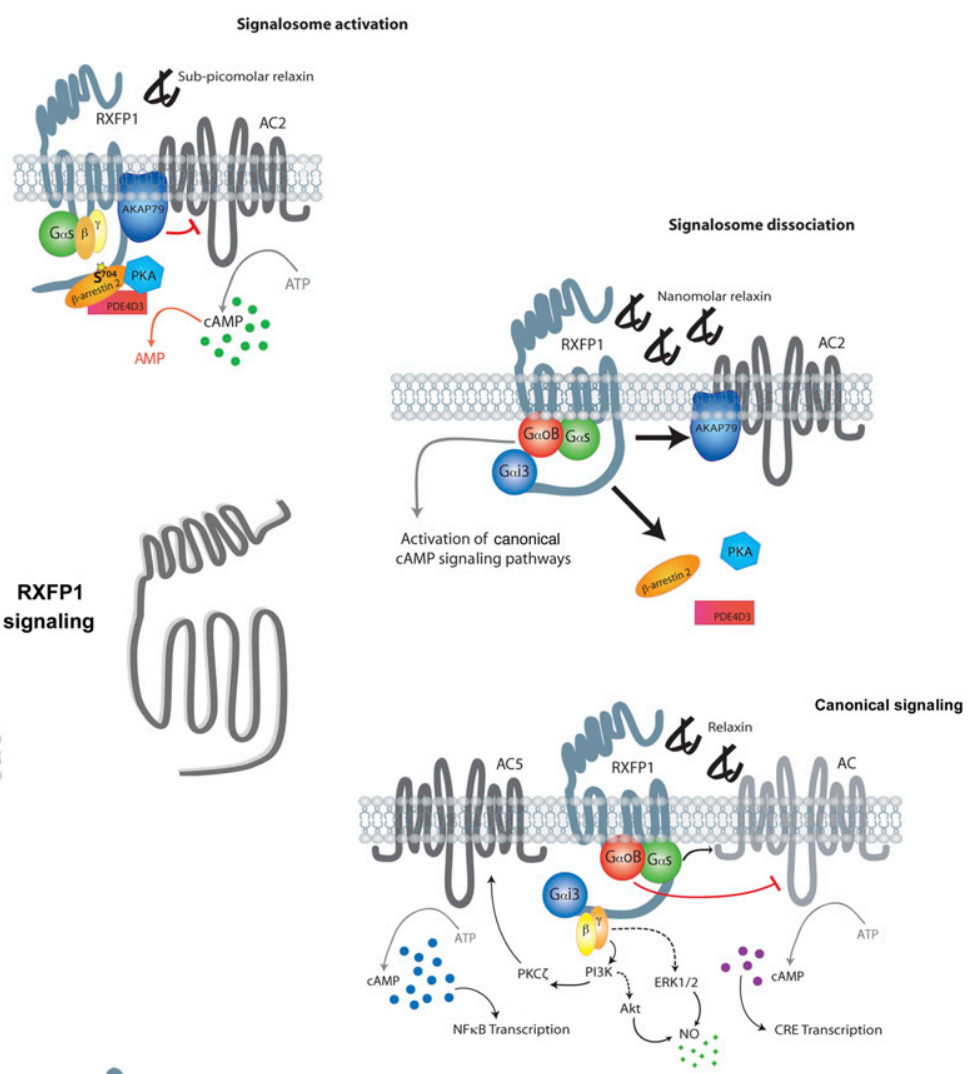

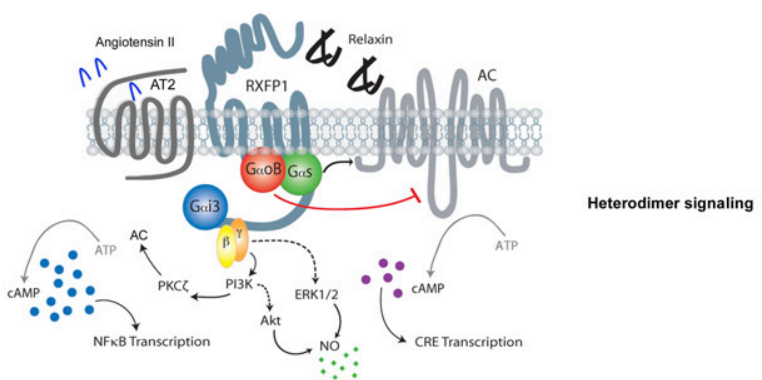

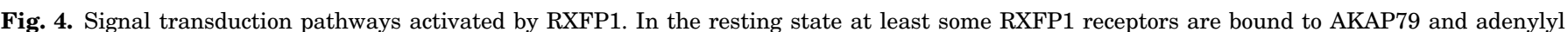

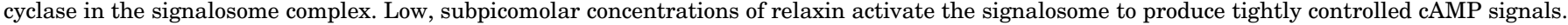

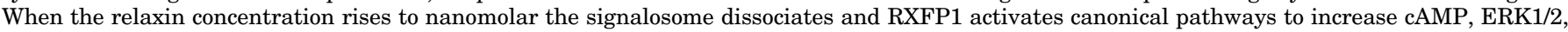

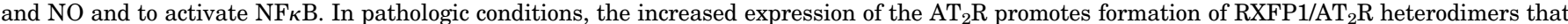

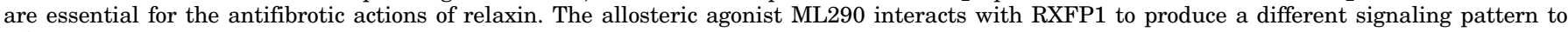
relaxin.

1980), uterine longitudinal muscle (Osa et al., 1991) from estrogen-primed rats, and in cultures of human endometrial cells (Fei et al., 1990), human endometrial glandular epithelial cells (Chen et al., 1988), newborn rhesus monkey uterine cells (Kramer et al., 1990), rat myometrial cells (Hsu et al., 1985), and rat anterior pituitary cells (Cronin et al., 1987). The importance of cAMP as a signaling pathway for relaxin was confirmed on RXFP1 deorphanization, because constitutively active mutants of RXFP1 (TM6: D637Y) increased cAMP accumulation in a ligandindependent manner (Hsu et al., 2000, 2002). RXFP1 couples to $\mathrm{G} \alpha_{\mathrm{s}}$ to increase cAMP (Hsu et al., 2000, 2002; Halls et al., 2006), an effect that is negatively modulated by coupling to $\mathrm{G} \alpha_{\mathrm{oB}}$ (Halls et al., 2006) (Fig. 4). RXFP1 also couples to $\mathrm{G} \alpha_{\mathrm{i} 3}$ to activate a delayed surge of cAMP accumulation via a $\mathrm{G} \beta \gamma$-PI3K-PKC $\zeta$ pathway that activates
AC5 (Nguyen et al., 2003; Nguyen and Dessauer, 2005b; Halls et al., 2006, 2009b). The delayed pathway was initially identified in THP-1 cells that endogenously express RXFP1 where relaxin causes a biphasic increase in cAMP accumulation, with the later phase partially blocked by the PI3K inhibitors LY294002 [2-(4-morpholinyl)8-phenyl-1(4H)-benzopyran-4-one hydrochloride] and Wortmannin. This was confirmed when relaxin stimulation of RXFP1 was also shown to increase PI3K activity (Nguyen et al., 2003). PKC $\zeta$ was proposed as the candidate protein that linked PI3K activation to cAMP formation and is an atypical isoform that is insensitive to diacylglycerol and calcium but activated by phosphatidylinositol 3,4,5-trisphosphate and other lipids (Ono et al., 1989; Nakanishi et al., 1993; Standaert et al., 2001). Treatment with porcine relaxin causes 
a concentration-dependent translocation of $\mathrm{PKC} \zeta$ to the cell membrane in MCF-7 (human breast cancer), PHM1-31 (pregnant human myometrial), MMC (mouse mesangial), and THP-1 cells (Nguyen and Dessauer, 2005b), with translocation dependent upon PI3K activation by relaxin but independent of cAMP accumulation. cAMP increases mediated by relaxin were also dependent upon PKC $\zeta$ expression (Nguyen and Dessauer, 2005b). Although in MCF-7 cells, relaxin stimulation activates $\mathrm{PI} 3 \mathrm{~K}$ and causes translocation of $\mathrm{PKC} \zeta$ without increasing cAMP accumulation, transfection of AC5, but not AC2 or AC4, into these cells produces a cAMP response to relaxin (Nguyen and Dessauer, 2005a). Thus RXFP1 activates $\mathrm{PI} 3 \mathrm{~K}$ and causes translocation of $\mathrm{PKC} \zeta$ to the cell membrane, which in turn stimulates AC5 (Fig. 4).

This pathway also occurs in HEK293 cells transiently or stably expressing RXFP1 (Halls et al., 2006, $2009 \mathrm{~b}$ ) and is downstream of $\mathrm{G} \alpha$ and $\mathrm{G} \beta \gamma$ subunits, because transfection of cells with $\mathrm{G} \alpha_{\mathrm{i} / \mathrm{o}}$ mutants that are insensitive to $\mathrm{ADP}$ ribosylation by pertussis toxin (PTX) show coupling of RXFP1 to $\mathrm{G} \alpha_{\mathrm{i} 3}$ (in addition to $\mathrm{G} \alpha_{\mathrm{S}}$ and inhibitory $\mathrm{G} \alpha_{\mathrm{oB}}$ ) as the mediator of the $\mathrm{G} \beta \gamma$ PI3K-PKC $\zeta$-AC5 pathway. This also occurs in endogenous settings: in rat left atria, relaxin increases cAMP accumulation that is reduced by PTX pretreatment, which also reduced the inotropic and chronotropic responses to relaxin (Kompa et al., 2002); in the failing human heart there is increased $\mathrm{G} \alpha_{\mathrm{i} / \mathrm{o}}$ expression (Eschenhagen et al., 1992; Bohm et al., 1994), and the positive inotropic effects of relaxin in humans are preserved in this condition (Dschietzig et al., 2011).

The final 10 amino acids of the RXFP1 $\mathrm{C}$ terminus, particularly R752, are essential for activation of the $\mathrm{G} \alpha_{\mathrm{i} 3}$ pathway (Halls et al., 2009b) (Fig. 1), as is the presence of lipid-rich membrane domains, suggesting compartmentalization of the RXFP1-stimulated cAMP response (Halls et al., 2009b). GTP $\gamma$ S-immunoprecipitation studies show that $\mathrm{G} \alpha_{\mathrm{i} 3}$ is activated immediately after RXFP1 stimulation, suggesting that the delay observed is downstream of the $\mathrm{G}$ protein and probably involves the translocation of PKC $\zeta$ (Halls et al., 2009b). In HEK293 cells, only activation of $\mathrm{G} \alpha_{\mathrm{s}^{-}}$and $\mathrm{G} \alpha_{\mathrm{oB}}$-dependent cAMP signaling pathways increases CRE-mediated gene transcription, whereas $\mathrm{G} \alpha_{\mathrm{i} 3}$-mediated signaling appears to selectively regulate nuclear factor- $\kappa \mathrm{B}(\mathrm{NF} \kappa \mathrm{B})$-dependent gene transcription (Halls et al., 2007a), again suggesting that there is compartmentalization of signaling events and inferring that distinct physiologic outcomes can be anticipated downstream of different cAMP signaling branches (Halls et al., 2007a). cAMP accumulation may also occur in response to relaxin by a $\mathrm{G}$ protein-independent mechanism, and in some cells may be downstream of a tyrosine kinase. In THP-1 cells and cultures of primary human myometrial or endometrial stromal cells, porcine relaxin increased cAMP accumulation that was blocked by inhibition of tyrosine kinase activity (Kuznetsova et al., 1999; Bartsch et al., 2001; Anand-Ivell et al., 2007; Heng et al., 2008). This response could be potentiated by the phosphotyrosine phosphatase inhibitors $[\mathrm{bpV}$ (phen) and $\mathrm{mpV}($ pic)] that mimic tyrosine kinase activation (Bartsch et al., 2001). In human lower uterine segment fibroblasts, relaxin stimulation caused tyrosine phosphorylation of cellular extracts, with no effect upon cAMP accumulation (Palejwala et al., 1998). The tyrosine kinase-dependent increase in cAMP accumulation may occur by inhibition of a PDE, thereby preventing cAMP hydrolysis and thus reflexively increasing cAMP levels. However, the same tyrosine kinase inhibitors do not affect relaxin-stimulated cAMP accumulation in HEK293 cells expressing RXFP1 (Anand-Ivell et al., 2007), emphasizing the variation in cellular responses between different cell types. In cells using the tyrosine kinase pathway, there was some degree of cAMP inhibition by the PI3K inhibitor LY294002 (Anand-Ivell et al., 2007; Heng et al., 2008) and evidence of a negative feedback loop involving PKA (Anand-Ivell et al., 2007).

The stimulation of NO production, cGMP generation, and PKG activation provides another crucial pathway by which relaxin exerts its effects. Depending on the cell type under investigation, relaxin may activate endothelial nitric oxide synthase (eNOS) (Baccari et al., 2007; Dschietzig et al., 2012) and neuronal NOS (nNOS) (Baccari et al., 2004; Mookerjee et al., 2009) or stimulate the expression of inducible NOS (iNOS) (Bani et al., 1998a; Alexiou et al., 2013) (Fig. 4). In rat isolated lungs, the relaxin-mediated iNOS upregulation depends on a subtle balance between stimulatory ERK1/2 activation and counterregulatory PI3K stimulation (Alexiou et al., 2013).

In addition to cAMP accumulation, many cells that express RXFP1 such as human endometrial stromal cells (Zhang et al., 2002), THP-1 cells and primary cultures of human coronary artery cells, pulmonary artery smooth muscle cells, renal myofibroblasts (Mookerjee et al., 2009), and fibrochondrocytes (Ahmad et al., 2012) respond to relaxin with a rapid activation of ERK1/2 (Fig. 4). In normal human endometrial cells relaxin causes rapid but transient phosphorylation of ERK1/2, with a peak response between 5 and 10 minutes (Zhang et al., 2002). The same time course was observed for phosphorylation of MEK and CREB, but relaxin treatment did not affect Akt or JNK phosphorylation and treatment with a MEK inhibitor blocked phosphorylation of ERK1/2 in response to relaxin, suggesting that MEK is activated upstream of ERK1/2 (Zhang et al., 2002). Increased phosphorylation of ERK1/2 (pERK1/2) in response to relaxin is also observed in THP-1 cells and cultures of human coronary artery and pulmonary artery smooth muscle cells and is associated with increased transcription of VEGF (Zhang et al., 2002).

In HeLa cells and primary human umbilical vein endothelial cells, the pERK1/2 response is more prolonged (Dschietzig et al., 2003). In HeLa, EAhy926 (an endothelial cell line), HT-29 (a colonic cell line), and in primary 
fibrochondrocyte cells, relaxin increased the phosphorylation of both ERK1/2 and Akt after 30 minutes (Dschietzig et al., 2009a; Ahmad et al., 2012). In primary fibrochondrocytes, treatment with relaxin also activates PI3K, $\mathrm{PKC} \zeta, \mathrm{NF} \kappa \mathrm{B}$, c-fos, and Elk-1, all of which influence the expression of MMP-9 (Ahmad et al., 2012). Relaxin also produces a sustained increase in pERK1/2 in rat renal myofibroblasts, potentiated by inhibition of $\mathrm{G} \alpha_{\mathrm{i} / \mathrm{o}}$ by PTX, suggesting that phosphorylation of ERK1/2 may be downstream of $\mathrm{G}$ protein coupling (Mookerjee et al., 2009). In contrast, in human vascular smooth muscle cells, relaxin stimulation did not affect $\mathrm{pERK} 1 / 2$ levels but instead increased the phosphorylation of p38 mitogenactivated protein kinase (MAPK) (Dschietzig et al., 2003). In rat pulmonary arterial endothelial and smooth muscle cells, relaxin increased iNOS activity and expression dependent on a balance of $\mathrm{pERK} 1 / 2$ and PI3K pathway activation (Alexiou et al., 2013). Thus, although relaxin increases phosphorylation of a number of kinases in multiple cell types, the precise isoform and mode of activation appears to vary, and the physiologic consequences of activation of these pathways are as yet unclear.

Several of the aforementioned canonical pathways are involved in the well established antifibrotic actions of relaxin. Transforming growth factor (TGF)- $\beta_{1}$ is profibrotic, causing Smad2/3 phosphorylation, translocation of the Smad complex to the nucleus, and activation of profibrotic genes. Inhibition of this process by relaxin involves activation of PI3K and NOS-NO-cGMP signaling (Ahmad et al., 2012; Chow et al., 2012). There is also recent evidence that suggests that relaxin influences differentiation of neonatal fibroblasts into myofibroblasts by preventing the inhibition of Notch-1 signaling by TGF$\beta_{1}$. Notch-1 signaling controls cell differentiation and fate and is involved in fibrosis (Fan et al., 2011). The Notch-1 pathway comprises Notch-1 receptors that, when activated by the TM ligands Jagged1/2, release the Notch intracellular domain that migrates to the nucleus to inhibit transcription (Chillakuri et al., 2012). Downregulation of Notch-1 is a necessary step in the differentiation of rat cardiac fibroblasts into myofibroblasts and is induced by TGF- $\beta_{1}$ (Fan et al., 2011). A recent study demonstrates that relaxin prevents the enhancement by TGF- $\beta_{1}$ of cytoskeletal assembly in 3T3 cells and primary neonatal fibroblasts as measured by $\mathrm{F}$-actin and vinculin staining (Sassoli et al., 2013). In addition, relaxin prevented the TGF- $\beta_{1}-$ mediated increases in $\alpha$-SMA, type 1 collagen, and tissue inhibitor of metalloproteinase (TIMP)-2, while preventing the TGF- $\beta_{1}$-mediated decreases in MMP-2 and MMP-9. Relaxin treatment also prevented the downregulation of Notch- 1 expression by TGF- $\beta_{1}$ as well as the inhibition of Notch intracellular domain and Jagged-1 release (Sassoli et al., 2013).

2. Noncanonical Signaling Pathways. Relaxin activates the GR, a nuclear receptor that acts as a liganddependent transcription factor (Dschietzig et al., 2004).
The activation, and the subsequent changes in gene transcription, may account for the many effects of relaxin upon the expression levels of a variety of proteins, including those involved in connective tissue metabolism (see above). Relaxin treatment of THP-1 cells that have differentiated into a macrophage phenotype blunts the stimulated production of cytokines including interleukin (IL)-1, IL-6, and tumor necrosis factor (TNF)- $\alpha$, and this effect is abolished by the GR antagonists RU-486 [mifepristone; $17 \beta$-hydroxy-11 $\beta$-(4-dimethylamino-phenyl)$17 \alpha$-(1-propinyl)estra-4,9-dien-3-on] and D06 [bis(4$N, N$-dimethylaminophenyl)(2-chloro-5-nitrophenyl) methane] (Dschietzig et al., 2004, 2009a). Relaxin coimmunoprecipitates with the GR, and the amount of the GR within the nucleus increases after 30-minute stimulation with relaxin. In whole cell and in fluorescence polarization assays, relaxin displaces classic glucocorticoids from the GR (Dschietzig et al., 2004, 2009a). The region of relaxin that binds to the GR differs from that involved in relaxin binding to RXFP1, because a modified relaxin that was unable to activate RXFP1 could still interact with the GR, and relaxin was found to cause phosphorylation of S211 of the GR, which is used as a biomarker of agonistrelated receptor activation (Dschietzig et al., 2009a). Indeed relaxin, via its interaction with the GR, is also able to autoregulate its own expression by binding to half-sites of glucocorticoid response elements located 160-200 bp upstream of transcription start at the human RLN2 promoter (Dschietzig et al., 2009b). Actions at the GR are also involved in the vasodilator effects of relaxin. In rat aortic rings, relaxation responses to acetylcholine are impaired by TNF- $\alpha$ in a model of endothelial dysfunction, and the effect is reversed by relaxin (Dschietzig et al., 2012). The reversal of the effect of TNF- $\alpha$ by relaxin is blocked by the PI3K inhibitor Wortmannin but also by the GR and progesterone receptor antagonist RU-486. In rat primary aortic endothelial cells TNF- $\alpha$ treatment increased endothelin-1 (ET-1) and arginase II expression, decreased superoxide dismutase (SOD)-1 expression, and stimulated superoxide and nitrotyrosine formation (Dschietzig et al., 2012). All of these effects were restored or attenuated by relaxin treatment acting at the GR (Dschietzig et al., 2012). The findings suggest that relaxin has a protective effect on endothelial dysfunction mediated not only by actions on the canonical PI3K-Akt-eNOS pathway but also by its actions at the GR, with SOD-1 upregulation being dependent on relaxin-GR-c/EBP- $\beta$ signaling (Dschietzig et al., 2012). Reports that appear to confirm the principal relaxin-GR finding include relaxin-related protection in a rodent model of severe acute pancreatitis that is markedly attenuated after cotreatment with RU-486 (Cosen-Binker et al., 2006), relaxin-induced GR activation in reporter gene experiments (Halls et al., 2007a), and blockade of relaxin-mediated decreases in granulocyte macrophage colony-stimulating factor and IL-8 secretion from primary decidual macrophages by RU-486 (Horton et al., 2011). 
Hitherto, the precise mode of interaction between relaxin and GR has been unclear and there are contradictory findings regarding RXFP1 involvement in the relaxin-GR pathway. Although relaxin seems to interact with GR in spleen cells that do not express RXFP1 (Dschietzig et al., 2004) and also after chemical modification to destroy RXFP1 affinity (Dschietzig et al., 2009a), RXFP1 was necessary for relaxin to activate the GR in an in vitro study using reporter genes (Halls et al., 2007a).

3. The Relaxin Family Peptide Receptor 1 SignalosomeA Protein Complex That Facilitates High-Sensitivity Signaling in Response to Relaxin. Responses to relaxin produced by activation of canonical signaling pathways occur in the nanomolar concentration range (Bathgate et al., 2013a). However, circulating concentrations of relaxin are usually lower than those required to produce cellular responses by canonical mechanisms (Sherwood, 2004). Recently, a constitutive RXFP1-dependent cAMP response has been identified using Förster resonance energy transferbased cAMP biosensors in single rat cardiac fibroblasts, HeLa cells, and HEK293 cells expressing RXFP1 (Halls and Cooper, 2010). The response is dependent upon a protein complex, or signalosome, linked to the relaxin receptor, and the signalosome is highly sensitive to attomolar concentrations of relaxin. Importantly, this may provide the basis for some of the physiologic responses to relaxin when it is present in the circulation at levels below those necessary to activate canonical signaling pathways. The signalosome consists of RXFP1 that is scaffolded to AC2 by AKAP79, facilitating efficient activation of the $\mathrm{AC}$ by $\mathrm{G} \alpha_{\mathrm{s}}$ and $\mathrm{G} \beta \gamma$ subunits (Fig. 4). The levels of cAMP produced are tightly regulated by the activity of PKA-activated PDE4D3 that itself is scaffolded to the receptor $\mathrm{C}$ terminus (specifically requiring S704) by $\beta$-arrestin-2 (Halls and Cooper, 2010). The signalosome possesses stimulatory (AKAP79 and AC2) and regulatory ( $\beta$-arrestin-2, PKA, and PDE4D3) arms that are both spatially and functionally distinct. Knockdown of AKAP79 has no effect on the regulatory arm, and knockdown of $\beta$-arrestin- 2 does not influence the stimulatory arm of the signalosome. In addition, there is no effect of inhibitors of classic pathway specific proteins (including $\mathrm{G} \alpha_{\mathrm{i} / \mathrm{o}}, \mathrm{PI} 3 \mathrm{~K}$, and PKC) on cAMP generated in response to subpicomolar concentrations of relaxin and no effect of inhibition of signalosome-specific proteins (including AC2, AKAP79, and $\beta$-arrestin-2) upon classic relaxin cAMP signaling. The pathways also generate cAMP in quite distinct regions of the cell. Thus signalosome-specific AC2 is known to be preferentially excluded from lipid-rich domains (Willoughby et al., 2007), whereas activation of the $\mathrm{G} \alpha_{\mathrm{i} 3}$ pathway with nanomolar relaxin concentrations depends upon lipidrich domains in HEK293 cells (Halls et al., 2009b). Because AC2 expression occurs predominantly in brain, lung, skeletal muscle, heart, and uterine myometrium (Defer et al., 2000; Willoughby et al., 2007; Sadana and Dessauer, 2009), it is likely that some tissues display
RXFP1 signalosome signaling, whereas others do not, which may help to determine the physiologic role of signalosome-localized RXFP1.

Targeted protein knockdown or overexpression of dominant negative mutants (Halls and Cooper, 2010) suggests that the regulatory arm is isoform-specific (i.e., interacts only with PDE4D3) and that assembly of the components depends upon constitutive association between the receptor and $\beta$-arrestin-2 (not $\beta$-arrestin-1) (Halls and Cooper, 2010). The regulatory arm also has some unusual features that call into question some of the current paradigms surrounding $\beta$-arrestins and receptor desensitization and internalization (DeFea, 2011). For example, the $\beta_{2}$-adrenoceptor is phosphorylated by $\mathrm{G}$ protein receptor kinase (GRK)-2 after receptor activation, and subsequently becomes a substrate for $\beta$-arrestin- 2 that triggers formation of clathrin-coated vesicles and internalization (for review, see Luttrell and Gesty-Palmer, 2010). However, and in contrast, the interaction between RXFP1 and $\beta$-arrestin- 2 is constitutive and does not involve receptor activation or phosphorylation and does not appear to be involved in desensitization (Halls and Cooper, 2010). Although the interaction involves S704 of RXFP1 (Halls and Cooper, 2010), there is no significant increase in RXFP1 phosphorylation after stimulation with high concentrations of relaxin nor any effect of receptor activation on desensitization or internalization (Tan et al., 1998; Callander et al., 2009). Thus $\beta$-arrestin- 2 appears to act solely as a scaffold for the formation of the regulatory complex and has no role in receptor desensitization or internalization. On the other hand, the stimulatory arm of the signalosome depends upon a specific and constitutive association between helix 8 of the receptor and AKAP79 (Fig. 4). However, AKAP79 is also known to scaffold many other proteins including other GPCRs, PKC, several ion channels, and a number of AC isoforms (see Baillie et al., 2005; Dessauer, 2009; Skroblin et al., 2010; Halls, 2012). The activity of $\mathrm{AC}$ isoforms may be variably influenced by interaction with AKAP79 and AC2 activity is inhibited (Efendiev et al., 2010), although this appears to be primarily offset in RXFP1 signalosomes by the scaffolding of RXFP1 and AC2. Signalosome signaling occurs quite separately from canonical signaling, and the signalosome complex dissociates when exposed to nanomolar concentrations of relaxin (Halls and Cooper, 2010).

High-sensitivity signaling such as that exhibited by the RXFP1 signalosome has been previously shown for suppression of proinflammatory cytokine production by IL-15 (Alleva et al., 1997), proliferation of helper T cells by IL-1 (Orencole and Dinarello, 1989), the effects of neuropeptides and neurosteroids in nociception (SanchezBlazquez and Garzon, 1995; Ueda et al., 2001), and the long-term effects of TGF- $\beta$ on basal follicle-stimulating hormone levels (Ying et al., 1986). Thus the RXFP1signalosome represents a highly sensitive signaling platform that produces tightly regulated cAMP responses over a wide range of subpicomolar relaxin concentrations. 
Although this represents a mechanism whereby cells and tissues could respond to low circulating levels of relaxin, a precise physiologic role for the mechanism has yet to be determined.

4. Homo- and Hetero-Oligomerization of RXFP1 and Its Functional Consequences. Like many other class A GPCRs, there is evidence from bioluminescence resonance energy transfer (BRET) studies that RXFP1 receptors form homo- and heterodimers (Kern et al., 2008; Svendsen et al., 2008a,b) in the absence of, and independent of, ligand occupation of the receptor (Svendsen et al., 2008a,b). It has been suggested that dimer formation is necessary for signal transduction, with ligand binding occurring at the LRR region of one dimer partner, followed by interaction of the bound ligand with the ECL2 of the second partner and initiation of signaling (Kong et al., 2010). However, at present there are no experiments with two inactive mutant receptor dimer partners that show complementation and rescue of function by dimerization to support this concept. One functional consequence of dimerization is negative cooperativity (Svendsen et al., 2008a,b), where the two binding sites do not have a fixed affinity and the affinity of each remaining unoccupied receptor binding site decreases as occupancy increases. The evidence for negative cooperativity at RXFP1 comprises receptor kinetic studies where the rate of dissociation after incubation of cells expressing RXFP1 with $\left[{ }^{125} \mathrm{~T}\right]$ human relaxin was determined after equilibration followed by infinite dilution in the absence or presence of unlabeled ligand. The presence of unlabeled ligand was associated with modest increases in the rate of dissociation, suggesting negative cooperativity. It should be borne in mind, however, that such behavior does not necessarily reflect the formation of dimers (Chabre et al., 2009), and studies are required using receptors that retain function but are unable to form dimers or on receptors expressed in model phospholipid bilayers that allow examination of their functional characteristics when in monomeric form (Whorton et al., 2007; Velez-Ruiz and Sunahara, 2011). Nevertheless, there are two intriguing, functionally relevant consequences of negative cooperativity: an increased functional range of the ligand over a wider concentration range and a decrease in ligand residence time at the receptor as the free ligand concentration increases, potentially allowing selective activation of different signaling pathways (Shymko et al., 1997). The negative cooperativity concentration-response curve for relaxin binding to RXFP1 is linear, although the absence of a protein structure precludes conclusions regarding the functional consequences of this observation (Svendsen et al., 2008b).

Receptor oligomerization has been suggested to explain one intriguing aspect of relaxin-RXFP1 pharmacology, the phenomenon of bell-shaped concentration-response curves that are observed in many bioassays from studies in recombinant and primary cell systems (Halls et al., 2006; Sarwar et al., 2015), animal studies (Danielson and Conrad, 2003; Debrah et al., 2005), and clinical trials
(Teerlink et al., 2009). Negative cooperativity could be a potential explanation, but currently available information does not appear to support this mechanism. Although both RXFP1 and RXFP2 display negative cooperativity, bell-shaped curves for cAMP accumulation are observed only in response to 3- or 30-minute activation of RXFP1 or 30-minute activation of RXFP2, but not 3-minute activation of RXFP2 (Halls et al., 2006). It could be argued that the RXFP2 system at 3 minutes is not in equilibrium and that the effect of increasing receptor occupation balances out the reduced receptor affinity, but this does not convincingly explain the difference between RXFP1 and RXFP2 at the same time point. In studies of human vascular cells that endogenously express RXFP1, venous endothelial and smooth muscle cells displayed pronounced bell-shaped concentration-response curves, yet arterial smooth muscle cells showed conventional sigmoidal concentration-response curves (Sarwar et al., 2015). This would appear to be incompatible with an explanation based on RXFP1 homodimer formation and negative cooperativity.

There is also evidence for dimer formation between the haloreceptor and a number of splice variants (encoding the LDLa module only and up to eight LRR) and dimers are present at all stages of receptor translocation from the endoplasmic reticulum to the plasma membrane (Kern et al., 2008). This suggests an important role of dimerization over the lifetime of the receptor. Dimers were also formed between the haloreceptor and a TM-only domain receptor, although the $\mathrm{BRET}_{2}$ ratio was decreased compared with that obtained for haloreceptor dimers (Svendsen et al., 2008a,b). This suggests that although the TM domain is sufficient for dimerization, the ectodomains play an important role in stabilizing the oligomer. Examination of three of the splice variants cloned from human fetal membranes showed that although their expression in HEK293 cells produced no response to relaxin (Kern et al., 2008), coexpression with RXFP1 produced a parallel shift to the right of the cAMP concentration-response curve. Because the splice variants also markedly reduced cell surface expression of RXFP1, the reduced responses are likely due to a dominant negative effect of the splice variants (Kern et al., 2008).

Recent studies also suggest that there is heterodimer formation between RXFP1 and other GPCRs. The antifibrotic actions of relaxin are believed to be mediated through a RXFP1-pERK1/2-nNOS-NO-cGMP dependent pathway, leading to regulation of collagen-degrading MMPs (Chow et al., 2012). The effect of relaxin is completely absent in angiotensin type 2 receptor $\left(\mathrm{AT}_{2} \mathrm{R}\right)^{-/ \mathrm{y}}$ mice or in mice treated with the $\mathrm{AT}_{2} \mathrm{R}$ antagonist PD123319 [S-(+)-1-[(4-(dimethylamino)-3-methylphenyl) methyl]-5-(diphenylacetyl)-4,5,6,7-tetrahydro- $1 H$ imidazo[4,5-c]pyridine-6-carboxylic acid di(trifluoroacetate)], suggesting that the $\mathrm{AT}_{2} \mathrm{R}$ is necessary for the antifibrotic actions of relaxin. Interestingly BRET studies show that 
RXFP1 and $\mathrm{AT}_{2} \mathrm{R}$ form constitutive heterodimers, indicating that the receptor complex is responsible for the novel pharmacology observed (Fig. 4). There is no direct binding of relaxin to the $\mathrm{AT}_{2} \mathrm{R}$, and the peptide does not affect the BRET signal from RXFP1-AT ${ }_{2} \mathrm{R}$ complexes (Chow et al., 2014). Importantly, the findings also explain why the antifibrotic actions of relaxin are only observed in pathologic states: under normal physiologic conditions $\mathrm{AT}_{2} \mathrm{R}$ are expressed at low levels but are dramatically increased with injury and disease (Siragy and Carey, 1997; Matsubara, 1998; Carey, 2005; Savoia et al., 2006; Jones et al., 2008).

It is interesting to note that the expression of relaxinRXFP1 as measured by immunohistochemistry and expression of the renin-angiotensin-aldosterone system may both be increased during pregnancy (Ferreira et al., 2009; Cheung and Lafayette, 2013), suggesting another state where RXFP1- $\mathrm{AT}_{2} \mathrm{R}$ heterodimers may have a functional role. However, a study in rat primary mesangial cells did not corroborate this suggestion (Carvalho et al., 2012). Although RXFP1 and $\mathrm{AT}_{2} \mathrm{R}$ were increased in cells obtained from pregnant rats compared with virgin animals and the hyporesponsiveness of cells from pregnant animals to angiotensin II could be attributed to increased endogenous relaxin production, the $\mathrm{AT}_{2} \mathrm{R}$ antagonist PD123319 had no effect. Thus, the issue of RXFP1- $\mathrm{AT}_{2} \mathrm{R}$ interaction during pregnancy remains a hypothesis.

5. Allosteric Agonist Signaling at Relaxin Family Peptide Receptor 1. The small molecular weight ligand ML290 (see section II.A.3) is an allosteric agonist at RXFP1 (Xiao et al., 2013) that unlike relaxin does not require the LDLa module for signaling (Xiao et al., 2013). ML290 interacts with ECL3 of RXFP1, specifically requiring G659 and T660 within ECL3 for activity (Xiao et al., 2013) (Fig. 1). ML290 does not interact with the classic binding sites on RXFP1 used by relaxin and does not compete for ${ }^{125}$ I relaxin binding (Table 1 ).

\section{B. Relaxin Family Peptide Receptor 2 Signaling}

1. Canonical Signaling Pathways. INSL3 is the cognate ligand at RXFP2, although human relaxin but not rat or mouse relaxin, also activates the receptor. Despite the similar structure of relaxin and INSL3 and their receptors RXFP1 and RXFP2, the mode of interaction of these ligands with RXFP2 is different (see section I.B.1). Signaling pathways that are initiated after stimulation of RXFP2 are simpler than those observed for RXFP1. INSL3 (or relaxin) stimulation of HEK293-RXFP2 cells causes coupling to $\mathrm{G} \alpha_{\mathrm{s}}$ to increase cAMP accumulation and to $\mathrm{G} \alpha_{\mathrm{oB}}$ to negatively modulate this effect (Kumagai et al., 2002; Halls et al., 2006). This stage of signaling therefore closely resembles the first stage of RXFP1 signaling in response to relaxin (Halls et al., 2006). The contribution of $\mathrm{G} \alpha_{\mathrm{oB}}$ to the response is seen by the increase in cAMP after removal of $\mathrm{G} \beta \gamma$ using $\beta$ ARK-ct or inhibition of $\mathrm{G} \alpha_{\mathrm{i} / \mathrm{o}}$ with PTX (Halls et al., 2006).
Interestingly, for RXFP2 there is no evidence for constitutive activity or a high-sensitivity response to INSL3 as seen with the RXFP1 signalosome (Halls and Cooper, 2010); neither is there activation of the $\mathrm{G} \alpha \alpha_{\mathrm{i} 3}$-cAMP signaling pathway that is unique to RXFP1 (Halls et al., 2006, 2009b). Activation of RXFP2 (by either relaxin or INSL3) also induces increased CRE-dependent gene transcription (Halls et al., 2007a) as observed with relaxin acting at RXFP1.

$\mathrm{G} \alpha_{\mathrm{S}}$ and $\mathrm{G} \alpha_{\mathrm{oB}}$ are also involved in cAMP accumulation in cells that endogenously express RXFP2. In rat gubernacular cells (Kumagai et al., 2002), in a human osteoblast cell line (MG-63) (Ferlin et al., 2008), and in mouse primary Leydig cells (Pathirana et al., 2012), INSL3 stimulation of RXFP2 leads to increased cAMP accumulation, probably involving $\mathrm{G} \alpha_{\mathrm{s}}$. However, and in contrast, primary cultures of testicular germ cells and oocytes respond to INSL3 activation of RXFP2 with a PTX-sensitive inhibition of cAMP accumulation (Kawamura et al., 2004), consistent with RXFP2 coupling to $\mathrm{G} \alpha_{\mathrm{oB}}$. Thus, as for RXFP1, the net signaling outcome of RXFP2 stimulation will depend upon the signaling components (especially $\mathrm{G}$ protein isoforms) that are expressed in a particular cell type.

In response to relaxin, RXFP1 activates a number of signaling pathways including cAMP, cGMP, and pERK1/2. Relaxin also activates the GR to alter gene expression. RXFP1 forms protein complexes or signalosomes that facilitate high-sensitivity responses to relaxin. In contrast, INSL3 activates RXFP2 to increase or decrease cAMP in a cell-dependent manner. INSL3 does not activate the GR and RXFP2 does not form signalosomes.

\section{Relaxin Family Peptide Receptor 3 Signaling}

1. Canonical Signaling Pathways. Activation of RXFP3 by human relaxin-3 causes PTX-sensitive inhibition of forskolin-stimulated cAMP accumulation, suggesting that the receptor is coupled to inhibitory $\mathrm{G} \alpha_{\mathrm{i} / \mathrm{o}}$ proteins (Liu et al., 2003b). Activation of $\mathrm{G} \alpha_{\mathrm{i} / \mathrm{o}}$ proteins by RXFP3 is also associated with PI3K- and PKC-dependent phosphorylation of ERK1/2 and other MAPKs (van der Westhuizen et al., 2007, 2010) (Fig. 5). Both human relaxin-3 or its B-chain compete for $\left[{ }^{125} \mathrm{I}\right]$ human relaxin-3 binding and inhibit AC activity (Liu et al., 2003b), but more recent studies show that human relaxin also interacts with human RXFP3 to activate a unique signaling pattern (see below) (van der Westhuizen et al., 2010).

Forskolin-stimulated cAMP accumulation in CHOK1 or HEK293 cells stably expressing RXFP3 or in mouse SN56 cells that endogenously express the receptor (Liu et al., 2003b; van der Westhuizen et al., 2007) is inhibited after stimulation with human relaxin-3, and this response is completely prevented by pretreatment with PTX. In CHO-K1 cells transiently transfected with PTX insensitive (C351I mutation) variants of $\mathrm{G} \alpha_{\mathrm{i} / \mathrm{o}}$ proteins and treated with PTX (to remove the influence of endogenous $\mathrm{G} \alpha_{\mathrm{i} / \mathrm{o}}$ proteins), $\mathrm{G} \alpha_{\mathrm{i} 2}$ was the major $\mathrm{G}$ protein 
involved in the inhibition of cAMP accumulation, whereas in HEK293 cells, $\mathrm{G} \alpha_{\mathrm{i} 3}, \mathrm{G} \alpha_{\mathrm{oB}}$, and $\mathrm{G} \alpha_{\mathrm{oA}}$ were all involved (van der Westhuizen, 2008). Thus, the G proteins involved, although broadly similar, can vary with the different cell types. Similar effects are observed with human relaxin-3 B-chain peptides or with human relaxin and porcine relaxin in the CHO-K1, HEK293, and SN56 cell backgrounds (Liu et al., 2003b; van der Westhuizen et al., 2007, 2010).

Human relaxin-3 causes a rapid and transient increase in ERK1/2 phosphorylation (peak response 2-5 minutes) in CHO-K1 and HEK293 cells stably expressing human RXFP3 (CHO-RXFP3 or HEK-RXFP3, respectively; van der Westhuizen et al., 2007, 2010) (Fig. 5). The human relaxin-3 B-chain dimer also activates ERK1/2, albeit with low potency and efficacy (van der Westhuizen et al., 2007), and there is also a weak response to human relaxin but not to porcine relaxin or INSL3 (van der Westhuizen et al., 2010) (Fig. 5). PTX pretreatment caused an approximately $90 \%$ inhibition of human relaxin-3 stimulated ERK1/2 phosphorylation in CHORXFP3 cells, whereas the signal was completely abolished in HEK-RXFP3 or SN56 cells (van der Westhuizen et al., 2007). Thus coupling of RXFP3 to PTX-sensitive $\mathrm{G} \alpha_{\mathrm{i} / \mathrm{o}}$ proteins mediates ERK1/2 phosphorylation in both recombinant and endogenous systems (van der Westhuizen et al., 2007). There are two pathways downstream of $\mathrm{G} \alpha_{\mathrm{i} / \mathrm{o}}$ involved in RXFP3-mediated ERK1/2 phosphorylation in CHO-K1, HEK293, and SN56 cells. About $50 \%$ of the MAPK response is blocked by the PI3K inhibitors LY294002 or Wortmannin, whereas the remainder is inhibited by general and isoform-selective PKC inhibitors (van der Westhuizen et al., 2007). The ERK1/2 response appears to be involved in central feeding responses in rats (Morikawa et al., 2004; Shen et al., 2004; Sasaguri et al., 2005), suggesting that it is physiologically relevant.

Signaling pathway analysis using reporter genes gives a broader view of the signal transduction mechanisms activated by RXFP3. Many MAPK signaling pathways [p38 MAPK (Roux and Blenis, 2004); JNK (Davis, 2000); and ERK1/2 (Price et al., 1996; Whitmarsh and Davis, 1996)] converge on AP-1 elements to increase gene transcription, therefore AP-1-linked reporter genes together with selective inhibitors provide useful information on signaling in different cellular backgrounds. Inhibition of $\mathrm{G} \alpha_{\mathrm{i} / \mathrm{o}}$ proteins after pretreatment with PTX blocks RXFP3-mediated AP-1 reporter activation in SN56 cells but not in CHO-RXFP3 and HEK-RXFP3 cells (van der Westhuizen et al., 2010), suggesting that AP-1 reporter gene activation was downstream of $\mathrm{G} \alpha_{\mathrm{i} / \mathrm{o}}$ in the mousederived cell line but not in the other two cell types (van der Westhuizen et al., 2010). Human relaxin-3mediated AP-1 reporter gene activation in CHO-RXFP3 and SN56 cells is completely blocked by the p38 MAPK inhibitor (RWJ67657; 4-[4-(4-fluorophenyl)-1-(3-phenylpropyl)-5-(4-pyridinyl)-1H-imidazol-2-yl]-3-butyn-1-ol), whereas MEK (PD98059; 2'-amino-3'-methoxyflavone) or JNK inhibition (SP600125; anthra[1,9-cd]pyrazol-6 (2H)-one) only partially blocks AP-1 activation (as shown by a decrease in relaxin-3 potency). In contrast in HEKRXFP3 cells, JNK inhibition completely blocks human relaxin-3-stimulated AP-1 reporter activation, whereas p38 MAPK or MEK inhibition partially blocked AP-1 activation (van der Westhuizen et al., 2010). This suggests that although all three MAPKs are involved in human relaxin-3-mediated AP-1 activation, the hierarchy of the different signaling pathways varies with the cell background. Several studies show that MAPK signaling is activated in forced swim tests in rats, where there are dramatic increases in pMEK1/2, pERK1/2, and pJNK1/2/ 3 (Shen et al., 2004). Although this is associated with an increase in relaxin-3 mRNA in the nucleus incertus (NI) (Tanaka et al., 2005), direct links between human relaxin-3, RXFP3, MAPK phosphorylation, and stress responses remain to be demonstrated in brain.

In another reporter gene assay, in CHO-K1 and HEK293 cells transiently expressing human RXFP3 and in SN56 cells endogenously expressing mouse RXFP3, activation of RXFP3 by human relaxin-3 increased $\mathrm{NF}_{\kappa} \mathrm{B}$ reporter gene activation (van der Westhuizen et al., 2010). Activation of $\mathrm{NF} \kappa \mathrm{B}$ was blocked by PTX pretreatment (van der Westhuizen et al., 2010), suggesting that the response occurs downstream of $\mathrm{G} \alpha_{\mathrm{i} / \mathrm{o}}$. The physiologic significance of this pathway remains to be determined.

2. Ligand-Directed Signaling Bias at Relaxin Family Peptide Receptor 3. Ligand-directed signaling bias (as described in section II.C.2) has been described for relaxin-3, relaxin (van der Westhuizen et al., 2005), and the RXFP3 antagonist R3(B $\Delta 23-27) \mathrm{R} / \mathrm{I} 5$ (Kocan et al., 2014). The first indication of a functional relaxinRXFP3 interaction came from examination of metabolic responses recorded by microphysiometry (van der Westhuizen et al., 2005). In this study, human relaxin caused a small change in the extracellular acidification rate in CHO-RXFP3 cells. In CHO-K1, HEK293, and SN56 cell backgrounds, subsequent studies demonstrated that human relaxin, porcine relaxin, and human INSL3 caused weak inhibition of forskolin-stimulated cAMP accumulation compared with the strong inhibition mediated by human relaxin-3 (van der Westhuizen et al., 2010) (Table 3). Interestingly, the ability of INSL3 to activate RXFP3 appears to be specific for the human but not the mouse receptor (van der Westhuizen et al., 2010). Previous studies reported no inhibition of cAMP accumulation by either porcine relaxin or human INSL3 (Liu et al., 2003b), but because sensitivity of inhibitory cAMP assays is highly dependent on both the degree of activation of AC by forskolin and the time of stimulation, the differences observed most likely result from distinct experimental paradigms.

Similar to relaxin-3, relaxin and porcine relaxin also caused AP-1 reporter gene activation (van der Westhuizen et al., 2010) in CHO-K1, HEK293, and 

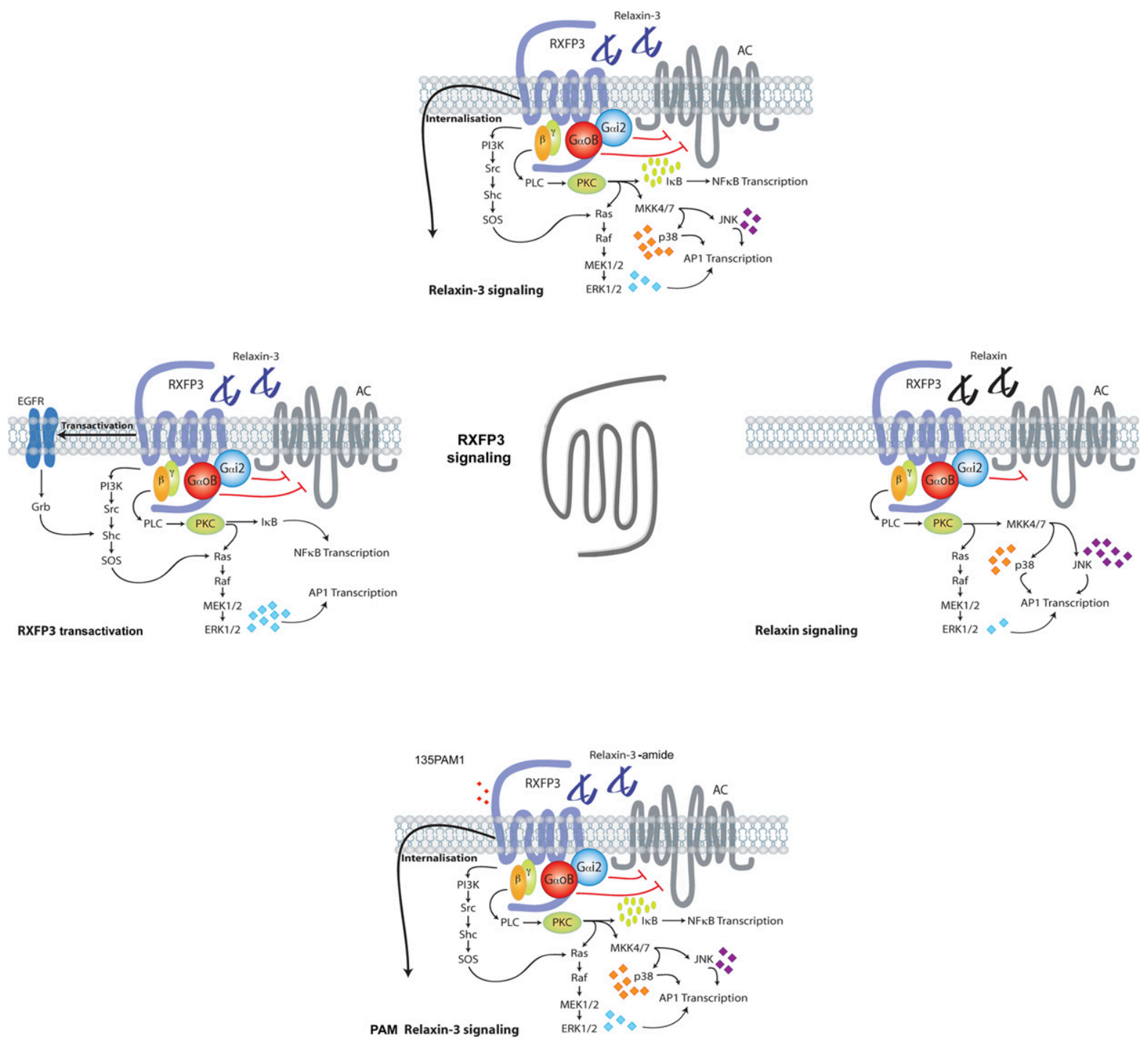

Fig. 5. Signal transduction pathways activated by RXFP3. The cognate ligand relaxin-3 activates RXFP3 that couples to G $\alpha_{\mathrm{i} / \mathrm{o}}$ proteins to inhibit adenylyl cyclase and activate ERK1/2 and p38MAPK phosphorylation to promote AP1 transcription. Stimulation of RXFP3 by relaxin activates only ERK1/2 and AP1 transcription. There is an allosteric site on RXFP3 that when occupied by a positive allosteric modulator 135PAM1 sensitizes responses to relaxin-3 amide. In tissues where they coexist, activation of RXFP3 may cause transactivation of the EGFR.

SN56 cell backgrounds, with an order of potency relaxin $>$ relaxin-3 $>$ porcine relaxin. Again, some AP-1 activation appears to be independent of $\mathrm{G} \alpha \alpha_{\mathrm{i} / \mathrm{o}}$ coupling, because pretreatment with PTX failed to block porcine relaxin-stimulated AP-1 reporter gene activation in CHO-RXFP3 cells. Thus, as for relaxin-3, porcine relaxin can activate $\mathrm{AP}-1$ reporter genes by a $\mathrm{G} \alpha_{\mathrm{i} / \mathrm{o}^{-}}$ independent mechanism, suggesting ligand-directed signaling bias (van der Westhuizen et al., 2010). In contrast, all of the AP-1 reporter gene responses observed after stimulation of RXFP3 in SN56 cells were blocked by PTX, suggesting that different pathways were involved in mediating the response downstream of the mouse RXFP3 receptor (van der Westhuizen et al., 2010).

In the CHO-K1 cell background, the relaxin-stimulated AP-1 response is strongly inhibited by the p38 MAPK inhibitor (RWJ67657) or the JNK inhibitor (SP600125), whereas the MEK inhibitor (PD98059) only weakly inhibited the AP-1 response, implicating p38 MAPK and JNK as the major MAPKs involved in the response to relaxin. In contrast in the HEK293 cell background, relaxin-stimulated AP-1 reporter gene activation was decreased after $\mathrm{p} 38$ MAPK or MEK inhibition but not by JNK inhibition, suggesting that p38 MAPK and ERK were the major MAPKs involved in mediating this response. In SN56 cells, endogenously expressing mouse RXFP3, p38 MAPK, JNK, or MEK inhibition was equally effective in blocking the response to relaxin, suggesting that all three kinases were equally important (van der Westhuizen et al., 2010). Clearly species and cell background are important determinants of the signaling pattern after RXFP3 activation. Direct measurement of pERK1/2, p38 MAPK, and pJNK after addition of relaxin family peptides has confirmed the findings of these inhibitor-based studies (Kocan et al., 2014) and demonstrates signaling bias; however, the $\mathrm{G} \alpha_{\mathrm{i} / 0}$-independent pathway remains to be 
identified for human relaxin-3 and porcine relaxin. Direct MAPK assays in CHO-RXFP3 cells show that human relaxin activates p38 MAPK and ERK1/2 with lower efficacy than human relaxin-3 but the two peptides have similar efficacy for JNK1/2/3 phosphorylation. Both human relaxin and human relaxin-3 activation of $\mathrm{p} 38$ MAPK, JNK1/2/3, or ERK1/2 involved PTX-sensitive $G$ proteins (van der Westhuizen et al., 2007, 2010; Kocan et al., 2014).

Evidence for signaling bias at RXFP3 also comes from studies utilizing an RXFP3 antagonist (Kocan et al., 2014). The RXFP3 antagonist R3(B $\Delta 23-27) R / I 5$ blocked human relaxin-3 AP-1 reporter gene activation but not human relaxin $\mathrm{AP}-1$ activation or human relaxin- $3 \mathrm{NF} \kappa \mathrm{B}$ activation. The antagonist itself R3(B $\Delta 23-27) \mathrm{R} / \mathrm{I} 5$ activated the SRE reporter but did not inhibit either human relaxin or human relaxin-3 SRE activation. Finally, although the antagonist R3(B $\Delta 23-27) \mathrm{R} / \mathrm{I} 5$ blocked human relaxin-3-stimulated p38MAPK and ERK1/2 phosphorylation, it was also a weak partial agonist for p38MAPK and ERK1/2 signaling (Table 3). Interestingly, in findings similar to previous studies, p38MAPK activation by $\mathrm{R} 3(\mathrm{~B} \Delta 23-27) \mathrm{R} / \mathrm{I} 5$ was found to be $\mathrm{G}$ protein independent. Direct examination of interactions between RXFP3 and G proteins using BRET showed that human relaxin-3-activated RXFP3 interacts with $\mathrm{G} \alpha_{\mathrm{i} 2}, \mathrm{G} \alpha_{\mathrm{i} 3}$, $\mathrm{G} \alpha_{\mathrm{oA}}$, and $\mathrm{G} \alpha_{\mathrm{oB}}$, whereas human relaxin or $\mathrm{R} 3(\mathrm{~B} \Delta 23-27)$ $\mathrm{R} / \mathrm{I} 5$ can only induce interactions with $\mathrm{G} \alpha_{\mathrm{i} 2}$ or $\mathrm{G} \alpha_{\mathrm{oB}}$. Interestingly, only human relaxin-3 promoted RXFP3/ $\beta$-arrestin interactions that were blocked by $\mathrm{R} 3(\mathrm{~B} \Delta 23-$ 27)R/I5 (Kocan et al., 2014). This is compelling evidence for ligand-directed signaling bias at RXFP3.

3. Allosteric Modulation of Relaxin Family Peptide Receptor 3. The RXFP3 allosteric modulator 135PAM1 has been examined only in receptor binding studies and in recombinant systems expressing the chimeric $\mathrm{G}$ protein $\mathrm{G} \alpha_{\mathrm{qI} 5}$ or a CRE reporter gene (Alvarez-Jaimes et al., 2012). It is not known what pattern of signaling is observed in systems that naturally express RXFP3, and given that 135PAM1 has poor solubility and displays selectivity for the C-terminal amides that are not naturally occurring, it has limited use experimentally (AlvarezJaimes et al., 2012).

\section{Relaxin Family Peptide Receptor 4 Signaling}

1. Canonical Signaling Pathways. Stimulation of cells expressing human RXFP4 by INSL5 or human relaxin-3 increases GTP $\gamma \mathrm{S}$ binding and inhibits forskolin-stimulated cAMP accumulation, suggesting that RXFP4 is $\mathrm{G} \alpha_{\mathrm{i} / \mathrm{o}}$ coupled (Liu et al., 2003a, 2005b). Coexpression of RXFP4 with the promiscuous $\mathrm{G}$ protein $\mathrm{G} \alpha_{16}$ in HEK293 cells produces a strong calcium signal in response to INSL5 and human relaxin-3 (Liu et al., 2003a, 2005b) that is not present in normal cells. Although relatively little information exists at present regarding RXFP4 signaling, it is expected that this will rapidly change given the identification of INSL5 as an incretin (Grosse et al., 2014).
Thus both RXFP3 and RXFP4 are $\mathrm{G} \alpha_{\mathrm{i} / \mathrm{o}}$-coupled receptors that show GTP $\gamma \mathrm{S}$ binding and inhibition of cAMP accumulation. More extensive studies of RXFP3 signaling have also revealed coupling to ERK1/2, p38MAPK, and JNK as well as ligand-directed signaling bias and allosteric modulation.

\section{Receptor-Protein Signaling and Regulatory Complexes}

It is increasingly recognized that GPCRs are important components of protein complexes and that this close relationship with other proteins affects how the receptor signals and is regulated. Receptor complexes can be constitutive (see section III.A.3) or may form after activation by agonists. Few studies have examined relaxin family peptide receptors in this light, and substantial information exists for only RXFP1 and to a lesser extent RXFP3.

\section{A. Relaxin Family Peptide Receptor 1}

1. G Proteins That Couple to Relaxin Family Peptide Receptor 1. Although it has long been assumed that stimulation of RXFP1 produces an increase in cAMP by $\mathrm{G} \alpha_{\mathrm{s}}$ activation of $\mathrm{AC}$, a direct functional interaction between RXFP1 and $\mathrm{G} \alpha_{\mathrm{s}}$ has been demonstrated only recently. Application of a specific $\mathrm{G} \alpha_{\mathrm{s}}$ inhibitor (NF449; $4,4^{\prime}, 4^{\prime \prime}, 4^{\prime \prime \prime}$-[carbonylbis(imino-5,1,3-benzenetriyl-bis (carbonylimino))]tetrakis-1,3-benzenedisulfonic acid, octasodium salt) to HEK293 cells expressing RXFP1 significantly decreased receptor-stimulated cAMP accumulation, whereas a low concentration of cholera toxin (a $\mathrm{G} \alpha_{\mathrm{S}}$ activator) substantially enhanced the cAMP response to relaxin (Halls and Cooper, 2010). Other supporting evidence includes the increase in cAMP after PTX pretreatment in the early phase $(<10$ minutes $)$ after stimulation of RXFP1. Furthermore, peptide fragments of ICL3 of RXFP1 (minimum length 615-629) added to rat tissues (striatum, cardiac, and skeletal muscle membranes) increased cAMP and "antagonized" the response to relaxin, whereas addition of peptide fragments of $\mathrm{G} \alpha_{\mathrm{S}}$ "antagonized" both relaxin stimulation and RXFP1ICL3 peptide stimulation of rat tissues (Shpakov et al., 2007). Many of the responses linked to RXFP1 display sensitivity to PTX (Halls et al., 2006), suggesting coupling to $\mathrm{G} \alpha_{\mathrm{i} / \mathrm{o}}$ proteins, and the specificity of the receptor for particular $\mathrm{G} \alpha_{\mathrm{i} / \mathrm{o}}$ isoforms was identified using PTXinsensitive G proteins (Halls et al., 2006). In HEK293 cells stably expressing RXFP1, restoration of the expected cAMP signaling profiles (after PTX pretreatment) occurred only with the expression of $\mathrm{G} \alpha_{\mathrm{oB}}$ and $\mathrm{G} \alpha_{\mathrm{i} 3}$. Activation of $\mathrm{G} \alpha_{\mathrm{oB}}$ and $\mathrm{G} \alpha_{\mathrm{i} 3}$ by RXFP1 was subsequently confirmed using $\left[{ }^{35} \mathrm{~S}\right] \mathrm{GTP} \gamma \mathrm{S}$ immunoprecipitation, and these studies also demonstrated that activation of $\mathrm{G} \alpha_{\mathrm{i} 3}$ occurs within 3 minutes of relaxin stimulation, suggesting that the observed delay in activation of the $\mathrm{G} \alpha_{\mathrm{i} 3}$-cAMP pathway occurs downstream of receptor coupling to this 
G protein (Halls et al., 2009b). Subsequent studies using a site-directed mutagenesis approach demonstrated that activation of the $\mathrm{G} \alpha_{\mathrm{i} 3}$-cAMP pathway involves the final 10 amino acids of the RXFP1 C terminus and in particular R752 (Halls et al., 2009b). Taken together, this suggests that all $\mathrm{G}$ protein coupling occurs rapidly after receptor activation and that $\mathrm{G} \alpha_{\mathrm{S}}$ (and probably $\mathrm{G} \alpha_{\mathrm{oB}}$ ) couple to RXFP1 within the ICL3, whereas coupling to $\mathrm{G} \alpha_{\mathrm{i} 3}$ is dependent upon the final 10 amino acids of the receptor C terminus.

2. Interactions between Relaxin Family Peptide Receptor 1 and $\beta$-Arrestins. $\beta$-Arrestins were initially identified for their role in receptor desensitization and internalization. Prototypical GPCR signaling is terminated after activation by receptor phosphorylation, $\beta$-arrestin binding followed by uncoupling from the $\mathrm{G}$ protein, and internalization (Luttrell and Gesty-Palmer, 2010). However $\beta$-arrestins are increasingly recognized as scaffold proteins that recruit a variety of catalytically active proteins that influence a wide range of signaling pathways, protein translation, and gene transcription. Unlike many other GPCRs, when expressed in HEK293T or Cos-7 cells, RXFP1 activation does not result in significant receptor phosphorylation, desensitization, or internalization (Callander et al., 2009). Little phosphorylation and internalization could be measured by whole cell radioligand binding, and there was minimal cell surface localization of GFP-tagged $\beta$-arrestin visualized by confocal imaging (Callander et al., 2009). In human primary decidual cells or HEK293 cells stably expressing RXFP1, there is weak internalization as measured by cell surface enzyme-linked immunosorbant assay (ELISA) that is enhanced by overexpression of $\beta$-arrestin-2 (Kern and Bryant-Greenwood, 2009). Constitutive interaction between RXFP1 and $\beta$-arrestin- 2 has been identified in the RXFP1 signalosome (Halls and Cooper, 2010), where $\beta$-arrestin-2 scaffolds PKA and PDE4D3 to regulate cAMP levels formed in response to subpicomolar concentrations of relaxin (see section III.A.3 for details). The signalosome dissociates in response to nanomolar concentrations of relaxin, and $\beta$-arrestin- 2 has no role in modulating canonical RXFP1 signaling. Thus, there does appear to be a significant role for $\beta$-arrestins in RXFP1 signaling after receptor activation, because there is little $\beta$-arrestin recruitment and minimal receptor internalization.

\section{B. Relaxin Family Peptide Receptor 2}

1. G Proteins That Couple to Relaxin Family Peptide Receptor 2. By using the same approach as that used for RXFP1, the specificity of RXFP2 for particular $\mathrm{G} \alpha_{\mathrm{i} / \mathrm{o}}$ isoforms was identified using PTX-insensitive G protein mutants (Halls et al., 2006). After expression of three $\mathrm{G} \alpha_{\mathrm{i}}$ and two $\mathrm{G} \alpha_{\mathrm{o}}$ isoforms in HEK293 cells stably expressing RXFP2, the restoration of the expected cAMP signaling profile (after PTX pretreatment) occurred only with $\mathrm{G} \alpha_{\mathrm{oB}}$ expression. This was subsequently confirmed using $\left[{ }^{35} \mathrm{~S}\right] \mathrm{GTP} \gamma \mathrm{S}$ immunoprecipitation (Halls et al., 2009b). Unlike RXFP1, RXFP2 does not couple to $\mathrm{G} \alpha_{\mathrm{i} 3}$; this is likely because receptor coupling to $\mathrm{G} \alpha_{\mathrm{i} 3}$ requires the final 10 amino acids of the RXFP1 C terminus (specifically R752) that are missing in RXFP2 (Halls et al., 2009b). It is highly likely that in a manner similar to RXFP1, RXFP2 also couples to $\mathrm{G} \alpha_{\mathrm{s}}$, because PTX pretreatment increases the cAMP generated after stimulation.

Both RXFP1 and RXFP2 couple to $\mathrm{G} \alpha_{\mathrm{s}}$ and $\mathrm{G} \alpha_{\mathrm{oB}}$, but only RXFP1 also couples to $\mathrm{G} \alpha_{\mathrm{i} 3}$. There is constitutive interaction between RXFP1 and $\beta$-arrestin- 2 in the signalosome that dissociates with the advent of canonical signaling. There is only minimal internalization after activation of RXFP1, suggesting little interaction with $\beta$-arrestin- 2 after signalosome dissociation. RXFP2 does not appear to interact with $\beta$-arrestins or internalize (Callander et al., 2009).

\section{Relaxin Family Peptide Receptor 3}

1. G Proteins That Couple to Relaxin Family Peptide Receptor 3 after Activation by Human Relaxin-3. The early deorphanization studies suggested that RXFP3 was a $\mathrm{G} \alpha_{\mathrm{i} / \mathrm{o}}$-coupled receptor that inhibited forskolin-stimulated cAMP accumulation (Liu et al., 2003b; van der Westhuizen et al., 2010). This was supported by studies in the cytosensor microphysiometer, where pretreatment of CHORXFP3 cells with PTX strongly inhibited the extracellular acidification rate, suggesting that the signaling pathways activated by RXFP3 were downstream of $\mathrm{G} \alpha \alpha_{\mathrm{i} / \mathrm{o}}$ (van der Westhuizen et al., 2005). As for RXFP1 and RXFP2, PTX-insensitive G proteins were used to determine the particular $G$ proteins involved (van der Westhuizen, 2008). PTX pretreatment completed abrogated the ERK1/2 response in both CHO-RXFP3 and HEK-RXFP3 cells. In CHO-RXFP3 cells, the response was partially restored by transfection of $\mathrm{G} \alpha_{\mathrm{i} 2}$ or $\mathrm{G} \alpha_{\mathrm{oB}}$ only. Similarly, in HEK-RXFP3 cells, signaling was partially restored by expression of mutant $\mathrm{G} \alpha_{\mathrm{i} 2}$ or $\mathrm{G} \alpha_{\mathrm{oB}}$ but also by mutant $\mathrm{G} \alpha_{\mathrm{oA}}$. These differences may relate to different colocalization of receptors and $G$ proteins in particular cell types. More recent studies used BRET to investigate ligand-induced interactions between RXFP3-RLuc8 and G proteins (Gr2-Venus) in live cells. Similar to previous studies, treatment of CHO-RXFP3-RLuc8 cells with human relaxin-3 caused activation of $\mathrm{G} \alpha_{\mathrm{i} 2}, \mathrm{G} \alpha_{\mathrm{oA}}$, and $\mathrm{G} \alpha_{\mathrm{oB}}$, but also revealed an interaction between RXFP3 and $\mathrm{G} \alpha_{\mathrm{i} 3}$ (Kocan et al., 2014). Thus RXFP3 has the potential to couple to $\mathrm{G} \alpha_{\mathrm{i} 2}$, $\mathrm{G} \alpha_{\mathrm{i} 3}, \mathrm{G} \alpha_{\mathrm{oA}}$, and $\mathrm{G} \alpha_{\mathrm{oB}}$ after activation by human relaxin-3, but the receptor preferentially couples to discrete subsets of these $\mathrm{G}$ proteins dependent on cell type.

2. G Proteins That Couple to Relaxin Family Peptide Receptor 3 after Activation by the Biased Ligands Human Relaxin and $R 3(B \Delta 23-27) R / I 5$. The different signaling patterns observed with the biased ligands human relaxin and R3(B $\Delta 23-27) R / 15$ (see section III.C.2 for details) were also reflected in a distinct pattern of $\mathrm{G}$ protein coupling in 
BRET studies (Kocan et al., 2014). The biased ligands coupled only with $\mathrm{G} \alpha_{\mathrm{i} 2}$ or $\mathrm{G} \alpha_{\mathrm{oB}}$, and the signal was much lower than that observed for human relaxin-3 (Kocan et al., 2014). To determine the potential biologic relevance of these findings, studies will be needed in cells that express RXFP3 at physiologic levels.

3. Interactions between Relaxin Family Peptide Receptor 3 and $\beta$-Arrestins. Interactions between RXFP3 and $\beta$-arrestins occur after administration of human relaxin-3 but not relaxin or the RXFP3 antagonist R3 (B $\Delta 23-27) \mathrm{R} / \mathrm{I} 5$ as measured by real-time kinetic BRET between RXFP3-Rluc8 and a $\beta$-arrestin fusion protein ( $\beta$-arrestin-1-Venus or $\beta$-arrestin-2-Venus). Preincubation with R3(B $\Delta 2327) \mathrm{R} / \mathrm{I} 5$ completely inhibits the human relaxin-3 stimulated recruitment of $\beta$-arrestin- 1 and $\beta$-arrestin-2 to RXFP3 (Kocan et al., 2014). Furthermore, PTX pretreatment partially blocked $\beta$-arrestin recruitment, suggesting that RXFP3 recruits $\beta$-arrestins via $\mathrm{G} \alpha_{\mathrm{i} / \mathrm{o}}$-dependent and -independent pathways. The involvement of $\beta$-arrestin in ERK1/2 activation downstream of RXFP3 was investigated by comparing cells expressing dominant negative (V53D) versus wild-type (WT) $\beta$-arrestin-1. There was no inhibitory effect of the dominant negative $\beta$-arrestin- 1 on ERK1/2 activation, suggesting that, unlike several other GPCRs (Cottrell et al., 2009; Luttrell and Gesty-Palmer, 2010), the RXFP3/ $\beta$-arrestin- 1 interaction contributes little to ERK1/2 signaling (Kocan et al., 2014).

Previous studies demonstrated internalization of RXFP3 after 10 minutes of stimulation with human relaxin-3 but not human relaxin, porcine relaxin, or INSL3 as assessed by radioligand internalization assays ( $70-90 \%$ of receptors internalized) and confocal microscopy (van der Westhuizen et al., 2010). Taken together, these studies suggest that treatment with only the cognate ligand human relaxin-3 causes RXFP3 to undergo classic $\beta$-arrestin-dependent internalization. The detailed mechanisms involved in RXFP3 internalization, phosphorylation, and recycling/ degradation all remain to be determined.

\section{Relaxin Family Peptide Receptor 4}

\section{G Proteins That Couple to Relaxin Family Peptide} Receptor 4. There is currently a paucity of information regarding signaling pathways activated by RXFP4. The receptor is $\mathrm{G}$ protein-coupled because activation by INSL5 or human relaxin-3 increases GTP $\gamma \mathrm{S}$ binding. cAMP accumulation in response to forskolin is inhibited, suggesting that RXFP4 couples to $\mathrm{G} \alpha_{\mathrm{i} / \mathrm{o}}$ proteins (Liu et al., 2003a, 2005b). Calcium signaling is activated only in cells coexpressing RXFP4 and the promiscuous $\mathrm{G}$ protein $\mathrm{G} \alpha_{16}$. Direct evidence for RXFP4-G protein coupling is currently lacking.

In contrast to RXFP1, the information available on proteins interacting with RXFP3 and RXFP4 is largely confined to $\mathrm{G}$ proteins and $\beta$-arrestins. RXFP3 couples to $\mathrm{G} \alpha_{\mathrm{oA}}, \mathrm{G} \alpha_{\mathrm{oB}} \mathrm{G} \alpha_{\mathrm{i} 2}$, and $\mathrm{G} \alpha_{\mathrm{i} 3}$, although the pattern observed varies with cell type and ligand. There is also good evidence for an interaction of RXFP3 with $\beta$-arrestins that modulates receptor internalization. At present, RXFP4 is known to couple only to proteins of the $\mathrm{G} \alpha_{\mathrm{j} / \mathrm{o}}$ family.

\section{Physiologic Roles of Relaxin Family Peptide Receptors and Their Cognate Ligands}

The physiologic roles of relaxin family peptides and their receptors have been extensively reviewed (Ivell and Anand-Ivell, 2009; Smith et al., 2011; Bathgate et al., 2013a). In particular, the role of relaxin in female and male reproductive physiology have been well studied (Sherwood, 1994, 2004; Bathgate et al., 2013a, 2006c). For this reason, only brief overviews will be given here.

\section{A. Relaxin and Relaxin Family Peptide Receptor 1}

1. Reproduction. The discovery of relaxin in 1926 (Hisaw, 1926) and the demonstration of its effects on the pubic ligament led to the peptide being regarded as a hormone of reproduction for many years. Subsequent studies showed that relaxin produced by the corpus luteum and/or placenta has important roles in pregnancy and parturition and is a major circulating hormone during pregnancy in all mammalian species acting on the pubic symphysis, cervix, uterus, vagina, and mammary glands. It also is responsible for many of the cardiovascular changes that occur during pregnancy (Debrah et al., 2006; Conrad, 2011).

Relaxin causes increased flexibility and elasticity of the interpubic ligament during pregnancy in several species (O'Connor et al., 1966; Steinetz et al., 1983), and in the relaxin knockout mouse the interpubic ligament fails to develop (Zhao et al., 1999). The peptide also causes softening and hypertrophy of the cervix during the second half of pregnancy in mammals (Bathgate et al., 2006c), and these effects are impaired in both relaxin-deficient rats (Burger and Sherwood, 1998) and relaxin knockout mice (Zhao et al., 1999; Bathgate et al., 2006c). In humans, although relaxin levels increase during cervical ripening, this still occurs after embryo transfer where circulating relaxin levels are undetectable (Eddie et al., 1990a). In clinical trials, although direct application of porcine relaxin to the cervix appeared to assist in ripening (Evans et al., 1983; MacLennan et al., 1986b), later studies with recombinant human relaxin failed to confirm this finding (Bell et al., 1993; Weiss et al., 2009).

Relaxin reduces uterine contractility (Krantz et al., 1950) and increases uterine growth (Steinetz et al., 1957) in rat, mouse, guinea pig, hamster, and pig (Bathgate et al., 2006c) but in humans, has little effect on uterine tone (MacLennan et al., 1986a, 1995). In humans, relaxin is probably important for implantation rather than the later stages of pregnancy. Relaxin promotes growth of the vagina during pregnancy in mice (Hall, 1960; Schink and Struck, 1968) and rats (Burger and Sherwood, 1995; Zhao et al., 1996, 2001), and consistent with these effects, the growth of the vagina was profoundly decreased in 
relaxin knockout mice (Zhao et al., 2000). These effects are essential for normal delivery in some species but not humans.

Relaxin has trophic effects on the mammary gland and/or nipple in several species. Nipple development is completely blocked in relaxin knockout mice with limited effects on the mammary gland (Zhao et al., 1999). Pups born to relaxin-knockout mice die within 24 hours unless crossfostered to wild-type mothers, an effect entirely due to poor nipple development, because female knockout mice produce milk normally (Zhao et al., 1999). The same phenotype is displayed by RXFP1 knockout mice (KrajncFranken et al., 2004) and is not rescued by transgenic overexpression of INSL3 (Kamat et al., 2004). Relaxin binding sites are present in the mammary glands of pigs, rats, and humans; in humans, RXFP1 receptors are localized to the nipple, epithelial cells (Kohsaka et al., 1998), and stromal tissue (Ivell et al., 2003).

Although relaxin has many well defined roles in reproduction in many species, in humans these effects are often absent or ill defined. Relaxin is produced in the human ovary during the luteal phase of the menstrual cycle and secreted into the circulation (Eddie et al., 1990b; Stewart et al., 1990; Chen et al., 2003). In the clinical trial of relaxin for the treatment of scleroderma (Erikson and Unemori, 2001), women received 24-week subcutaneous infusion of relaxin and reported heavy, irregular, or prolonged menstrual bleeding (Unemori et al., 1999). In humans and other primates, the peak of relaxin secretion is in the first trimester of pregnancy. However relaxin is clearly not mandatory during the induction or maintenance of pregnancy, because women without ovaries can become pregnant by ovum donation even though they have undetectable levels of circulating relaxin (Johnson et al., 1991). Relaxin secretion also correlates with increased expression of RXFP1 mRNA and relaxin binding in the human endometrium in the secretory phase of the menstrual cycle (Bond et al., 2004; Campitiello et al., 2011).

Perhaps somewhat surprisingly given its history as a hormone acting on the female reproductive tract, relaxin is also found in the male reproductive tract in most mammals. Relaxin from human seminal plasma is identical to luteal relaxin (Winslow et al., 1992) and appears to be produced in the prostate (Yki-Järvinen et al., 1983; Sokol et al., 1989). RXFP1 is expressed in sperm in both mice (Krajnc-Franken et al., 2004) and humans (Carrell et al., 1995; Ferlin et al., 2012) and increases sperm motility and facilitates penetration into oocytes (Weiss, 1989). Another potential role for seminal relaxin may be to act on the female reproductive tract to prepare the endometrium for implantation (Telgmann and Gellersen, 1998; Unemori et al., 1999; Anand-Ivell and Ivell, 2014).

2. Central Nervous System. In mammals, there are high concentrations of RXFP1 in the SFO and OVLT that are activated by relaxin to cause a reduction in plasma osmolality (Sunn et al., 2002). This effect in rats is associated with increased serum relaxin levels during the second half of pregnancy (Sherwood et al., 1980; Lindheimer et al., 1989) and is absent in pregnant rats that have undergone ovariectomy or treatment with relaxin antibodies (Novak et al., 2001). Similarly, the decrease in plasma osmolality with late pregnancy is not observed in relaxin knockout mice (Zhao et al., 1999). In humans, there is a decrease in plasma osmolality with pregnancy, but this may not be completely attributable to relaxin because women who become pregnant after ovum donation (and hence lack circulating relaxin) display attenuated (Smith et al., 2006b) or no decrease in plasma osmolality (Johnson et al., 1991, 1996). However, the apparent lack of relaxin in the absence of a corpus luteum may have to be reassessed using more sensitive methods of detection since the discovery of responses to femtomolar concentrations of relaxin (Halls and Cooper, 2010), well below the level of detection using current ELISA methods; relaxin is also produced locally in many tissues including the uterus. Relaxin administered intracerebroventricularly or intravenously is dipsogenic in nonpregnant rats (Summerlee et al., 1998a), and rats given relaxin monoclonal antibodies in the second half of pregnancy show a reduction in water consumption. Administration of porcine or human relaxin intravenously causes activation (increased $c$-fos expression) of neurons in the peripheral and dorsal segments of the SFO and in the dorsal cap region of the OVLT as well as in the supraoptic and paraventricular nuclei of the hypothalamus (McKinley et al., 1997, 1998; Sunn et al., 2001, 2002), all sites of localization of RXFP1 and accessible to circulating relaxin (Weisinger et al., 1993).

Relaxin acting on RXFP1 in the circumventricular organs and hypothalamic nuclei may have a role in the timing of parturition, because this is disrupted by central administration of a relaxin monoclonal antibody (Summerlee et al., 1998b). RXFP1 is also highly expressed in the basolateral amygdala, and administration of relaxin to this region impairs fear-related memory consolidation in rats (Ma et al., 2005). However, no specific agonist or antagonist studies have been carried out to determine whether these effects are mediated by RXFP1 or to determine the source of endogenous relaxin that activates these receptors (Callander and Bathgate, 2010). Although RXFP1 is also highly expressed in other regions associated with memory formation, such as the neocortex, thalamic nuclei, hippocampus, and supramammillary nucleus, there are no studies to date that examine effects on memory. RXFP1 is also present in the oxytocin-containing cells of the paraventricular and supraoptic hypothalamic nuclei (Burazin et al., 2005), and intravenous relaxin administration increases oxytocin neuron activity and oxytocin release (Way and Leng, 1992)

3. Blood Vessels. Relaxin plays an important role in many of the adaptive cardiovascular changes that occur in pregnancy (Conrad, 2011). These include increases in cardiac output, heart rate, and a decrease in vascular 
resistance (Conrad et al., 2004; Conrad and Novak, 2004; Debrah et al., 2005, 2006). Increases in plasma volume and decreased blood pressure are not consistently observed (Ahokas et al., 1989; Conrad, 2004, 2010, 2011). Ovariectomy or passive immunization with monoclonal antibodies for rat relaxin prevent these adaptive changes (Novak et al., 2001). In both female and male rats, chronic relaxin administration (osmotic minipump) increases renal plasma flow and glomerular filtration rate (Danielson et al., 1999). In humans, a few studies suggest similar effects of relaxin on the cardiovascular system (Conrad and Shroff, 2011). In the clinical trial for scleroderma, long-term (6 months) infusion of relaxin increased creatinine clearance and modestly decreased blood pressure (Erikson and Unemori, 2001; Teichman et al., 2009). In the pilot trial in patients suffering from stable chronic heart failure, a short (24 hours) infusion of relaxin was associated with decreased systemic and pulmonary vascular resistance and pulmonary wedge pressure and elevated cardiac index without significantly affecting systolic or mean blood pressure (Dschietzig et al., 2009c; Teichman et al., 2009).

Vasodilation in arterioles, capillaries, and venules is a common response to relaxin in reproductive tissues (Vasilenko et al., 1986; Bani et al., 1988; Lee et al., 1992), heart (Bani-Sacchi et al., 1995; Masini et al., 1997; Bani et al., 1998b), liver (Bani et al., 2001), and cecum (Bigazzi et al., 1986). Relaxin is a potent vasodilator in arteries (Conrad, 2010; McGuane et al., 2011b), although the effect is vessel specific (McGuane et al., 2011b). Relaxin is a physiologic antagonist of vasoconstrictors in mesenteric arteries (St-Louis and Massicotte, 1985; Massicotte et al., 1989), primary bovine aortic smooth muscle cells (Bani et al., 1998a), and uterine artery (Longo et al., 2003). The vasodilator effects in guinea pig and rat coronary arteries and in bovine cultured smooth muscle cells are associated with increased NO synthesis (Bani-Sacchi et al., 1995; Bani et al., 1998a). The rise in intracellular calcium produced by $\alpha$-thrombin or angiotensin II is also reduced by relaxin (Bani et al., 1998a; Failli et al., 2002). In humans, there are vasodilator effects in gluteal resistance or subcutaneous arteries but little or no effect in pulmonary, myometrial, or placental vessels (Petersen et al., 1991; Fisher et al., 2002; McGuane et al., 2011b). In gluteal arteries, the vasodilator responses likely involve NO and interestingly were influenced by the medication being administered to patients. Arteries obtained from patients on angiotensin converting enzyme inhibitors showed marked attenuation of the vasodilator response to relaxin, effects that appeared to be further enhanced by inhibition of cyclooxygenase (Fisher et al., 2002). However, in patients not receiving angiotensin converting enzyme inhibitors, inhibition of cyclooxygenase had little effect on the vasodilator response to relaxin (Fisher et al., 2002). The vasodilator mechanisms suggested for relaxin in both humans and in animal models involve activation of NOS (Nistri and Bani, 2003; Conrad and Novak, 2004), VEGF, placental growth factor (PGF), matrix metalloproteinases, $\mathrm{ET}_{\mathrm{B}}$ receptors (Novak et al., 2002; Dschietzig et al., 2003), and modification of the extracellular matrix of the vessel walls (Jeyabalan et al., 2003; Lekgabe et al., 2005; Xu et al., 2010; McGuane et al., 2011b). These responses have a distinct temporal hierarchy. The acute (within minutes) responses are endothelium dependent and blocked by NOS inhibitors, the PI3K inhibitors Wortmannin and LY294002, and by PTX pretreatment but not by the VEGF receptor antagonist SU5416 [(3Z)-3-[(3,5-dimethyl$1 H$-pyrrol-2-yl)methylidene]-1,3-dihydro- $2 H$-indol-2-one] (McGuane et al., 2011b). The effects are consistent with G $\beta \gamma$ activation of PI3K, Akt phosphorylation, and eNOS. Treatment with NOS inhibitors or removal of the endothelium enhances contractions of vascular smooth muscle to agonists in both pregnant rats (Danielson and Conrad, 1995; Gandley et al., 2001) and in rats treated chronically with relaxin (Novak et al., 2002). This does not appear to be attributable to changes in NOS expression, because alterations in renal hemodynamics in pregnancy are not accompanied by large changes in NOS expression. In pregnant rats, eNOS expression in the renal artery fell by $39 \%$, whereas iNOS and nNOS expression increased by 31 and 25\%, respectively (Alexander et al., 1999).

Longer-term (within hours) vasodilator responses to relaxin also involve NOS, because blockade with NG-monomethyl-L-arginine prevents the renal hemodynamic and hyperfiltration responses (Danielson et al., 1999), and these effects can be recapitulated in vitro (Novak et al., 2002). The longer-term response involves endothelial $\mathrm{ET}_{\mathrm{B}}$ receptors that release NO (Dschietzig et al., 2003; Jeyabalan et al., 2003). Two mechanisms involving the relaxin- $\mathrm{ET}_{\mathrm{B}}$ receptor pathway have been found. In rodent and human vessels of $\sim 100-300 \mu \mathrm{m}$ diameter, relaxin activates MMP-9 and later also MMP-2 to generate ET-1(1-32), rather than ET-1(1-21), from big ET-1 (Jeyabalan et al., 2003). The effect of MMPs on big ET-1 has been described independently of relaxin (Fernandez-Patron et al., 1999). ET-1(1-32) then preferentially binds to the endothelial $\mathrm{ET}_{\mathrm{B}}$ receptor causing NO release. In blood vessels from pregnant or relaxintreated nonpregnant rats, pro-MMP-2 and MMP-2 activity and pro-MMP-2 protein and mRNA are increased (Jeyabalan et al., 2003, 2006). MMP-9 activity also appears to be increased somewhat, although more recent studies suggest that MMP-9 is more important in the relatively short-term responses to relaxin (4-6 hours) with reversal of the effects being produced by MMP-9 rather than MMP-2-neutralizing antibodies (Jeyabalan et al., 2007). This is supported by studies with the selective MMP-2 inhibitor cyclic CTTHWGFTLC, the MMP inhibitor GM6001 [(2R)- $N^{\prime}$-hydroxy- $N$-[(2S)-3-(5Hindol-3-yl)-1-methylamino-1-oxopropan-2-yl]-2-(2-methylpropyl)butanediamide], or a TIMP-2 or MMP-2 neutralizing antibody, all of which inhibit the vasodilator actions of relaxin in renal arteries (Jeyabalan et al., 2003), 
whereas inhibition of the formation of ET(1-21) by phosphoramidon has no effect. These studies clearly implicate MMPs in the vasodilator actions of relaxin, although MMPs also cleave calcitonin gene-related peptide to promote vasoconstriction (Fernandez-Patron et al., 2000). These findings correspond well with those obtained with the $\mathrm{ET}_{\mathrm{B}}$ antagonist RES-701-1 [cyclic (Gly1-Asp9) (Gly-Asn-Trp-His-Gly-Thr-Ala-Pro-Asp-Trp-Phe-Phe-AsnTyr-Tyr-Trp)] that blocks renal hemodynamic changes produced by relaxin (Danielson et al., 2000) in rats and antagonizes inhibition of renal artery smooth muscle produced by relaxin or in pregnancy (Novak et al., 2002). Similar effects were produced in vitro by the ET receptor antagonist SB209670 [(+)-(1S,2R,3S)-3-(2-carboxymethoxy4methoxyphenyl)-1-(3,4-methylenedioxyphenyl)-5-(prop-1yloxy)indane-2-carboxylic acid] but not by the $\mathrm{ET}_{\mathrm{A}}$ selective BQ123 (2-[(3R,6R,9S,12R,15S)-6-(1H-indol3-ylmethyl)-9-(2-methylpropyl)-2,5,8,11,14-pentaoxo12-propan-2-yl-1,4,7,10,13-pentazabicyclo[13.3.0]octadecan3-yl]acetic acid) (Gandley et al., 2001).

An alternative mechanism that occurs mainly in larger vessels and in the pulmonary circulation (Dschietzig et al., 2001a,b, 2003) involves relaxin-induced upregulation of endothelial $\mathrm{ET}_{\mathrm{B}}$ receptor requiring Raf-1, MEK-1, ERK-1/2, and $\mathrm{NF}_{\kappa} \mathrm{B}$ (Dschietzig et al., 2003). This mechanism spares the smooth muscle $\mathrm{ET}_{\mathrm{B}}$ receptor, a contributor to ET-1-related vasoconstriction. Thus, relaxin acts as a functional ET-1 antagonist, because the endothelial $\mathrm{ET}_{\mathrm{B}}$ receptor mediates $\mathrm{NO}$ production and, equally importantly, ET-1 clearance (Dschietzig et al., 2001b, 2003). Although another study does not support this mechanism (Kerchner et al., 2005), this can be explained by the use of small rather than large arteries, no pulmonary endothelial cells, and significantly lower relaxin concentrations.

Evidence also exists for a role of VEGF in the longerterm vasodilator actions of relaxin. In human endometrial cells, relaxin increases cAMP levels and VEGF expression, and the effects are prevented by $\mathrm{AC}$ inhibition and mimicked by forskolin or a PDE inhibitor (Unemori et al., 1999), suggesting that relaxin-stimulated cAMP production mediates increased VEGF transcription and, thus, angiogenesis. However, although treatment of rat or mouse renal arteries or human subcutaneous arteries with the VEGF receptor antagonist SU5416 blocks the vasorelaxant effects of relaxin (McGuane et al., 2011a), the specificity of SU5416 has been questioned (Arora and Scholar, 2005; Loges et al., 2006), and in rat renal arteries, SU5416 potentiates the acute vasodilator effects of relaxin (McGuane et al., 2011b). Furthermore, although VEGF-neutralizing antibodies block the effects of relaxin, this can also be mimicked by placental growth factor antibodies (McGuane et al., 2011a), again making interpretation of the results difficult. It is possible that the antibodies, by blocking the vasodilator effects of VEGF and PGF, may produce physiologic antagonism of the response to relaxin without necessarily being directly related to the primary mechanism of action. Alternatively, these results may reflect MMP-2- or MMP-9-mediated release of extracellular matrix-bound VEGF (and PGF) (Bergers et al., 2000; van Hinsbergh et al., 2006) that could underpin the MMP-ET $\mathrm{B}_{\mathrm{B}}$ pathway by stimulating expression of prepro-ET-1.

Finally, relaxin promotes angiogenesis (Unemori et al., 1999; Unemori et al., 2000) by inducing VEGF and basic fibroblast growth factor synthesis. These effects are likely important in promotion of wound healing (Bitto et al., 2013) and also in the heavy, irregular, or prolonged menstrual bleeding that accompanies relaxin administration (Unemori et al., 1999). Relaxin also increases arterial compliance by its effects on geometric remodeling (increases in unstressed wall area and wall-to-lumen area ratio) and compositional remodeling (decrease in collagen-to-total protein ratio) in certain arteries (Chan and Cipolla, 2011; Debrah et al., 2011; Gooi et al., 2013). These properties expedite tissue and organ perfusion in the long term.

Compared with arteries, remarkably little is known of the effects of relaxin on veins. It was reported that the vasodilator potency of relaxin is lower in veins than arteries ( $\mathrm{Li}$ et al., 2005). Rat mesenteric veins, in contrast to arteries, are not remodeled by relaxin (Jelinic et al., 2014). It is important to recognize that venous tone is significantly more dependent on sympathoadrenergic innervation and pacemaker cells (Cajal cells) than arterial tone (Gelman, 2008). Given the interest in the utility of vasodilators in the treatment of cardiac failure (Bhushan et al., 2014), this promises to be an important future area of research.

Relaxin protects against endothelial dysfunction that denotes an inflammatory and oxidative stress-related vascular pathology characterized by compromised vasodilator capacity on the basis of reduced NO bioavailability. In a rat in vitro model (Dschietzig et al., 2012) in which TNF- $\alpha$ impairs endothelial function, relaxin, by a mechanism involving PI3K and GR signaling, attenuates the upregulation of arginase-II that deprives NOS of its substrate L-arginine; antagonizes the increased ET-1 expression that promotes vasoconstriction and inflammation; upregulates SOD-1 expression to combat oxidative/nitrosative stress; and finally, causes partial reversal of dephosphorylation of eNOS, which impairs NO generation. Others have confirmed the SOD-1 finding and also reported elevated SOD-2 expression (Collino et al., 2013). This vascular protection is further complemented by NO-mediated inhibition of platelet and neutrophil activation (Bani et al., 1995a,b; Masini et al., 2004). Relaxin also attenuates VCAM-1 and MCP-1 stimulation that mediate the initial monocyte-endothelium contact and are both key events in early vascular inflammation (Brecht et al., 2011).

4. Heart. Relaxin acts directly on the heart. The presence of RXFP1 in the heart was first suggested by the demonstration of high-affinity binding sites for 
relaxin in rat atria (Osheroff et al., 1992; Osheroff and Ho, 1993; Tan et al., 1999). Subsequently, relaxin was shown to be a powerful inotropic and chronotropic agent (Kakouris et al., 1992). Positive chronotropic effects of relaxin occur in perfused intact hearts (Thomas and Vandlen, 1993; Bani-Sacchi et al., 1995; Coulson et al., 1996; Toth et al., 1996) and isolated right atria (Kakouris et al., 1992; Tan et al., 1998; Ward et al., 1992; Wade et al., 1994; Mathieu et al., 2001), and the positive inotropic effects occur in left atria (Kakouris et al., 1992; Ward et al., 1992; Wade et al., 1994; Tan et al., 1998; Mathieu et al., 2001). Chronotropic effects of relaxin are accompanied by the secretion of atrial natriuretic peptide in isolated perfused rat hearts (Toth et al., 1996). In rabbit sinoatrial node cells, relaxin increases the rate of spontaneous action potentials and the L-type calcium current (Han et al., 1994) by a PKA-dependent mechanism. In rat atrial myocytes, relaxin inhibits outward potassium currents, increases action potential duration, and enhances calcium entry; this is the mechanism underlying the positive inotropic effect (Piedras-Renteria et al., 1997a,b). Regarding inotropic effects in the ventricle, there is one report in mice (Shaw et al., 2009) indicating that relaxin increases myofilament calcium sensitivity by promoting phosphorylation of myosin-binding protein $\mathrm{C}$, troponin I, and troponin C. This results in higher force generation at normal calcium levels and is dependent on myofilament-associated $\mathrm{PKC} \delta$. This is the only report of inotropic responses to relaxin in ventricles. Although the cardiac actions of relaxin are largely confined to rodents, a recent study (Dschietzig et al., 2011) demonstrates inotropic effects in human atria (but not in ventricular myocardium), which are preserved in failing hearts and involve PKA, outward potassium currents, and PI3K. The effects of relaxin on the cardiovascular system are not gender specific.

Relaxin also protects against myocardial injury caused by ischemia and reperfusion (Bani et al., 1998b). Pretreatment of rats with relaxin 30 minutes before cardiac ischemia produced by ligating the left anterior descending coronary artery markedly reduced the size of the penumbra and reduced cardiac arrhythmias, mortality, myeloperoxidase activity, malonyldialdehyde production, and calcium content as well as causing an improved morphology (Bani et al., 1998b). These findings were confirmed in an in vivo pig model of myocardial infarction in which relaxin, administered during reperfusion of the left anterior descending coronary artery, attenuated leukocyte recruitment and oxidative damage and improved contractile recovery (Perna et al., 2005). In a mouse infarction model, relaxin improves postinfarction remodeling by suppressing reactive fibrosis in vital myocardium, without affecting reparative fibrosis (scarring) within the infarcted area (Samuel et al., 2011). This postinfarction fibrosis-modulating effect could also assist stem cell-based reparative therapies that appear to be promoted by the peptide. Mouse $\mathrm{C} 2 \mathrm{C} 12$ skeletal myoblasts engineered to overexpress human relaxin demonstrate growth and functional maturation of neonatal immature cardiomyocytes assessed by the expression of myocardiumspecific structural genes (connexin 43 , troponin $\mathrm{T}$, and HCN4 ion channel) (Formigli et al., 2009). Coronary infusion of $\mathrm{C} 2 \mathrm{C} 12$ skeletal myoblasts overexpressing relaxin in conjunction with administration of exogenous relaxin starting at day 30 after infarction in rats improves myocardial viability in the infarcted area compared with myoblast therapy alone (Bonacchi et al., 2009).

Relaxin also attenuates hypertrophy in rat neonatal cardiomyocytes by inhibiting myofibroblast activation and the subsequent paracrine release of growth factors (Moore et al., 2007), confirming an earlier study in spontaneously hypertensive rats and suggesting that endogenous relaxin has antihypertrophic actions (Dschietzig et al., 2005).

Atrial fibrillation, a common arrhythmia that develops during ageing-related fibrosis, hypertension, or heart failure, is suppressed by relaxin in spontaneously hypertensive rats, an established model of end-organ damage in response to hypertension (Parikh et al., 2013). The aforementioned effects of relaxin on atrial hypertrophy and fibrosis resulted in restoration of normal conduction velocity, a reduced action potential duration, and hence decreased atrial fibrillation.

Relaxin may be a naturally occurring cardioprotective agent, because in chronic heart failure, the expression of human relaxin-1 and human relaxin is increased in both atria and ventricles, and the level of expression correlates with the degree of failure (Dschietzig et al., 2001b; Fisher et al., 2003) but is not a predictor of clinical outcomes (Fisher et al., 2003). Increased circulating relaxin levels in human heart failure were not confirmed in two subsequent clinical studies (Kupari et al., 2005; Heringlake et al., 2009), possibly due to a methodological issue related to the utilization of a new batch of antiserum (Kupari et al., 2005; Heringlake et al., 2009) for the ELISA (Dschietzig et al., 2001b; Fisher et al., 2002). Increased relaxin gene expression in chronic heart failure was confirmed by two independent studies using the rat infarction model (Kompa et al., 2002; Zhang et al., 2005).

After the demonstration of protective actions in the cardiovascular system, relaxin was tested in human heart failure. A hemodynamic pilot study in patients with stable chronic heart failure (Dschietzig et al., 2009c) demonstrated that a 24-hour intravenous infusion of recombinant human relaxin markedly elevated cardiac index without affecting heart rate and decreased pulmonary wedge pressure, systemic, and pulmonary vascular resistance without affecting systolic or mean arterial blood pressure and central venous pressure. Hemodynamic changes occurred within 45 to 60 minutes, and relaxin infusion improved creatinine and blood urea nitrogen clearance and was free of relevant side effects. 
The use of relaxin for acute heart failure (defined as new onset or, more frequently, worsening of known heart failure) is promising. In the Pre-RELAX (phase II) and RELAX (phase III) trials (Teerlink et al., 2009, 2013), relaxin moderately improved dyspnea (the primary endpoint), was exceptionally safe, improved renal function, and lowered all-cause as well as cardiovascular mortality at day 180 . Because both trials were not powered statistically to assess mortality, another adequately powered mortality trial in acute heart failure is in progress. If successful, relaxin would become the first evidence-based remedy for this highly challenging clinical syndrome.

5. Kidney. The pronounced effects of relaxin on renal arteries imply a pivotal role in the regulation of renal function. Indeed, relaxin is the major renal vasodilator responsible for the increases in renal plasma flow and glomerular filtration rate during rat pregnancy (Novak et al., 2001). The increase in renal plasma flow and glomerular filtration rate with relaxin also occurs in nonpregnant female rats and male rats (Danielson et al., 1999; Danielson and Conrad, 2003) and is accompanied by natriuresis (Bogzil et al., 2005).

In humans, circumstantial evidence for a role for relaxin comes from a clinical study in which women who were pregnant through egg donation, and therefore relaxin deficient, exhibited a decreased glomerular filtration rate compared with that observed in normal pregnancies (Smith et al., 2006b). In a small study in human volunteers (Smith et al., 2006a), relaxin administered for 5 hours increased renal plasma flow by $\sim 75 \%$ and fractional sodium excretion by $\sim 25 \%$ but did not affect glomerular filtration rate (Smith et al., 2006a). The authors suggest that the volunteers may not have been volume replete, which may offset the effects of relaxin at the afferent arteriole. Alternatively, humans, in contrast to rats, are not typically in glomerular filtration equilibrium, thereby allowing dissociation of the effects of relaxin on renal plasma flow and glomerular filtration rate (Smith et al., 2006a), or the effect may take longer to develop via alteration of the filtration coefficient. For example, during chronic relaxin infusion in the scleroderma trials, the estimated glomerular filtration rate rose significantly in the treated group (Seibold et al., 2000; Khanna et al., 2009). However, the vasodilator effects of relaxin may also influence creatinine handling in the bowels, a process that is usually upregulated in states of renal insufficiency such as scleroderma or congestive heart failure. The renal effects of relaxin in humans therefore remain to be confirmed.

6. Fibrosis. The antifibrotic effects of relaxin were the first biologic effects to be recorded (Hisaw, 1926), and a number of studies have examined this property as a possible treatment of the connective tissue disease scleroderma. Relaxin was safe and well tolerated in clinical trials and effective in some patients in a phase II trial (Seibold et al., 2000) but failed to show clinical efficacy in a larger scale phase III trial (Erikson and
Unemori, 2001). Despite these disappointing findings there are many studies in animals that show a role for relaxin in controlling collagen turnover. In relaxin knockout mice, there is a progressive increase in tissue fibrosis with age in male mice that is prevented (Samuel et al., 2007) or reversed in lung (Samuel et al., 2003b), kidney (Samuel et al., 2004b), and heart (Samuel et al., 2004a) by the administration of relaxin.

In the lung, treatment with relaxin reduces expression of collagen types I and III, increases levels of MMPs, and reduces fibrosis (Unemori et al., 1996). In kidney-derived fibroblasts, relaxin inhibits profibrotic changes induced by TGF- $\beta$ by a mechanism involving the NO/guanylyl cyclase pathway with associated decreases in Smad2 phosphorylation and nuclear localization (Mookerjee et al., 2009). Relaxin has antifibrotic effects in rat renal fibrosis models, including fibrosis produced by bromoethylamine treatment (Garber et al., 2001), in an antiglomerular basement membrane model (McDonald et al., 2003) and in spontaneously hypertensive rats (Lekgabe et al., 2005). In cardiac fibroblasts, relaxin reduces collagen type I and III expression and increases MMPs (Samuel et al., 2004a). In cardiac fibrosis after chronic stimulation of $\beta$-adrenoceptors by isoprenaline (Zhang et al., 2005) or by cardiac-specific transgenic overexpression of $\beta_{2}$-adrenoceptors (Bathgate et al., 2008), relaxin treatment markedly reduced cardiac fibrosis. In streptozotocin-treated mRen-2 rats (a model for diabetic cardiomyopathy), relaxin reduced left ventricular collagen, myocardial stiffness, and diastolic dysfunction (Samuel et al., 2008), associated with a significant decrease in TIMP-1 expression and an increase in extracellular matrix-degrading MMP-13 (Samuel et al., 2008). Relaxin also has antifibrotic actions in a number of models of liver fibrosis (Bennett, 2009). However in a chronic pressure overload model in mice, relaxin was ineffective (Xu et al., 2008), possibly because cardiac RXFP1 may be downregulated in this model or the serum concentrations of relaxin are insufficient to offset the extensive fibrosis.

Relaxin acting via RXFP1 in primary fibrochondrocytes increases the expression of mRNA for MMP-9 and MMP-13 (Ahmad et al., 2012) associated with activation of PI3K, Akt, $\mathrm{PKC} \zeta$, and ERK1/2; inhibitors of these proteins blocked MMP-9 induction and the antifibrotic effects of relaxin. Relaxin-mediated increases in MMP-9 expression were also blocked by transfection of a dominant negative form of Akt or by small interfering RNA knockdown of ERK1/2, PKC $\zeta$, Elk-1, c-fos, and, to a lesser extent, $\mathrm{NF}_{\kappa} \mathrm{B}$ (Ahmad et al., 2012). This important study connects many of the known pathways of relaxin/ RXFP1 signaling to a well recognized physiologic response to relaxin and provides insights that could be useful in translating the antifibrotic effects of relaxin into a clinical setting.

The apparent discrepancy between the highly successful preclinical antifibrosis studies and the failure of 
relaxin in the phase III human scleroderma trial can be explained by an incomplete understanding of the pathophysiology of scleroderma and by the enrolment of patients in the terminal stages of the disease. It is of interest to note that the dermal fibrosis of the relaxin knockout mouse is rescued by exogenous relaxin at 6 months but not rescued by administration at 12 months of age (Samuel et al., 2005).

One of the interesting aspects that have emerged is that the antifibrotic properties of relaxin are clearly seen in disease conditions associated with excessive collagen deposition. Explanations for this unusual and potentially useful property of relaxin are now starting to emerge. In rat kidney myofibroblasts, it was discovered that the antifibrotic actions of relaxin are completely abolished by the $\mathrm{AT}_{2} \mathrm{R}$ antagonist PD123319 (Chow et al., 2014). In an in vivo fibrosis model in mice, produced by unilateral ureteric obstruction, the protective effects of relaxin are lost when the $\mathrm{AT}_{2} \mathrm{R}$ is either absent (in $\mathrm{AT}_{2} \mathrm{R}^{-/ \mathrm{y}}$ mice) or blocked by PD123319, confirming that the $\mathrm{AT}_{2} \mathrm{R}$ is obligatory for the antifibrotic actions of relaxin. These effects are not produced by relaxin interacting directly with the $\mathrm{AT}_{2} \mathrm{R}$, because the peptide does not bind to this receptor. However, it was found that RXFP1 can form constitutive heterodimers with $\mathrm{AT}_{2} \mathrm{R}$ in cell-based models, and the RXFP1-AT $\mathrm{A}_{2} \mathrm{R}$ heterodimers may mediate the downstream signaling pathways originally attributed to relaxin (Heeg et al., 2005; Mookerjee et al., 2009; Chow et al., 2012), including inhibition of the TGF- $\beta_{1} / \mathrm{pSmad} 2$ axis and reduced TGF- $\beta_{1}$-induced collagen deposition. Because $\mathrm{AT}_{2} \mathrm{Rs}$ are expressed at low levels in tissues (Matsubara, 1998; Carey, 2005; Jones et al., 2008) and fibroblasts under physiologically quiescent states but are dramatically increased in number and activity under pathologic conditions, their increased availability in injured/diseased tissues increases their functional importance (Siragy and Carey, 1997; Savoia et al., 2006). Hence, pathologic conditions create an environment in which RXFP1-AT $\mathrm{T}_{2} \mathrm{R}$ heteromerization is more likely to take place and may help explain why relaxin displays its antifibrotic effects to a pronounced extent under pathologic conditions.

Relaxin also has beneficial effects in wound healing (Casten and Boucek, 1958) that may involve the vasodilator effects mentioned above but, in addition, may involve the synthesis of new blood vessels by enhancing the local production of VEGF (Unemori et al., 2000).

7. Organ Protection. Relaxin not only protects the myocardium from ischemia-reperfusion (IR) injury (see above) but also protects other tissues. Thus, there is protection from IR injury in rat liver (Boehnert et al., $2005,2008,2009$ ) by attenuating oxidative stress, leukocyte activation, and improving oxygen supply. In kidney IR in rats, relaxin reduces renal apoptosis and $\mathrm{TNF}-\alpha$ levels as well as creatinine and urea levels (Yoshida et al., 2013). In a similar model, relaxin decreases inflammatory cytokines, counteracts oxidative damage by increasing SOD-1 and
SOD-2 expression, and ameliorates neutrophil-related injury as assessed by myeloperoxidase levels (Collino et al., 2013). Finally, the beneficial effects of relaxin are also observed in IR experiments in isolated rat lungs (Alexiou et al., 2010, 2013) where there is inhibition of leukocytes and oxidative surge, as well as prevention of ET-1 stimulation, resulting in less edema formation and lower pulmonary vascular pressure.

NO appears to be critically involved in organ protection (Masini et al., 1997; Alexiou et al., 2010, 2013; Collino et al., 2013). In rat lung, relaxin evokes an early and moderate increase in iNOS dependent on ERK1/2 activation that is counterbalanced by PI3K recruitment (Alexiou et al., 2013). In rat kidney (Collino et al., 2013), the peptide upregulates iNOS and activates eNOS via PI3K. It remains to be investigated whether over the long term, additional modes of action, such as the actions of relaxin on the GR pathway, come into play.

8. Formation and Spread of Tumors. Relaxin is expressed in endometrial (Kamat et al., 2006), mammary (Tashima et al., 1994), thyroid (Hombach-Klonisch et al., 2006), and prostate tumors (Thompson et al., 2006; Feng et al., 2007). The peptide is associated with breast cancer (Bani, 1997; Silvertown et al., 2003), and relaxin treatment of breast cancer cells implanted into nude mice increases their invasive potential (Binder et al., 2002). In contrast, longer-term application of relaxin (up to 8 days) reduces mammary xenograft growth in mice (Radestock et al., 2008). The elevated serum relaxin levels that have been reported in breast cancer patients are associated with metastatic disease (Binder et al., 2004). Relaxin is also associated with prostate cancer progression in the mouse xenograft model (Silvertown et al., 2006), and blocking the actions of relaxin or its receptor in this model decreases cancer growth (Feng et al., 2010). In patients with prostate cancer, elevated relaxin levels are linked to cancer progression, metastasis, and androgen independence (Thompson et al., 2006). Likewise, relaxin heightens the collagenolytic potency of thyroid cancer cells by upregulating MMP-2 that facilitates greater in vitro invasiveness (Bialek et al., 2011). In human osteosarcoma cells, relaxin promotes faster in vitro growth, invasion, and angiogenesis via the Akt and VEGF pathways, and small interfering RNA-mediated knockdown of relaxin greatly mitigates these effects (Ma et al., 2013a,b). The peptide hastens the proliferation of human leiomyoma cells (Suzuki et al., 2012). A clinical study in 108 patients with hepatocellular carcinoma identified expression of relaxin in tumor tissue as an independent predictor of poor prognosis (Pan et al., 2013). Similarly, high plasma relaxin levels were associated with a worse prognosis in 146 patients with esophageal squamous cell carcinoma (Ren et al., 2013). A recent study also suggests that CTRP8 interacts with RXFP1 to facilitate cell migration in brain cancer, dependent on activation of PI3K and PKC (Glogowska et al., 2013). In summary, relaxin does 
not appear to initiate cancer, but, like other growth and angiogenic hormones, it may promote cancer growth and/ or spread by its actions on enhanced matrix degradation and angiogenesis. To date, though lacking definitive proof, this might be clinically relevant for mammary, thyroid, and prostate cancer.

9. Diabetes. Although relaxin shares many structural similarities with insulin (Schwabe and McDonald, 1977), the peptides interact with different receptors and activate quite different signal transduction mechanisms. RXFP1 is a GPCR, whereas insulin receptors are tyrosine kinases and relaxin and insulin do not display crossreactivity. Although this would appear to argue against any connection between the hormones, there are studies that suggest that relaxin can influence carbohydrate metabolism. For instance, many insulin-dependent diabetic women have hypoglycemic episodes during the first trimester of pregnancy, when circulating levels of relaxin are higher, whereas increased insulin may be required during the third trimester, when relaxin concentration are lowest (O'Byrne et al., 1978). Additionally, intraperitoneal dosing of human relaxin inhibits ad libitum feeding in male rats when given during the early dark phase of the circadian clock (McGowan et al., 2010).

Relaxin concentrations in the serum of diabetic women are higher than those observed in the serum of nondiabetic women during each trimester of pregnancy (Steinetz et al., 1992; Whittaker et al., 2003). One possible explanation is that the increased relaxin is a physiologic response to the insulin-resistance that occurs during pregnancy. More circumstantial evidence comes from studies showing that circulating relaxin levels correlate positively with insulin sensitivity and inversely with $\beta$-cell function in women with type-2 diabetes mellitus (T2DM) (Szepietowska et al., 2008) and studies showing an increase in plasma relaxin after successful antidiabetic therapy in men with T2DM (Schondorf et al., 2007). The cellular mechanism is not clear, but it has been reported that relaxin increases the binding of insulin to its receptors on adipocytes (Olefsky et al., 1982; Jarrett et al., 1984) an effect that would enhance glucose uptake. In rat cardiac fibroblasts, high-glucose conditions increase the expression of endogenous relaxin (Wang et al., 2009). In human amniotic epithelial cells, relaxin enhances expression of insulin-like growth factor-2 (Millar et al., 2003). In preclinical studies in lean mice that are insulin-resistant after a high-fat diet, relaxin increases glucose uptake into skeletal muscle by improving hemodynamics and angiogenesis that is known to be an important factor in determining the response to insulin (Bonner et al., 2013). More recently, relaxin applied over 12 days in a genetic mouse model of T2DM lowered blood glucose levels, from an average of $660 \mathrm{mg} / \mathrm{dl}$ in vehicle-treated animals to 490 $\mathrm{mg} / \mathrm{dl}$ (Bitto et al., 2013). In addition, relaxin has also been shown to activate peroxisome proliferator-activated receptor- $\gamma$ and enhance the actions of the peroxisome proliferator-activated receptor- $\gamma$ activator rosiglitazone in cells expressing RXFP1 (Singh and Bennett, 2010). In this way relaxin treatment would be expected to influence insulin sensitivity.

Originally identified for its effects on the female reproductive tract, relaxin has now been recognized to have actions in the central nervous system (CNS), cardiovascular system, and kidney, on glucose metabolism and in cancer metastasis in both males and females. Although it has important and well defined roles in pregnancy and reproduction in many species, it is not mandatory for successful pregnancy in humans. The vasodilatory and antifibrotic actions of relaxin on blood vessels are the subject of intense interest, and relaxin was recently shown to produce inotropic responses in human atria. Studies of the antifibrotic actions of relaxin indicate that the effects are dependent on expression of the $\mathrm{AT}_{2} \mathrm{R}$, raising the question whether heterodimer formation between RXFP1 and other GPCRs underpins other physiologic responses to the hormone. Connections have also been established between relaxin and the formation and spread of tumors, although the mechanisms involved are less clear. Relaxin also appears to improve insulin sensitivity and improves glucose tolerance in animal models of T2DM.

\section{B. Insulin-Like Peptide 3 and Relaxin Family Peptide Receptor 2}

1. Reproduction. INSL3 is primarily a reproductive hormone and was first cloned from testicular cDNA libraries (Adham et al., 1993; Pusch et al., 1996). The peptide is secreted by testicular Leydig cells (Adham et al., 1993; Pusch et al., 1996) but is also found in human cyclic corpora lutea (Tashima et al., 1995); ruminant ovary, uterus, and placenta (Bathgate et al., 1996; Roche et al., 1996); mouse (Zimmermann et al., 1997); and marmoset ovary (Zarreh-Hoshyari-Khah et al., 1999). Importantly INSL3 is a circulating hormone in both males (Foresta et al., 2004) and females (AnandIvell et al., 2013), although the levels are much higher in males.

The best characterized physiologic role for INSL3 is in the male where it controls testis descent. Male INSL3 knockout mice show normal development but are infertile and bilaterally cryptorchid, with the testis located high in the abdominal cavity adjacent to the kidney (Nef and Parada, 1999; Zimmermann et al., 1999). A similar phenotype is displayed in RXFP2 knockout mice, confirming the cryptorchid phenotype (Overbeek et al., 2001; Gorlov et al., 2002). Thus, the INSL3/RXFP2 system appears to be essential for the development of the gubernaculum during embryogenesis and for normal transabdominal testicular descent.

Cryptorchidism is the most common birth defect of the male genitalia; affecting $1-4 \%$ of live male births, with a greater incidence in premature infants (Ivell and 
Hartung, 2003). Although the studies in INSL3 or RXFP2 knockout mice suggested that mutations in these genes could account for cryptorchidism, this is unlikely to be the case in all human infants, because few of these mutations result in a functional change in either the peptide or the receptor (Tomboc et al., 2000; Gorlov et al., 2002). Furthermore, because all mutations of INSL3 and RXFP2 identified in cryptorchid patients have been found only as heterozygotes and the expression of INSL3 in testicular Leydig cells is high, it is unlikely that a mutation in a single allele would be sufficient to induce bilateral cryptorchidism.

INSL3 is highly expressed in the testicular Leydig cells in adult mammals and is considered an important marker of Leydig cell function (Ivell and Anand-Ivell, 2009; Ivell et al., 2014). Additionally, INSL3 circulates at nanogram per milliliter levels in males and therefore has potential endocrine functions such as that on bone function. RXFP2 receptors are expressed on Leydig cells (Anand-Ivell et al., 2006) and on germ cells in the seminiferous tubules (Kawamura et al., 2004; AnandIvell et al., 2006; Filonzi et al., 2007). A recent study suggested that INSL3 influences steroid production by Leydig cells (Pathirana et al., 2012), and studies in male rats suggest that INSL3 is a paracrine factor involved in male germ cell survival (Kawamura et al., 2004). However, it should be noted that androgen production is normal in INSL3 and RXFP2 knockout mice (Nef and Parada, 1999; Zimmermann et al., 1999; Overbeek et al., 2001; Gorlov et al., 2002). Additionally, conditional deletion of RXFP2 in male germ cells resulted in normal fertility (Huang et al., 2012).

In females, INSL3 is produced in the thecal cells of the ovary and in the corpus luteum (Ivell and Bathgate, 2002). In mouse ovaries, Insl3 expression is higher in the follicular than the luteal phase (Zimmermann et al., 1997), and female INSL3 knockout mice demonstrate impaired fertility associated with longer estrous cycle length, smaller litter sizes, accelerated follicular atresia and luteolysis, and premature loss of corpora lutea (Nef and Parada, 1999; Spanel-Borowski et al., 2001). Further evidence that INSL3 is a circulating hormone of ovarian origin in females comes from the observation that postmenopausal women have undetectable plasma INSL3 levels (Ivell and Anand-Ivell, 2009). Studies in cohorts of adult women suggest that INSL3 plasma levels are indicative of the number of ovarian antral follicles recruited and growing within a follicular wave (AnandIvell et al., 2013). As INSL3 is a product of the thecal cells of ovarian follicles, plasma INSL3 levels have been investigated and shown to be elevated in polycystic ovary syndrome, a common female endocrine disorder characterized by excessive production of androgens from ovarian follicles (Gambineri et al., 2007; Szydlarska et al., 2012; Anand-Ivell et al., 2013). Importantly, a recent study in cows demonstrated that INSL3 has a positive autoregulatory role in maintaining thecal androgen production that is essential for normal ovarian follicle development (Glister et al., 2013).

2. Brain. RXFP2 expression has been found in human brain by RT-PCR (Hsu et al., 2002) and in the rat parafascicular nucleus, dorsolateral, ventrolateral, and posterior thalamic nuclei and medial habenula by in situ hybridization histochemistry (Shen et al., 2005; Sedaghat et al., 2008). These sites correspond to the distribution of RXFP2 determined by $\left[{ }^{125} \mathrm{I}\right] \mathrm{INSL} 3$ binding (Sedaghat et al., 2008). Insl3 expression has been demonstrated in the rat brain (Gundlach, personal communication) and also in the bovine hypothalamus by RT-PCR (Bathgate et al., 1996) and Northern blotting (Bathgate et al., 1999). The effects of INSL3 administration to rat brain suggest that RXFP2 influences sensorimotor function (Sedaghat et al., 2008).

3. Bone and Other Tissues. Physiologic functions of INSL3 in other tissues have been postulated based on INSL3 and RXFP2 expression patterns. In young adults with cryptorchid hypogonadism associated with the T222P mutation of RXFP2, 64\% had reduced bone density but normal plasma testosterone levels (Ferlin et al., 2008). RXFP2 mRNA and protein are present in human osteoblasts, and an osteoblast cell line responded to INSL3 with a concentration-dependent increase in cAMP. Mouse osteoblasts also express RXFP2, but not Insl3, and RXFP2 knockout mice are osteopenic and have functional osteoblast impairment (Ferlin et al., 2008). INSL3 regulates expression of genes required for proliferation and differentiation, matrix deposition, and osteoclastogenesis in cultured human osteoblasts (Pepe et al., 2009). Deficits in INSL3/RXFP2 signaling are correlated with reduced bone mass (Ferlin et al., 2008, 2009). Thus INSL3 may have a role in bone physiology, and RXFP2 mutations may be linked with osteoporosis in men (Ferlin et al., 2013). Interestingly, recent studies suggest that RXFP2 is associated with horn growth in sheep (Johnston et al., 2013) and cows (Wiedemar et al., 2014), suggesting actions of INSL3 on bone is a common feature in male mammals.

In tumor biology, there is altered expression of INSL3 and a splice variant in human hyperplastic thyroid adenoma and thyroid cancer (Hombach-Klonisch et al., 2003). INSL3 is also present in human prostate carcinomas and increases motility in the human androgen-insensitive prostate carcinoma cell line PC-3 (Klonisch et al., 2005). All human thyroid adenomas, three types of human thyroid carcinomas, and mouse noncancerous follicular epithelial cells of the thyroid express RXFP2 mRNA (HombachKlonisch et al., 2010). Thyroid cancer cells expressing INSL3 demonstrate enhanced motility and increased colony formation in vitro and enhanced tumor growth in vivo. Finally, INSL3 increases levels of the calcium binding protein S100A4, which increases cancer cell mobility and enhances tumor tissue vascularization, suggesting that S100A4 is a downstream target of RXFP2 signaling in thyroid cells (Hombach-Klonisch 
et al., 2010). Thus INSL3 and RXFP2 appear to play a role in cancer metastasis.

RXFP2 mRNA expression is highest in rat kidney at late stage gestation and decreases dramatically at birth with lowest levels in adulthood (Fu et al., 2006). RXFP2 is expressed in mesangial cells in mature glomeruli and inhibits proliferation of cultured primary glomerular cells, suggesting that INSL3 and RXFP2 influence the genesis or maturation of renal glomeruli and regulate mesangial cell density (Fu et al., 2006). Pod1 is one of the main transcription factors involved in glomerulogenesis (Quaggin et al., 1999), and an E-box consensus sequence capable of binding Pod1 in conjunction with other helix loop helix transcription factors is located upstream of the gene for RXFP2 (Funato et al., 2003). Therefore, Pod1 may regulate expression of RXFP2 in the kidney during development to facilitate glomerular cell proliferation (Familari et al., 2009). RXFP2 expression levels in the embryonic kidney were significantly greater in Pod1 knockout mice than in heterozygous or wild-type controls, indicating that RXFP2 is downstream of Pod1 and Pod1 negatively regulates expression of RXFP2 in the glomeruli (Familari et al., 2009).

Although the INSL3 and RXFP2 system has primarily been studied for its specialized roles in both male and female reproductive physiology, there is emerging evidence to suggest that this system may have other roles in the CNS, in cancer metastasis, in bone physiology and in the kidney.

\section{Relaxin-3 and Relaxin Family Peptide Receptor 3}

Relaxin-3 and RXFP3 expression is highest in the brain, and most studies examine roles in the CNS. The evidence for the receptor-ligand pair includes their coevolution (Wilkinson et al., 2005a), their coexpression in brain (Bathgate et al., 2002; Burazin et al., 2002; Liu et al., 2003b; Tanaka et al., 2005; Ma et al., 2009b,c; Smith et al., 2010, 2011), and the high affinity of relaxin-3 for RXFP3 (Liu et al., 2003b; van der Westhuizen et al., 2007, 2010). Although relaxin-3 may interact with RXFP1 (Sudo et al., 2003) and RXFP4 (Liu et al., 2003a) and possibly RXFP2 in some species (Scott et al., 2005a), these interactions are probably not important physiologically because the expression pattern of relaxin-3 suggests that it would be unlikely to interact with the other RXFP receptors.

1. Stress. Relaxin-3 and RXFP3 are present in hypothalamic and extrahypothalamic regions involved in the hypothalamic-pituitary-adrenal axis (Sutton et al., 2004; Liu et al., 2005a; Ma et al., 2007a; Smith et al., 2010). Corticotropin releasing factor (CRF) is synthesized and released from the paraventricular nucleus of the hypothalamus (PVN) in response to stress. RXFP3 is highly expressed in the PVN, as well as other regions associated with stress and anxiety, such as the bed nucleus of the stria terminalis, lateral septum, periaqueductal gray, and dorsal raphe (Liu et al., 2005a). Neural responses to CRF are mediated by the $\mathrm{CRF}_{1}$ receptor that is expressed in most relaxin-3-containing neurons in the NI (Sutton et al., 1982; Tanaka et al., 2005). Administration of CRF intracerebroventricularly to rats caused activation of relaxin-3-containing neurons, and neurogenic stressors increased relaxin-3 mRNA in the NI (Tanaka et al., 2005), where relaxin-3 is found in presynaptic vesicles. Relaxin-3 mRNA in the rat NI is increased after a forced swim stress paradigm (Banerjee et al., 2010), and these effects are partly blocked by pretreatment with the $\mathrm{CRF}_{1}$ antagonist antalarmin. Although the precise CRF neuronal projection(s) to the NI relaxin-3 cells have not yet been described, hypothalamic and preoptic areas containing CRF neurons have been shown to project to the NI (Goto et al., 2001). CRF levels in rat, monkey, or human CSF are in the picomolar range even in pathologic states (Garrick et al., 1987; Arborelius et al., 1999). Altered relaxin-3 expression in the NI due to stress is therefore unlikely to result from CRF in the spinal fluid and most likely comes from CRF neuronal projection(s). Ascending projections from the NI (Olucha-Bordonau et al., 2003) are consistent with RXFP3 receptor autoradiography (Sutton et al., 2004) and with involvement of relaxin-3/RXFP3 in neuropsychiatric disease (Smith et al., 2010).

2. Feeding and Metabolism. In rodents, RXFP3 is expressed in the PVN and supraoptic nucleus (Smith et al., 2010, 2011), suggesting a role in metabolic control. Injection of relaxin-3 intracerebroventricularly to rats either in the early light or early dark phase, transiently increased food intake (McGowan et al., 2005). This stimulatory effect did not result from increased spontaneous activity or arousal and is likely mediated by RXFP3 and not RXFP1, because human relaxin administration had no effect when given during the early light phase (McGowan et al., 2005) or decreased feeding when given during the early dark phase (McGowan et al., 2010) in ad libitum-fed rats. The inhibitory feeding response to human relaxin when administered in the dark as opposed to the light phase suggests a circadian influence involving RXFP1 that is also expressed in hypothalamic centers associated with feeding (Ma et al., 2006).

Both acute and chronic relaxin-3 infusions into the PVN increased food intake (McGowan et al., 2006). Injection into the supraoptic nucleus, arcuate nucleus, and anterior preoptic area also transiently increased food intake. However, it is unclear how relaxin-3 exerts its effects in the arcuate nucleus or anterior preoptic area, because RXFP3 is not present in these nuclei. Finally, intracerebroventricular infusion of the selective RXFP3 agonist R3/I5 also increased food intake, an effect blocked by preadministration of the antagonist R3 (B $\Delta 23-27) R / I 5$ (Kuei et al., 2007). Administration of R3 (B $\Delta 23-27) R / I 5$ intracerebroventricularly alone has no effect on food intake (Sutton et al., 2009), suggesting that under resting conditions there is minimal tone in the relaxin-3/RXFP3 system. 
Although relaxin-3 influences food intake, effects on body weight are equivocal. In two studies, chronic intracerebroventricular relaxin-3 increased body weight (Hida et al., 2006; Sutton et al., 2009), but others show no weight change after intracerebroventricular or intrahypothalamic injection of relaxin-3 (McGowan et al., 2005, 2006). In rats, chronic intracerebroventricular administration of the antagonist R3(B $\Delta 23-27) \mathrm{R} / \mathrm{I} 5$ did not affect body weight (Sutton et al., 2009). There is also little evidence to suggest that body weight is influenced by endogenous relaxin-3. Although relaxin-3 knockout mice were initially reported to have lower body weights, the first study (Sutton et al., 2009) was performed on a mixed 129:B6 mouse strain before the mice were backcrossed, and the differential phenotype could not be reproduced on a C57/B16N background. This dependence of phenotype on strain suggests there may be compensation for a chronic absence of relaxin-3 by other systems.

Several studies investigating the effects of relaxin-3 infusion on feeding also measured blood hormone levels. In rats, chronic infusion of RXFP3 agonist (relaxin-3 or R3/I5) intracerebroventricularly increased plasma leptin, insulin (Hida et al., 2006), adiponectin, testosterone, angiotensinogen, and decreased growth hormone levels (Sutton et al., 2009). Administration into the PVN caused increases in leptin in ad libitum-fed animals and in thyroid-stimulating hormone in ad libitum- and pair-fed animals (McGowan et al., 2006). Intra-PVN agonist dosing increased feeding but failed to significantly change other behaviors such as drinking, grooming, burrowing, rearing, general locomotion, apparent sleep, head down, or tremor (McGowan et al., 2005). Similarly, chronic R3(B $\Delta 23-27) R / I 5$ dosing seemed only to decrease plasma levels of growth hormone (Sutton et al., 2009) while not altering the other hormones measured. These effects occurred in the absence of changes in energy expenditure.

Studies of the metabolic role of relaxin-3 have provided only limited supporting mechanisms, although considerable evidence supports the actions of the peptide on feeding behavior. Although there is RXFP3 expression in the pancreas and in vitro relaxin-3 suppression of insulin secretion has been reported in isolated tissue (Yamamoto et al., 2009), human plasma relaxin-3 levels are in the picograms per milliliter (low picomolar) range and do not vary in the diabetic state (Zhang et al., 2013). Considering relaxin-3/RXFP3 expression occurs in CNS areas associated with neuroendocrine and behavioral control (Sutton et al., 2004; Ma et al., 2009a; Ganella et al., 2013b), metabolic effects of an RXFP3 agonist may be largely secondary to increased feeding behavior. As noted above, central injection of an RXFP3 agonist in rats increased feeding both acutely (McGowan et al., 2005) and chronically (Hida et al., 2006; Sutton et al., 2009), suggesting a lack of tolerance. In a second rat model, chronic viral expression of an RXFP3 agonist achieved similar results (Ganella et al., 2013a,b). Acute or chronic dosing of an antagonist alone to rats was ineffective (Sutton et al., 2009), suggesting that relaxin-3 tone is low under resting physiologic conditions. Conversely in mice, RXFP3 agonist dosing does not increase feeding under resting conditions (Ganella et al., 2013b). A recent study showed that an RXFP3 antagonist, although ineffective in altering feeding in baseline/sated mice, blocked motivated feeding behavior (Smith et al., 2014). In this study, R3(B1-22)R/I5 blocked food anticipatory activity after a 4-hour food restriction when administered intracerebroventricularly to C57/B16J control mice but not to congenic relaxin-3 knockout mice. Antagonist treatment also significantly reduced feeding in mice trained to expect palatable food at a given time. Although relaxin-3 and RXFP3 neuroanatomy is consistent with effects on feeding and body weight in monkeys (Ma et al., 2009b,c), more studies are needed to predict translation of these effects to humans. In addition, detailed studies are also needed to tease out potential confounding effects on reward, motivation and arousal (see below) and to determine whether there is a true feeding response to relaxin-3 stimulation.

3. Behavioral Activation and Arousal. Evidence for a role for the relaxin-3/RXFP3 system in behavioral activation and arousal is derived from studies that examined colocalization of projections of relaxin-3 neurons from the NI to septal GABAergic and cholinergic neurons that project to the hippocampus (Ma et al., 2009a). The rodent septohippocampal pathway is heavily innervated by relaxin-3 positive projections from the NI. This pathway generates the hippocampal theta rhythm, with oscillations at $4-12 \mathrm{~Hz}$ that is controlled by pacemaker neurons of the medial septum (MS). The hippocampal theta rhythm is involved in behaviors such as vigilance, exploration, orientation, navigation, locomotor control, and working memory. Electrical stimulation of the NI causes theta rhythm in the hippocampus and lesions of the NI disrupt theta rhythm initiated by stimulation of the reticularis pontine oralis (Nunez et al., 2006). In both anesthetized and conscious rats, RXFP3 modulates neuronal activity in the hippocampus and MS to promote hippocampal theta rhythm (Ma et al., 2009a) and blockade of RXFP3 in the MS with the antagonist R3 (B $\Delta 23-27) R / I 5$ dose dependently impairs performance in a paradigm investigating theta rhythm-dependent spatial working memory, effects that are reversed by coadministration of the RXFP3 selective agonist R3/I5 (Ma et al., 2009a).

The relaxin-3/RXFP3 system also influences locomotor activity in rodents. Although chronic intracerebroventricular relaxin-3 had no effect on locomotor activity in male Wistar rats (Hida et al., 2006), acute injection of the selective RXFP3 agonist R3/I5 increased locomotor activity, whereas the antagonist R3(B $\Delta 23-27) \mathrm{R} / \mathrm{I} 5$ given alone had no effect (Sutton et al., 2009). Effects of the RXFP3 agonist on general locomotor activity, although statistically significant in one study (Sutton et al., 2009), seem to be small in magnitude and were not reproduced after intraPVN injections (McGowan et al., 2005). Interestingly, 
female relaxin-3 knockout mice were hypoactive relative to wild-type littermates in several paradigms including the locomotor cell, large open field, Y-maze, and novel object tests (Smith et al., 2009; Hosken et al., 2014). A similar phenotype is displayed in mice receiving the RXFP3 antagonist R3(B1-22)R/I5 or R3(B1-22)R (Smith et al., 2014).

Because serotonin (5-hydroxytryptamine [5-HT]) has well established roles in cognitive, emotional, and behavioral control (reviewed in Cools et al., 2008) and the $\mathrm{NI}$ is located close to the dorsal raphe, a region enriched in 5-HT neurons, studies were carried out on the effects of 5-HT on relaxin-3 expression (Miyamoto et al., 2008); most relaxin-3-containing neurons of the NI were found to coexpress $5-\mathrm{HT}_{1 \mathrm{~A}}$ receptors. Inhibition of 5-HT synthesis for 3 days increased relaxin-3 mRNA in the NI. The correlation between $5-\mathrm{HT} / 5-\mathrm{HT}_{1 \mathrm{~A}}$ inactivity (because of 5-HT depletion) and altered relaxin-3 expression in the NI suggests a role for these systems in anxiety and/or depression. Recent studies show anxiolytic and antidepressant effects of intracerebroventricular RXFP3 agonist dosing in behavioral rat models (Ryan et al., 2013a). These studies emphasize the need to determine the relationship between 5 -HT and relaxin-3/RXFP3 systems.

Recent studies in rats link RXFP3 circuits to ethanol self-administration and reinstatement behaviors (Ryan et al., 2013b). The R3(B $223-27) R / 15$ or R3(B1-22)R RXFP3 antagonists administered intracerebroventricularly reduced alcohol self-administration without concomitant alterations in feeding, locomotor behaviors, or memory. The effects on alcohol self-administration were specific, because the antagonists had no effect on sucrose selfadministration. In addition, RXFP3 antagonism blocked both cue and stress-induced reinstatement of alcohol seeking. The reduction of alcohol self-administration/ reinstatement was linked to the stress-responsive bed nucleus of the stria terminalis, because direct injection of the RXFP3 agonists into the bed nucleus of the stria terminalis also reduced alcohol self-administration.

Extensive brain mapping and behavioral studies have established that the relaxin-3 and RXFP3 system is involved in stress, metabolic control, and behavioral activation and arousal. Recent studies suggest that modulation of the system could be useful in the treatment of forms of addiction.

\section{Insulin-Like Peptide 5 and Relaxin Family Peptide Receptor 4}

Although RXFP4 was originally identified as a receptor for relaxin-3 (Liu et al., 2003a), the dramatically different expression patterns of ligand and receptor suggested that relaxin-3 was not the preferred cognate ligand. RXFP4 mRNA is present in a variety of human tissues including brain, kidney, testis, thymus, placenta, prostate, salivary gland, thyroid, and colon (Liu et al., 2005b; Burnicka-Turek et al., 2012; Mashima et al.,
2013; Thanasupawat et al., 2013). Many of these tissues also express INSL5 (Burnicka-Turek et al., 2012; Mashima et al., 2013; Thanasupawat et al., 2013), and both RXFP4 and Insl5 are pseudogenes in rats and dogs (Chen et al., 2005; Wilkinson et al., 2005a). INSL5 was shown to be a high-affinity ligand for RXFP4 (Liu et al., 2005b), and thus the receptor-ligand coevolution, pharmacology, and similarities in expression profiles strongly indicate that RXFP4 is the endogenous receptor for INSL5.

1. Metabolism. There is considerable interest in GPCRs that are located in pancreatic islet $\beta$-cells and represent potential targets for the treatment of T2DM (Ahren, 2009). One of the most studied systems is the incretin hormone system: glucagon-like peptide 1 (GLP-1) and its receptor GLP-1R. GLP-1 is secreted from enteroendocrine-L cells that are located in the gastrointestinal tract and concentrated in the colon and rectum. The peptide acts on GLP-1R located in a number of tissues including the GI tract, heart, kidney, lung, pancreatic $\beta$-cells, and brain. GLP-1 increases insulin release from the pancreas and improves glucose tolerance (Ahren, 2009). The INSL5 and RXFP4 system displays similarities with the incretins. Like GLP-1, INSL5 is concentrated and released from enteroendocrine-L cells (Grosse et al., 2014) into the circulation to act on RXFP4 located in a number of other tissues. In the mouse, RXFP4 expression has been detected by Western blotting in hypothalamus, pituitary, testis, epididymis, ovary, uterus, pancreas, and liver, with immunohistochemical localization to pancreatic islets, anterior pituitary, and Leydig cells in the testis (Burnicka-Turek et al., 2012). In humans, using a similar approach, RXFP4 was detected in heart, placenta, skeletal muscle, and pancreas (Mashima et al., 2013). Most interestingly, the development of the Insl5 knockout mouse showed that these animals have impaired glucose homeostasis. Glucose levels increase with age, and in Insl5 $5^{-1-}$ mice older than 6 months, glucose levels are significantly greater than age-matched control littermates. Glucose tolerance tests revealed impaired glucose tolerance in Insl5 ${ }^{-1-}$ mice that does not appear to be associated with changes in insulin sensitivity. The knockouts also had reduced pancreatic islet area compared with controls, which was associated with a reduced number of $\beta$-cells and lower circulating insulin levels. However there was no change in circulating GLP-1 levels (Burnicka-Turek et al., 2012). Insl5 ${ }^{-1-}$ mice also displayed impaired fertility due to a reduction in sperm motility and alterations in the estrus cycle (BurnickaTurek et al., 2012). Interestingly, recent association studies in humans suggest that polymorphisms of RXFP4 are associated with a high body mass index and show a trend to association with obesity (Munro et al., 2012). Studies in mice show an increase in food intake in wild-type mice after intraperitoneal injection of INSL5 that is not seen in RXFP4 knockout mice (Grosse et al., 2014). Plasma INSL5 levels increase with fasting or calorie restriction but are lowered by feeding adding weight to the suggestion that 
INSL5 is an orixigenic hormone released from the enteroendocrine-L cells in the gut (Grosse et al., 2014). At present, little is known of the potential mechanisms used by INSL5 and RXFP4 to produce metabolic effects, and a number of apparent anomalies will have to be addressed. For instance, to date all $\beta$-islet cell GPCRs that increase insulin secretion are either $\mathrm{G} \alpha_{\mathrm{s}}$ or $\mathrm{G} \alpha_{\mathrm{q}}$ coupled, and $\mathrm{G} \alpha_{\mathrm{i}}$-coupled receptors actually reduce insulin secretion (Ahren, 2009). This appears to conflict with current evidence suggesting that RXFP4 is $\mathrm{G} \alpha_{\mathrm{i}}$-coupled (Liu et al., 2003a, 2005a), although very little work has focused on RXFP4 and no studies have directly examined receptor function in pancreatic islets.

The emergence of recent evidence that INSL5 is an incretin secreted from the enteroendocrine-L cells and that both INSL5 and RXFP4 knockout mice display impaired glucose tolerance has awakened considerable interest in this ligand/receptor pair. However, more detailed biologic studies are required to firmly establish its role in metabolic control processes.

\section{Unresolved Pharmacological and Therapeutic Issues and Future Directions}

Although there have been significant advances in our understanding of the physiologic roles of relaxin family peptides and their receptor systems in recent years, there are still significant knowledge gaps. There is increasing recognition of the complexity of relaxin signaling, with recent studies suggesting that some of the pleiotropic actions of relaxin may be explained by the formation of heterodimer complexes with other GPCRs. Although this may unveil new opportunities for therapeutic intervention it may also explain why relaxin has failed in several clinical trials despite a promising preclinical profile. Clearly if heterodimer complexes are formed and are functional in pathologic conditions and blockade of the partner receptor can prevent the actions of relaxin, then a thorough understanding of the condition and treatments being received by the patient will be necessary to obtain the required therapeutic outcome. Significant new tools have emerged for the study of RXFP receptors, including small molecule allosteric agonists at RXFP1 and refined peptide agonists and antagonists at all RXFP receptors, together with a better understanding of the structure-activity relationships of the peptides and their receptors. However much remains to be done to identify the signaling patterns associated with the new molecules and how these properties can be used therapeutically. Likewise, although we now know a great deal more about the functional domains of the RXFP1 LDLa module, it is not yet apparent how it mediates signaling in response to relaxin. It is possible that it constitutes a tethered ligand, but definitive evidence has yet to be obtained.

A long-standing unresolved issue is whether there are physiologically relevant levels of relaxin circulating in nonpregnant women or in men. As discussed previously (see section V.A.1), relaxin is produced in the human ovary during the luteal phase of the menstrual cycle and can be measured in the circulation at this time (Eddie et al., 1990b; Stewart et al., 1990; Chen et al., 2003). Levels at other stages of the cycle are very low, and there is no evidence of circulating relaxin in postmenopausal women. It is still contentious as to whether there are physiologically relevant levels of circulating relaxin in men. Various studies have examined plasma relaxin levels in both male and female patients with cardiovascular disease with varied results (see section V.A.4). One problem with these studies is the use of numerous different immunoassays, most of which are incompletely characterized and the fact that relaxin levels in female patients have never been correlated with reproductive status ( $\mathrm{Du}$ et al., 2010). However it should also be noted that relaxin is produced locally in many tissues, and it is possible that many actions of relaxin are associated with paracrine production rather than circulating relaxin in nonpregnant women and men. It is also feasible that the normally low circulating relaxin concentrations that are difficult to measure by current methods do have a physiologic function. The recent evidence for relaxin signaling at highly sensitive RXFP1 signalosomes that respond to subpicomolar concentrations of relaxin provides a basis for responses to circulating peptide although their physiologic function is still unclear.

\section{A. Signaling Paradigms}

1. Heterodimerization of Relaxin Family Peptide Receptor 1: A Possible Explanation for the Selective Actions of Relaxin in Diseased or Pathologic States? Although there is evidence that RXFP1 and RXFP2 form homo- and heterodimers in cell lines (Kern et al., 2008; Svendsen et al., 2008a,b), a recent study demonstrated that the antifibrotic effects of relaxin in kidney are dependent on heterodimerization between RXFP1 and the angiotensin $\mathrm{AT}_{2} \mathrm{R}$ (see section III.A.4; Chow et al., 2014). The inhibitory effects of relaxin on excess collagen deposition were completely lost in mice treated with the $\mathrm{AT}_{2} \mathrm{R}$ antagonist PD123319 or in $\mathrm{AT}_{2} \mathrm{R}$ knockout mice, and by using BRET in cell studies, the two receptors were found to constitutively associate. These findings are particularly interesting, because they provide an explanation for the established observation that the antifibrotic effects of relaxin are seen only in pathologic conditions, with no effect on healthy tissue. Under normal conditions, $\mathrm{AT}_{2} \mathrm{R}$ are typically expressed at very low levels, but their expression dramatically increases after injury or disease (Matsubara, 1998; Carey, 2005). RXFP1 is also expressed at extremely low levels at the cell surface, with much higher levels intracellularly within the ER (Kern et al., 2007). Because many GPCRs require dimerization for trafficking from the ER to the cell surface (Milligan, 2009), the injury-induced increase in $\mathrm{AT}_{2} \mathrm{R}$ expression may result in an associated enhanced trafficking of RXFP1 (as an 
$\mathrm{AT}_{2} \mathrm{R}-\mathrm{RXFP} 1$ heterodimer) to the cell surface, thus explaining why functional effects of relaxin treatment only occur under specific pathologic conditions.

The relevance of RXFP1 heterodimerization-with the $\mathrm{AT}_{2} \mathrm{R}$ and perhaps other GPCRs-in many of the other physiologic roles of relaxin remains to be determined. This may be a general requirement for relaxin/ RXFP1 function, given the wide and pleiotropic effects of relaxin compared with the relatively limited and variable phenotype of relaxin and RXFP1 knockout and transgenic mice (Zhao et al., 1999, 2000; Samuel et al., 2003a, b, 2004b; Kamat et al., 2004; Krajnc-Franken et al., 2004; Feng et al., 2006; Ganesan et al., 2009). IUPHAR requires two of the following three conditions to be met to definitively determine the existence of heterodimers in native tissue: 1 ) evidence for physical association in native tissues or primary cells; 2) a specific functional property for the heterodimeric receptor in native tissue; and 3) use of knockout animals or RNAi to demonstrate heterodimers in vivo (Pin et al., 2007). Although conditions 2 and 3 were demonstrated for the RXFP1-AT $2 \mathrm{R}$ interaction, questions still remain regarding the interaction between RXFP1 and $\mathrm{AT}_{2} \mathrm{R}$, including a direct demonstration of receptor interaction in endogenous expression systems, whether RXFP1 usually exists as a monomer and only dimerizes with the $\mathrm{AT}_{2} \mathrm{R}$ under pathologic conditions and whether this new knowledge can be exploited to specifically target RXFP1 in disease states.

2. The Development of ML290, a Small-MolecularWeight Allosteric Agonist at Relaxin Family Peptide Receptor 1. Relaxin binding to and activation of its receptor RXFP1 involves a number of complex interactions and requires many essential receptor regions from the N-terminal LDLa module to the extracellular LRR and TM loops to the full-length C-terminal tail (see section I.B.1). As such, the development of novel molecules that mimic the actions of relaxin at RXFP1 has been a difficult challenge over a number of decades. However, the recent description of a small molecule agonist for RXFP1, ML290 (Xiao et al., 2013), has reignited the search for small molecule RXFP1 ligands.

Based on the size discrepancy between relaxin and ML290 (6000 versus $500 \mathrm{kDa}$ ) and some preliminary studies, it was considered unlikely that ML290 bound to the same regions of RXFP1 as the cognate ligand. ML290 also displayed species-specific actions, and studies of chimeras of mouse and human RXFP1 showed that ML290 binds to a region in ECL3 (Xiao et al., 2013) (see section II.A.3). Binding to a topographically distinct region of the receptor suggests a number of exciting and unique attributes for small molecule activators of RXFP1. First, although there was no evidence for signal bias by native relaxin peptides or peptide-based derivatives at RXFP1 (M. Kocan, C. Siwek, et al., personal communication), small molecule ligands binding to a distinct receptor region are likely to engender unique receptor conformations distinct from those induced by relaxin. A common corollary of this is that the signaling profile of ML290 is likely to be biased relative to relaxin. Second, binding of a small molecule to a site topographically distinct from the orthosteric (relaxin) binding sites, suggests an allosteric mode of action. Allosteric modulation of endogenous ligands has been widely documented for many other GPCRs (reviewed in Christopoulos, 2002, and May et al., 2007). Allosteric ligands are considered highly attractive as therapeutics, because of their ability to fine tune the spatial and temporal subtleties of endogenous ligand-receptor interactions (as both allosteric and orthosteric ligands can be simultaneously bound) and their potential for increased receptor selectivity (as these sites have not typically evolved to accommodate a common endogenous ligand). The potential for fine tuning of the relaxin response by a positive allosteric modulator would be highly beneficial in pathologic fibrosis, whereas a negative allosteric modulator could be beneficial for the prevention of cancer metastases. Finally, although the synthesis of peptide derivatives of relaxin has not yet produced a neutral antagonist, the discovery of a series of small agonist molecules suggests that the development of antagonists for RXFP1 may be possible in the future (because of the complicated activation mechanism of RXFP1, agonists are much more difficult to design than simple blocking/antagonist compounds). Not only are these exciting possibilities likely to result in useful experimental tools to accelerate our understanding of RXFP1 function, they will also provide novel avenues for the future design of diseasespecific RXFP1 therapeutics.

3. Novel Activators of RXFP Receptors. Over the last few years, a number of novel RXFP ligands have been described, ranging from small molecule or novel peptide activators of RXFP1 to a synthetic biased antagonist and positive allosteric modulator for RXFP3.

A recent study identified a novel protein in brain tumors that can interact with RXFP1 and promote cell invasion in the absence of relaxin (Glogowska et al., 2013). By using primary glioblastomas isolated from human patients, tumor cell migration and invasion in response to CTRP8 was found to be dependent on RXFP1 expression and involved activation of PI3K and PKC. Subsequent experiments showed an interaction between CTRP8 and RXFP1 after coimmunoprecipitation of tagged proteins coexpressed in HEK293 cells. Molecular modeling suggested that CTRP8 may interact with LRR7 and LRR8 of the RXFP1 extracellular domain, although this was not validated experimentally. This study raises some intriguing possibilities that yet again expand the repertoire of this pleiotropic receptor. First, the ability of additional peptides to bind to and activate RXFP1 may provide a further explanation (in addition to RXFP1signalosomes responding to very low concentrations of relaxin, see section III.A.3) for the surprisingly widespread effects of RXFP1 activation relative to the limited sites of relaxin production. If nonrelaxin peptides can 
activate the receptor in different cells, tissues, or pathologies, this also suggests a potential endogenous signaling bias for RXFP1 and may illuminate the pleiotropic effects of receptor activation. Second, and in contrast to the cognate ligand relaxin that requires binding to both the extracellular and TM domains of RXFP1, CTRP8 and ML290 together may provide examples of alternate mechanisms for RXFP1 activation by interacting with a single receptor domain. Although these observations are inherently intriguing, they must still be considered with caution. In both cases, there has been no direct demonstration of receptor binding using traditional radioligand binding paradigms or demonstration of interactions in isolated protein systems, and in the case of CTRP8, no RXFP1 mutagenesis to determine precise sites of receptor binding. Until these basic parameters of ligand-receptor interactions are demonstrated, it is impossible to exclude that the RXFP1-dependent functional effects might occur via interactions with a third protein or even a higher order protein complex.

A number of peptides with unique properties at RXFP3 and RXFP4 have been synthesized and represent powerful scientific tools for the study of this receptor subgroup. A positive allosteric modulator of RXFP3, 135PAM1, was recently described that showed a probe dependence for the C-terminal amidation state of the peptide agonist (Alvarez-Jaimes et al., 2012). Thus, although 135PAM1 positively modulated the cAMP inhibition stimulated by relaxin- $3_{\mathrm{NH} 2}$ and the selective agonist R3I5 $5_{\mathrm{NH} 2}$ (as determined using a CRE reporter gene), there was no effect of the modulator in combination with nonamidated peptides. A previously described antagonist for RXFP3, R3(B $\Delta 23-27) \mathrm{R} / \mathrm{I} 5$ was recently shown to have agonist properties at some signaling pathways (Kocan et al., 2014). Although the peptide antagonized some pathways downstream of relaxin or relaxin-3 activated RXFP3, it did not block others and activated SRE-mediated gene transcription itself. Such biased efficacy of so-called "antagonists" is an increasingly well-documented phenomenon for many GPCRs (Evans et al., 2010). Finally, a minimized INSL5 variant was recently synthesized (Belgi et al., 2013) that contains only the two interchain disulfide bonds that are characteristic of the relaxin family of peptides, with the intra-A-chain disulfide bond and eight residues from the $\mathrm{N}$ terminus of the A-chain removed. The minimized INSL5 analogs exhibited higher affinity and efficacy than native INSL5 and are considerably easier to synthesize. This represents an important progression for the INSL5/ RXFP4 pairing and should precipitate more studies on this hitherto neglected RXFP receptor.

There have been significant advances in recent years in both our understanding and in the development of tools for the study of signaling pathways activated by relaxin family peptides. The pleiotropic actions of relaxin are now beginning to be understood thanks to the recognition that RXFP1 is able to promote exquisitely sensitive signaling platforms or signalosomes and that it can form both homo- and heterodimers that have distinct signaling properties. The development of RXFP1 antagonists and small molecular weight allosteric agonists provides novel tools for elucidating the roles of this receptor in physiologic and pathologic conditions. RXFP2 and its cognate ligand INSL3, although structurally closely resembling RXFP1 and relaxin, appear to have relatively few and highly specialized functions. The role of relaxin-3 and RXFP3 as a neuropeptide-receptor pairing is now quite well understood, and there are examples appearing of potent and selective antagonists that promise to be useful tools. The identification of ligand bias at RXFP3 is likely of little physiologic importance owing to the distinct localization of relaxin peptides and receptors in vivo but may be a factor to be borne in mind when potential therapeutics are developed. The recognition of INSL5 as an incretin and the INSL5/RXFP4 system as players in metabolic control has stimulated increased interest in the biology of this, until now, relatively little studied receptor-ligand pair.

\section{B. Therapeutic Applications}

1. Is There Therapeutic Potential in Small-Molecule Targeting of Relaxin Family Peptide Receptor 1 Heterodimers? The recent developments showing the importance of RXFP1 heterodimerization and the identification of the small molecule agonist ML290 (see above) may provide some exciting and unique opportunities to therapeutically target RXFP1 with minimal side effects. The therapeutic potential of many pharmaceutical drugs is often limited by the development of toxic effects that can result from drug binding to nonspecific sites or drug-target binding in nontherapeutically relevant tissues. If heterodimerization of RXFP1 with the $\mathrm{AT}_{2} \mathrm{R}$ (and perhaps other GPCRs) occurs exclusively at sites of fibrotic injury, small molecule compounds that specifically target the RXFP1$\mathrm{AT}_{2} \mathrm{R}$ would be expected to exhibit exquisite selectivity, resulting in activation of the therapeutically relevant receptor to produce an antifibrotic effect only at the site of injury. Furthermore, this scenario has the capacity to incorporate endogenous temporal control of therapeutic action - thus reversal of the injury would correspondingly decrease the amount of RXFP1- $\mathrm{AT}_{2} \mathrm{R}$ heterodimer and thus the available sites of action for the small molecule. In this way, high-resolution knowledge of a system has the potential to translate into a precise spatial and temporal control of drug action at a site of injury. Whether other GPCRs heterodimerize with RXFP1 under particular injury or disease conditions and at specific locations and whether these dimers can be specifically targeted using small molecules remain to be seen.

2. Relaxin Family Peptide Receptor 3 as a Drug Target. Studies in rodents suggest that RXFP3 modulators have potential for the treatment of emotional disorders (depression and/or anxiety), social behavior deficits (autism), and inappropriate food/alcohol seeking (Smith et al., 
2011). In vivo studies show a link between relaxin-3/RXFP3 signaling and anxiety/depression (Banerjee et al., 2010; Ryan et al., 2013a); alcohol seeking/reinstatement (Ryan et al., 2013b) (the reinstatement portion of which could be partially related to stress; Weiss et al., 2001); and memory and hippocampal theta emissions (Ma et al., 2009a). The neuroanatomy of relaxin-3/RXFP3 expression is consistent with a role in these disease states and is conserved from the mouse (Smith et al., 2010) to the macaque (Ma et al., 2009b), suggesting at least some of the effects demonstrated in lower species disease models may translate to humans.

In vivo studies suggest that relaxin-3 neurons, which largely also express GABA (Ma et al., 2007a), provide predominantly inhibitory inputs. However, the RXFP3 agonist R3/I5 shows diverse effects in cells of the intergeniculate leaflet, with both depolarization and hyperpolarization recorded in different cells from the same preparation (Blasiak et al., 2013). Thus the phenotype of the relaxin-3 recipient neuron and its connections could be crucial to the mode of RXFP3 signaling. Additional work is needed to understand the pharmacology of RXFP3 and its potential therapeutic use.

The in vivo pharmacological studies performed so far have administered peptide modulators or viral constructs intracerebroventricularly or directly into CNS regions. Because this involves a labor-intensive surgical approach, RXFP3 agonists/antagonists with CNS bioavailability would greatly facilitate the extension of these studies. Unfortunately these are currently unavailable, and the positive allosteric modulator (135PAM1) that has been described displays probe selectivity precluding its use in native mammalian systems (Alvarez-Jaimes et al., 2012). Perhaps additional screening will identify useful pharmacological tools, allowing peripheral or enteric dosing of RXFP3 agonists and antagonists.

3. Relaxin Family Peptide Receptors and Metabolism. Recent evidence supports roles for relaxin family peptides in controlling metabolism. Relaxin infusion improves skeletal muscle perfusion and glucose uptake in lean mice. The effect is absent in mice fed a high-fat diet for 13 weeks (Bonner et al., 2013) but can be rescued by chronic infusion of relaxin for 3 weeks. The effect does not result from a direct action on myocytes but involves improvements in blood flow and antifibrotic actions of relaxin (Bonner et al., 2013). This may represent a novel approach to the treatment of insulin resistance.

Increased serum relaxin-3 levels have been described in women with metabolic syndrome (Ghattas et al., 2013), and it has been suggested that this may be related to the known effects of relaxin-3 on food intake (McGowan et al., 2005) and body weight (Hida et al., 2006). However, it is possible that relaxin-3 could also activate RXFP4, and it is interesting to note that there is also an increased body mass index in individuals with a polymorphism of RXFP4 (rs11264422; Munro et al., 2012). There is now strong evidence emerging that INSL5 is an incretin that acts on RXFP4 to have an orexigenic effect (Grosse et al., 2014) (see section V.D.1). INSL5 mice have impaired glucose tolerance (Burnicka-Turek et al., 2012), and there is a report that RXFP4 knockout mice have similar phenotype (Takeda patent PCT/GB2008/003023). RXFP4 may be a promising target for the treatment of obesity and T2DM.

In conclusion, there have been a number of attempts to establish relaxin as a therapeutic for the promotion of cervical ripening, facilitation of implantation, prevention of preeclampsia, and for the treatment of scleroderma. Only recently has this been successful, and relaxin is now in extended phase III clinical trials with redefined endpoints for the treatment of acute heart failure. The relative lack of success of relaxin in previous trials may be illuminated by the recent discovery that the relaxin receptor RXFP1 may in some situations act as a heterodimer partner receptor. As is the case with the RXFP1/ $\mathrm{AT}_{2} \mathrm{R}$, the expression pattern and actions of the heterodimer may be highly localized and also susceptible to blockade by antagonists of the partner receptor. This work needs to be validated in models of human disease in human tissues but could explain the variability of response observed throughout the patient cohort in previous trials. Relaxin also consistently reduces blood pressure, and antagonists may have some potential in reducing the invasiveness of cancers. The relaxin-3 and RXFP3 system has been examined as a potential target for the treatment of metabolic diseases such as anorexia, cachexia, and obesity. However, inconsistent evidence from animal studies suggests that the complex regulation of energy homeostasis by the CNS, in addition to the redundancy of these pathways, is difficult to override by manipulation of a single system. Recent studies also imply that RXFP3 may be a target for the treatment of addiction. Although still at an early stage, the studies of the biologic role of the INSL5-RXFP4 system suggest that it could have some potential for the treatment of diabetes. Small molecule agonists and antagonists of both RXFP3 and RXFP4 would facilitate more in-depth studies of the biologic roles and therapeutic potential of these two receptors.

Authorship Contributions

Wrote or contributed to the writing of the manuscript: Halls, Bathgate, Sutton, Dschietzig, Summers.

\section{References}

Adham IM, Burkhardt E, Benahmed M, and Engel W (1993) Cloning of a cDNA for a novel insulin-like peptide of the testicular Leydig cells. J Biol Chem 268: 26668-26672.

Ahmad N, Wang W, Nair R, and Kapila S (2012) Relaxin induces matrixmetalloproteinases-9 and -13 via RXFP1: induction of MMP-9 involves the PI3K ERK, Akt and PKC- $\zeta$ pathways. Mol Cell Endocrinol 363:46-61.

Ahokas RA, Sibai BM, and Anderson GD (1989) Lack of evidence of a vasodepressor role for relaxin in spontaneously hypertensive and normotensive pregnant rats. Am J Obstet Gynecol 161:618-622.

Ahrén B (2009) Islet G protein-coupled receptors as potential targets for treatment of type 2 diabetes. Nat Rev Drug Discov 8:369-385.

Alexander BT, Miller MT, Kassab S, Novak J, Reckelhoff JF, Kruckeberg WC, and Granger JP (1999) Differential expression of renal nitric oxide synthase isoforms during pregnancy in rats. Hypertension 33:435-439.

Alexiou K, Matschke K, Westphal A, Stangl K, and Dschietzig T (2010) Relaxin is a candidate drug for lung preservation: relaxin-induced protection of rat lungs from ischemia-reperfusion injury. J Heart Lung Transplant 29:454-460. 
Alexiou K, Wilbring M, Matschke K, and Dschietzig T (2013) Relaxin protects rat lungs from ischemia-reperfusion injury via inducible NO synthase: role of ERK-1/2, PI3K, and forkhead transcription factor FKHRL1. PLoS ONE 8:e75592.

Alleva DG, Kaser SB, Monroy MA, Fenton MJ, and Beller DI (1997) IL-15 functions as a potent autocrine regulator of macrophage proinflammatory cytokine production: evidence for differential receptor subunit utilization associated with stimulation or inhibition. J Immunol 159:2941-2951.

Alvarez-Jaimes L, Sutton SW, Nepomuceno D, Motley ST, Cik M, Stocking E, Shoblock J, and Bonaventure P (2012) In vitro pharmacological characterization of RXFP3 allosterism: an example of probe dependency. PLoS ONE 7:e30792.

Anand-Ivell R and Ivell R (2014) Regulation of the reproductive cycle and early pregnancy by relaxin family peptides. Mol Cell Endocrinol 382:472-479.

Anand-Ivell R, Tremellen K, Dai Y, Heng K, Yoshida M, Knight PG, Hale GE, and Ivell R (2013) Circulating insulin-like factor 3 (INSL3) in healthy and infertile women. Hum Reprod 28:3093-3102.

Anand-Ivell RJ, Relan V, Balvers M, Coiffec-Dorval I, Fritsch M, Bathgate RA, and Ivell R (2006) Expression of the insulin-like peptide 3 (INSL3) hormonereceptor (LGR8) system in the testis. Biol Reprod 74:945-953.

Anand-Ivell R, Heng K, Bartsch O, and Ivell R (2007) Relaxin signalling in THP-1 cells uses a novel phosphotyrosine-dependent pathway. Mol Cell Endocrinol 272:1-13.

Arborelius L, Owens MJ, Plotsky PM, and Nemeroff CB (1999) The role of corticotropinreleasing factor in depression and anxiety disorders. J Endocrinol 160:1-12.

Arora A and Scholar EM (2005) Role of tyrosine kinase inhibitors in cancer therapy. $J$ Pharmacol Exp Ther 315:971-979.

Ascoli M, Fanelli F, and Segaloff DL (2002) The lutropin/choriogonadotropin receptor, a 2002 perspective. Endocr Rev 23:141-174.

Baccari MC, Bani D, Bigazzi M, and Calamai F (2004) Influence of relaxin on the neurally induced relaxant responses of the mouse gastric fundus. Biol Reprod $\mathbf{7 1}$ $1325-1329$

Baccari MC, Nistri S, Vannucchi MG, Calamai F, and Bani D (2007) Reversal by relaxin of altered ileal spontaneous contractions in dystrophic ( $\mathrm{mdx}$ ) mice through a nitric oxide-mediated mechanism. Am J Physiol Regul Integr Comp Physiol $\mathbf{2 9 3}$ R662-R668.

Baillie GS, Scott JD, and Houslay MD (2005) Compartmentalisation of phosphodiesterases and protein kinase A: opposites attract. FEBS Lett 579:3264-3270.

Baker JG and Hill SJ (2007) Multiple GPCR conformations and signalling pathways: implications for antagonist affinity estimates. Trends Pharmacol Sci 28:374-381.

Banerjee A, Shen PJ, Ma S, Bathgate RA, and Gundlach AL (2010) Swim stress excitation of nucleus incertus and rapid induction of relaxin-3 expression via CRF1 activation. Neuropharmacology 58:145-155.

Bani D (1997) Relaxin and breast cancer. Bull Cancer 84:179-182.

Bani D, Bigazzi M, Masini E, Bani G, and Sacchi TB (1995a) Relaxin depresses platelet aggregation: in vitro studies on isolated human and rabbit platelets. Lab Invest 73:709-716.

Bani D, Failli P, Bello MG, Thiemermann C, Bani Sacchi T, Bigazzi M, and Masini E (1998a) Relaxin activates the L-arginine-nitric oxide pathway in vascular smooth muscle cells in culture. Hypertension 31:1240-1247.

Bani D, Masini E, Bello MG, Bigazzi M, and Sacchi TB (1998b) Relaxin protects against myocardial injury caused by ischemia and reperfusion in rat heart. Am J Pathol 152:1367-1376.

Bani D, Maurizi M, and Bigazzi M (1995b) Original article: relaxin reduces the number of circulating platelets and depresses platelet release from megakaryocytes: studies in rats. Platelets 6:330-335.

Bani D, Nistri S, Quattrone S, Bigazzi M, and Sacchi TB (2001) Relaxin causes changes of the liver. In vivo studies in rats. Horm Metab Res 33:175-180.

Bani G, Bani Sacchi T, Bigazzi M, and Bianchi S (1988) Effects of relaxin on the microvasculature of mouse mammary gland. Histol Histopathol 3:337-343.

Bani-Sacchi T, Bigazzi M, Bani D, Mannaioni PF, and Masini E (1995) Relaxininduced increased coronary flow through stimulation of nitric oxide production. Br J Pharmacol 116:1589-1594.

Bartsch O, Bartlick B, and Ivell R (2001) Relaxin signalling links tyrosine phosphorylation to phosphodiesterase and adenylyl cyclase activity. Mol Hum Reprod 7: 799-809

Bates P, Young JAT, and Varmus HE (1993) A receptor for subgroup A Rous sarcoma virus is related to the low density lipoprotein receptor. Cell 74:1043-1051.

Bathgate R, Balvers M, Hunt N, and Ivell R (1996) Relaxin-like factor gene is highly expressed in the bovine ovary of the cycle and pregnancy: sequence and messenger ribonucleic acid analysis. Biol Reprod 55:1452-1457.

Bathgate R, Moniac N, Bartlick B, Schumacher M, Fields M, and Ivell R (1999) Expression and regulation of relaxin-like factor gene transcripts in the bovine ovary: differentiation-dependent expression in theca cell cultures. Biol Reprod $\mathbf{6 1}$ : 1090-1098

Bathgate RA, Halls ML, van der Westhuizen ET, Callander GE, Kocan M, and Summers RJ (2013a) Relaxin family peptides and their receptors. Physiol Rev 93:405-480.

Bathgate RA, Ivell R, Sanborn BM, Sherwood OD, and Summers RJ (2006a) International Union of Pharmacology LVII: recommendations for the nomenclature of receptors for relaxin family peptides. Pharmacol Rev 58:7-31.

Bathgate RA, Lekgabe ED, McGuane JT, Su Y, Pham T, Ferraro T, Layfield S, Hannan RD, Thomas WG, Samuel CS, et al. (2008) Adenovirus-mediated delivery of relaxin reverses cardiac fibrosis. Mol Cell Endocrinol 280:30-38.

Bathgate RA, Lin F, Hanson NF, Otvos L Jr, Guidolin A, Giannakis C, Bastiras S Layfield SL, Ferraro T, Ma S, et al. (2006b) Relaxin-3: improved synthesis strategy and demonstration of its high-affinity interaction with the relaxin receptor LGR7 both in vitro and in vivo. Biochemistry 45:1043-1053.

Bathgate RA, Oh MH, Ling WJ, Kaas Q, Hossain MA, Gooley PR, and Rosengren KJ (2013b) Elucidation of relaxin-3 binding interactions in the extracellular loops of RXFP3. Front Endocrinol (Lausanne) 4:13.

Bathgate RA, Samuel CS, Burazin TC, Layfield S, Claasz AA, Reytomas IG, Dawson $\mathrm{NF}$, Zhao C, Bond C, Summers RJ, et al. (2002) Human relaxin gene 3 (H3) and the equivalent mouse relaxin (M3) gene. Novel members of the relaxin peptide family. $J$ Biol Chem 277:1148-1157.

Bathgate RAD, Hsueh AJW, and Sherwood OD (2006c) Physiology and molecular biology of the relaxin peptide family, in Knobil and Neill's Physiology of Reproduction (Neill JD ed), Academic Press, New York.

Belgi A, Bathgate RA, Kocan M, Patil N, Zhang S, Tregear GW, Wade JD, and Hossain MA (2013) Minimum active structure of insulin-like peptide 5. J Med Chem 56:9509-9516.

Belgi A, Hossain MA, Shabanpoor F, Chan L, Zhang S, Bathgate RA, Tregear GW, and Wade JD (2011) Structure and function relationship of murine insulin-like peptide 5 (INSL5): free C-terminus is essential for RXFP4 receptor binding and activation. Biochemistry 50:8352-8361.

Bell RJ, Permezel M, MacLennan A, Hughes C, Healy D, and Brennecke S (1993) A randomized, double-blind, placebo-controlled trial of the safety of vaginal recombinant human relaxin for cervical ripening. Obstet Gynecol 82:328-333.

Bennett RG (2009) Relaxin and its role in the development and treatment of fibrosis. Transl Res 154:1-6.

Bergers G, Brekken R, McMahon G, Vu TH, Itoh T, Tamaki K, Tanzawa K, Thorpe P, Itohara S, Werb Z, et al. (2000) Matrix metalloproteinase-9 triggers the angiogenic switch during carcinogenesis. Nat Cell Biol 2:737-744.

Bhushan S, Kondo K, Polhemus DJ, Otsuka H, Nicholson CK, Tao YX, Huang H, Georgiopoulou VV, Murohara T, Calvert JW, et al. (2014) Nitrite therapy improves left ventricular function during heart failure via restoration of nitric oxidemediated cytoprotective signaling. Circ Res 114:1281-1291.

Bialek J, Kunanuvat U, Hombach-Klonisch S, Spens A Stetefeld J, Sunley K, Lippert D, Wilkins JA, Hoang-Vu C, and Klonisch T (2011) Relaxin enhances the collagenolytic activity and in vitro invasiveness by upregulating matrix metalloproteinase in human thyroid carcinoma cells. Mol Cancer Res 9:673-687.

Bigazzi M, Brandi ML, Bani G, and Sacchi TB (1992) Relaxin influences the growth of MCF-7 breast cancer cells. Mitogenic and antimitogenic action depends on peptide concentration. Cancer 70:639-643.

Bigazzi M, Del Mese A, Petrucci F, Casali R, and Novelli GP (1986) The local administration of relaxin induces changes in the microcirculation of the rat mesocaecum. Acta Endocrinol (Copenh) 112:296-299.

Binder C, Hagemann T, Husen B, Schulz M, and Einspanier A (2002) Relaxin enhances in-vitro invasiveness of breast cancer cell lines by up-regulation of matrix metalloproteases. Mol Hum Reprod 8:789-796.

Binder C, Simon A, Binder L, Hagemann T, Schulz M, Emons G, Trümper L, and Einspanier A (2004) Elevated concentrations of serum relaxin are associated with metastatic disease in breast cancer patients. Breast Cancer Res Treat 87: 157-166.

Bitto A, Irrera N, Minutoli L, Calò M, Lo Cascio P, Caccia P, Pizzino G, Pallio G, Micali A, Vaccaro M, et al. (2013) Relaxin improves multiple markers of wound healing and ameliorates the disturbed healing pattern of genetically diabetic mice. Clin Sci (Lond) 125:575-585.

Blasiak A, Blasiak T, Lewandowski MH, Hossain MA, Wade JD, and Gundlach AL (2013) Relaxin-3 innervation of the intergeniculate leaflet of the rat thalamus - neuronal tracttracing and in vitro electrophysiological studies. Eur J Neurosci 37:1284-1294.

Boehnert MU, Armbruster FP, and Hilbig H (2008) Relaxin as a protective substance in preservation solutions for organ transplantation, as shown in an isolated perfused rat liver model. Transplant Proc 40:978-980.

Boehnert MU, Armbruster FP and Hilbig H (2009) Relaxin as a protective substance in the preserving solution for liver transplantation: spectrophotometric in vivo imaging of local oxygen supply in an isolated perfused rat liver model. Ann NY Acad Sci 1160:320-321.

Boehnert MU, Hilbig H, and Armbruster FP (2005) Relaxin as an additional protective substance in preserving and reperfusion solution for liver transplantation, shown in a model of isolated perfused rat liver. Ann N Y Acad Sci 1041:434-440. Boels K and Schaller HC (2003) Identification and characterisation of GPR100 as a novel human G-protein-coupled bradykinin receptor. Br J Pharmacol 140:932-938.

Bogatcheva NV, Ferlin A, Feng S, Truong A, Gianesello L, Foresta C, and Agoulnik AI (2007) T222P mutation of the insulin-like 3 hormone receptor LGR8 is associated with testicular maldescent and hinders receptor expression on the cell surface membrane. Am J Physiol Endocrinol Metab 292:E138-E144.

Bogzil AH, Eardley R, and Ashton N (2005) Relaxin-induced changes in renal sodium excretion in the anesthetized male rat. Am J Physiol Regul Integr Comp Physiol 288:R322-R328.

Böhm M, Eschenhagen T, Gierschik P, Larisch K, Lensche H, Mende U, Schmitz W, Schnabel P, Scholz H, Steinfath M, et al. (1994) Radioimmunochemical quantification of $\mathrm{Gi} \alpha$ in right and left ventricles from patients with ischaemic and dilated cardiomyopathy and predominant left ventricular failure. $J$ Mol Cell Cardiol 26:133-149.

Bonacchi M, Nistri S, Nanni C, Gelsomino S, Pini A, Cinci L, Maiani M, ZecchiOrlandini S, Lorusso R, Fanti S, et al. (2009) Functional and histopathologica improvement of the post-infarcted rat heart upon myoblast cell grafting and relaxin therapy. J Cell Mol Med 13 (9B):3437-3448.

Bond CP, Parry LJ, Samuel CS, Gehring HM, Lederman FL, Rogers PA, and Summers RJ (2004) Increased expression of the relaxin receptor (LGR7) in human endometrium during the secretory phase of the menstrual cycle. $J$ Clin Endocrinol Metab 89:3477-3485.

Bonner JS, Lantier L, Hocking KM, Kang L, Owolabi M, James FD, Bracy DP Brophy CM, and Wasserman DH (2013) Relaxin treatment reverses insulin resistance in mice fed a high-fat diet. Diabetes 62:3251-3260.

Braddon SA (1978) Relaxin-dependent adenosine $6^{\prime}, 5^{\prime}$-monophosphate concentration changes in the mouse pubic symphysis. Endocrinology 102:1292-1299.

Brecht A, Bartsch C, Baumann G, Stangl K, and Dschietzig T (2011) Relaxin inhibits early steps in vascular inflammation. Regul Pept 166:76-82.

Bruell S, Kong RC, Petrie EJ, Hoare B, Wade JD, Scott DJ, Gooley PR, and Bathgate RA (2013) Chimeric RXFP1 and RXFP2 receptors highlight the similar mechanism of activation utilizing their N-terminal low-density lipoprotein class A modules. Front Endocrinol (Lausanne) 4:171. 
Büllesbach EE and Schwabe C (1994) Functional importance of the A chain loop in relaxin and insulin. $J$ Biol Chem 269:13124-13128.

Büllesbach EE and Schwabe C (2002) The primary structure and the disulfide links of the bovine relaxin-like factor (RLF). Biochemistry 41:274-281.

Büllesbach EE and Schwabe C (2004) Synthetic cross-links arrest the C-terminal region of the relaxin-like factor in an active conformation. Biochemistry 43:8021-8028.

Büllesbach EE and Schwabe C (2005a) LGR8 signal activation by the relaxin-like factor. J Biol Chem 280:14586-14590.

Bullesbach EE and Schwabe C (2005b) The trap-like relaxin-binding site of the leucine-rich G-protein-coupled receptor 7. J Biol Chem 280:14051-14056.

Büllesbach EE, Yang S, and Schwabe C (1992) The receptor-binding site of human relaxin II. A dual prong-binding mechanism. J Biol Chem 267:22957-22960.

Burazin TC, Bathgate RA, Macris M, Layfield S, Gundlach AL, and Tregear GW (2002) Restricted, but abundant, expression of the novel rat gene-3 (R3) relaxin in the dorsal tegmental region of brain. $J$ Neurochem 82:1553-1557.

Burazin TC, Johnson KJ, Ma S, Bathgate RA, Tregear GW, and Gundlach AL (2005) Localization of LGR7 (relaxin receptor) mRNA and protein in rat forebrain: correlation with relaxin binding site distribution. Ann N Y Acad Sci 1041:205-210.

Burger LL and Sherwood OD (1995) Evidence that cellular proliferation contributes to relaxin-induced growth of both the vagina and the cervix in the pregnant rat. Endocrinology 136:4820-4826.

Burger LL and Sherwood OD (1998) Relaxin increases the accumulation of new epithelial and stromal cells in the rat cervix during the second half of pregnancy. Endocrinology 139:3984-3995.

Burnicka-Turek O, Mohamed BA, Shirneshan K, Thanasupawat T, Hombach-Klonisch $\mathrm{S}$, Klonisch T, and Adham IM (2012) INSL5-deficient mice display an alteration in glucose homeostasis and an impaired fertility. Endocrinology 153:4655-4665.

Callander GE and Bathgate RA (2010) Relaxin family peptide systems and the central nervous system. Cell Mol Life Sci 67:2327-2341.

Callander GE, Thomas WG, and Bathgate RA (2009) Prolonged RXFP1 and RXFP2 signaling can be explained by poor internalization and a lack of beta-arrestin recruitment. Am J Physiol Cell Physiol 296:C1058-C1066.

Campitiello MR, De Franciscis P, Mele D, Izzo G, Sinisi A, Delrio G, and Colacurci N (2011) Endometrial LGR7 expression during menstrual cycle. Fertil Steril 95: 2511-2514

Carey RM (2005) Cardiovascular and renal regulation by the angiotensin type 2 receptor: the AT2 receptor comes of age. Hypertension 45:840-844.

Carrell DT, Peterson CM, and Urry RL (1995) The binding of recombinant human relaxin to human spermatozoa. Endocr Res 21:697-707.

Carvalho LN, Cristovam PC, Passos CS, and Boim MA (2012) Mesangial cells cultured from pregnant rats display reduced reactivity to angiotensin II: the role of relaxin, nitric oxide and AT2 receptor. Cell Physiol Biochem 30:1456-1464.

Casten GG and Boucek RJ (1958) Use of relaxin in the treatment of scleroderma. $J$ Am Med Assoc 166:319-324.

Chabre M, Deterre P, and Antonny B (2009) The apparent cooperativity of some GPCRs does not necessarily imply dimerization. Trends Pharmacol Sci 30:182-187.

Chan LJ, Rosengren KJ, Layfield SL, Bathgate RA, Separovic F, Samuel CS, Hossain MA, and Wade JD (2012) Identification of key residues essential for the structural fold and receptor selectivity within the A-chain of human gene-2 (H2) relaxin. J Biol Chem 287:41152-41164.

Chan SL and Cipolla MJ (2011) Relaxin causes selective outward remodeling of brain parenchymal arterioles via activation of peroxisome proliferator-activated receptor- $\gamma$. FASEB J 25:3229-3239.

Cheah SH and Sherwood OD (1980) Target tissues for relaxin in the rat: tissue distribution of injected 125I-labeled relaxin and tissue changes in adenosine $3^{\prime}, 5^{\prime}$ monophosphate levels after in vitro relaxin incubation. Endocrinology 106:1203-1209.

Chen CZ, Southall N, Xiao J, Marugan JJ, Ferrer M, Hu X, Jones RE, Feng S, Agoulnik IU, Zheng W, et al. (2013) Identification of small-molecule agonists of human relaxin family receptor 1 (RXFP1) by using a homogenous cell-based cAMP assay. J Biomol Screen 18:670-677.

Chen GA, Huang JR, and Tseng L (1988) The effect of relaxin on cyclic adenosine 3',5'-monophosphate concentrations in human endometrial glandular epithelial cells. Biol Reprod 39:519-525.

Chen J, Kuei C, Sutton SW, Bonaventure P, Nepomuceno D, Eriste E, Sillard R, Lovenberg TW, and Liu C (2005) Pharmacological characterization of relaxin-3/ INSL7 receptors GPCR135 and GPCR142 from different mammalian species. $J$ Pharmacol Exp Ther 312:83-95.

Chen J, Qiu Q, Lohstroh PN, Overstreet JW, and Lasley BL (2003) Hormonal characteristics in the early luteal phase of conceptive and nonconceptive menstrual cycles. J Soc Gynecol Investig 10:27-31.

Cheung KL and Lafayette RA (2013) Renal physiology of pregnancy. Adv Chronic Kidney Dis 20:209-214.

Chillakuri CR, Sheppard D, Lea SM, and Handford PA (2012) Notch receptor-ligand binding and activation: insights from molecular studies. Semin Cell Dev Biol 23:421-428.

Chow BS, Chew EG, Zhao C, Bathgate RA, Hewitson TD, and Samuel CS (2012) Relaxin signals through a RXFP1-pERK-nNOS-NO-cGMP-dependent pathway to up-regulate matrix metalloproteinases: the additional involvement of iNOS. PLoS ONE 7:e42714.

Chow BS, Kocan M, Bosnyak S, Sarwar M, Wigg B, Jones ES, Widdop RE, Summers RJ, Bathgate RA, Hewitson TD, et al. (2014) Relaxin requires the angiotensin II type 2 receptor to abrogate renal interstitial fibrosis. Kidney Int 86:75-85.

Christopoulos A (2002) Allosteric binding sites on cell-surface receptors: novel targets for drug discovery. Nat Rev Drug Discov 1:198-210.

Christopoulos A, Changeux JP, Catterall WA, Fabbro D, Burris TP, Cidlowski JA Olsen RW, Peters JA, Neubig RR, Pin JP, et al. (2014) International union of basic and clinical pharmacology. XC. multisite pharmacology: recommendations for the nomenclature of receptor allosterism and allosteric ligands. Pharmacol Rev 66:918-947.

Collino M, Rogazzo M, Pini A, Benetti E, Rosa AC, Chiazza F, Fantozzi R, Bani D, and Masini E (2013) Acute treatment with relaxin protects the kidney against ischaemia/reperfusion injury. J Cell Mol Med 17:1494-1505.
Conklin D, Lofton-Day CE, Haldeman BA, Ching A, Whitmore TE, Lok S, and Jaspers S (1999) Identification of INSL5, a new member of the insulin superfamily. Genomics 60:50-56.

Conrad KP (2004) Mechanisms of renal vasodilation and hyperfiltration during pregnancy. J Soc Gynecol Investig 11:438-448.

Conrad KP (2010) Unveiling the vasodilatory actions and mechanisms of relaxin. Hypertension 56:2-9.

Conrad KP (2011) Maternal vasodilation in pregnancy: the emerging role of relaxin. Am J Physiol Regul Integr Comp Physiol 301:R267-R275.

Conrad KP, Debrah DO, Novak J, Danielson LA, and Shroff SG (2004) Relaxin modifies systemic arterial resistance and compliance in conscious, nonpregnant rats. Endocrinology 145:3289-3296.

Conrad KP and Novak J (2004) Emerging role of relaxin in renal and cardiovascular function. Am J Physiol Regul Integr Comp Physiol 287:R250-R261.

Conrad KP and Shroff SG (2011) Effects of relaxin on arterial dilation, remodeling, and mechanical properties. Curr Hypertens Rep 13:409-420.

Cools R, Roberts AC, and Robbins TW (2008) Serotoninergic regulation of emotional and behavioural control processes. Trends Cogn Sci 12:31-40.

Cosen-Binker LI, Binker MG, Cosen R, Negri G, and Tiscornia O (2006) Relaxin prevents the development of severe acute pancreatitis. World J Gastroenterol 12: $1558-1568$.

Cottrell GS, Padilla BE, Amadesi S, Poole DP, Murphy JE, Hardt M, Roosterman D, Steinhoff M, and Bunnett NW (2009) Endosomal endothelin-converting enzyme-1 a regulator of $\beta$-arrestin-dependent ERK signaling. J Biol Chem 284:22411-22425.

Coulson CC, Thorp JM Jr, Mayer DC, and Cefalo RC (1996) Central hemodynamic effects of recombinant human relaxin in the isolated, perfused rat heart model. Obstet Gynecol 87:610-612.

Cronin MJ, Malaska T, and Bakhit C (1987) Human relaxin increases cyclic AMP levels in cultured anterior pituitary cells. Biochem Biophys Res Commun 148:1246-1251.

Daly NL, Scanlon MJ, Djordjevic JT, Kroon PA, and Smith R (1995) Threedimensional structure of a cysteine-rich repeat from the low-density lipoprotein receptor. Proc Natl Acad Sci USA 92:6334-6338.

Danielson LA and Conrad KP (1995) Acute blockade of nitric oxide synthase inhibits renal vasodilation and hyperfiltration during pregnancy in chronically instrumented conscious rats. $J$ Clin Invest 96:482-490.

Danielson LA and Conrad KP (2003) Time course and dose response of relaxinmediated renal vasodilation, hyperfiltration, and changes in plasma osmolality in conscious rats. J Appl Physiol (1985) 95:1509-1514.

Danielson LA, Kercher LJ, and Conrad KP (2000) Impact of gender and endothelin on renal vasodilation and hyperfiltration induced by relaxin in conscious rats. Am J Physiol Regul Integr Comp Physiol 279:R1298-R1304.

Danielson LA, Sherwood OD, and Conrad KP (1999) Relaxin is a potent renal vasodilator in conscious rats. J Clin Invest 103:525-533.

Davis D, Liu X, and Segaloff DL (1995) Identification of the sites of N-linked glycosylation on the follicle-stimulating hormone (FSH) receptor and assessment of their role in FSH receptor function. Mol Endocrinol 9:159-170.

Davis RJ (2000) Signal transduction by the JNK group of MAP kinases. Cell 103: 239-252.

Debrah DO, Conrad KP, Danielson LA, and Shroff SG (2005) Effects of relaxin on systemic arterial hemodynamics and mechanical properties in conscious rats: sex dependency and dose response. J Appl Physiol (1985) 98:1013-1020.

Debrah DO, Debrah JE, Haney JL, McGuane JT, Sacks MS, Conrad KP, and Shroff SG (2011) Relaxin regulates vascular wall remodeling and passive mechanical properties in mice. J Appl Physiol (1985) 111:260-271.

Debrah DO, Novak J, Matthews JE, Ramirez RJ, Shroff SG, and Conrad KP (2006) Relaxin is essential for systemic vasodilation and increased global arterial compliance during early pregnancy in conscious rats. Endocrinology 147:5126-5131.

DeFea KA (2011) Beta-arrestins as regulators of signal termination and transduction: how do they determine what to scaffold? Cell Signal 23:621-629.

Defer N, Best-Belpomme M, and Hanoune J (2000) Tissue specificity and physiological relevance of various isoforms of adenylyl cyclase. Am $J$ Physiol Renal Physiol 279:F400-F416.

Del Borgo MP, Hughes RA, Bathgate RA, Lin F, Kawamura K, and Wade JD (2006) Analogs of insulin-like peptide 3 (INSL3) B-chain are LGR8 antagonists in vitro and in vivo. $J$ Biol Chem 281:13068-13074.

Dessauer CW (2009) Adenylyl cyclase-A-kinase anchoring protein complexes: the next dimension in cAMP signaling. Mol Pharmacol 76:935-941.

DiScipio RG, Gehring MR, Podack ER, Kan CC, Hugli TE, and Fey GH (1984) Nucleotide sequence of cDNA and derived amino acid sequence of human complement component C9. Proc Natl Acad Sci USA 81:7298-7302.

Dschietzig T, Alexiou K, Kinkel HT, Baumann G, Matschke K, and Stangl K (2011) The positive inotropic effect of relaxin-2 in human atrial myocardium is preserved in end-stage heart failure: role of G(i)-phosphoinositide-3 kinase signaling. J Card Fail 17:158-166.

Dschietzig T, Bartsch C, Baumann G, and Stangl K (2009a) RXFP1-inactive relaxin activates human glucocorticoid receptor: further investigations into the relaxin-GR pathway. Regul Pept 154:77-84.

Dschietzig T, Bartsch C, Kinkel T, Baumann G, and Stangl K (2005) Myocardial relaxin counteracts hypertrophy in hypertensive rats. Ann N Y Acad Sci 1041:441-443.

Dschietzig T, Bartsch C, Richter C, Laule M, Baumann G, and Stangl K (2003) Relaxin, a pregnancy hormone, is a functional endothelin-1 antagonist: attenuation of endothelin-1-mediated vasoconstriction by stimulation of endothelin type-B receptor expression via ERK-1/2 and nuclear factor-kappaB. Circ Res 92:32-40.

Dschietzig T, Bartsch C, Stangl V, Baumann G, and Stangl K (2004) Identification of the pregnancy hormone relaxin as glucocorticoid receptor agonist. FASEB $J$ 18: $1536-1538$

Dschietzig T, Bartsch C, Wessler S, Baumann G, and Stangl K (2009b) Autoregulation of human relaxin-2 gene expression critically involves relaxin and glucocorticoid receptor binding to glucocorticoid response half-sites in the relaxin-2 promoter. Regul Pept 155:163-173. 
Dschietzig T, Brecht A, Bartsch C, Baumann G, Stangl K, and Alexiou K (2012) Relaxin improves TNF- $\alpha$-induced endothelial dysfunction: the role of glucocorticoid receptor and phosphatidylinositol 3-kinase signalling. Cardiovasc Res 95:97-107.

Dschietzig T, Richter C, Bartsch C, Böhme C, Heinze D, Ott F, Zartnack F, Baumann G, and Stangl K (2001a) Flow-induced pressure differentially regulates endothelin-1, urotensin II, adrenomedullin, and relaxin in pulmonary vascular endothelium. Biochem Biophys Res Commun 289:245-251.

Dschietzig T, Richter C, Bartsch C, Laule M, Armbruster FP, Baumann G, and Stangl K (2001b) The pregnancy hormone relaxin is a player in human heart failure. FASEB J 15:2187-2195.

Dschietzig T, Teichman S, Unemori E, Wood S, Boehmer J, Richter C, Baumann G, and Stangl K (2009c) Intravenous recombinant human relaxin in compensated heart failure: a safety, tolerability, and pharmacodynamic trial. J Card Fail 15: 182-190.

Du XJ, Bathgate RA, Samuel CS, Dart AM, and Summers RJ (2010) Cardiovascular effects of relaxin: from basic science to clinical therapy. Nat Rev Cardiol 7:48-58.

Eddie LW, Cameron IT, Leeton JF, Healy DL, and Renou P (1990a) Ovarian relaxin is not essential for dilatation of cervix. Lancet 336:243.

Eddie LW, Martinez F, Healy DL, Sutton B, Bell RJ, and Tregear GW (1990b) Relaxin in sera during the luteal phase of in-vitro fertilization cycles. $\mathrm{Br} J$ Obstet Gynaecol 97:215-220.

Efendiev R, Samelson BK, Nguyen BT, Phatarpekar PV, Baameur F, Scott JD, and Dessauer CW (2010) AKAP79 interacts with multiple adenylyl cyclase (AC) isoforms and scaffolds AC5 and -6 to alpha-amino-3-hydroxyl-5-methyl-4-isoxazolepropionate (AMPA) receptors. J Biol Chem 285:14450-14458.

Egan CT, Herrick-Davis K, and Teitler M (1998) Creation of a constitutively activated state of the 5 -hydroxytryptamine $2 \mathrm{~A}$ receptor by site-directed mutagenesis: inverse agonist activity of antipsychotic drugs. J Pharmacol Exp Ther 286:85-90.

Eigenbrot C, Randal M, Quan C, Burnier J, O'Connell L, Rinderknecht E, and Kossiakoff AA (1991) X-ray structure of human relaxin at $1.5 \mathrm{~A}$. Comparison to insulin and implications for receptor binding determinants. J Mol Biol 221:15-21.

Erikson MS and Unemori EN (2001) Relaxin clinical trials in systemic sclerosis, in Relaxin 2000: Proceedings of the 3rd International Conference on Relaxin and Related Peptides (Tregear GW, Ivell R, Bathgate RA, and Wade JD eds) pp 373-382, Kluwer, Amsterdam.

Eschenhagen T, Mende U, Nose M, Schmitz W, Scholz H, Haverich A, Hirt S, Döring V, Kalmár P, Höppner W, et al. (1992) Increased messenger RNA level of the inhibitory $\mathrm{G}$ protein $\alpha$ subunit $\mathrm{Gi} \alpha-_{2}$ in human end-stage heart failure. Circ Res 70:688-696.

Evans BA, Sato M, Sarwar M, Hutchinson DS, and Summers RJ (2010) Liganddirected signalling at $\beta$-adrenoceptors. Br J Pharmacol 159:1022-1038.

Evans MI, Dougan MB, Moawad AH, Evans WJ, Bryant-Greenwood GD, and Greenwood FC (1983) Ripening of the human cervix with porcine ovarian relaxin. Am J Obstet Gynecol 147:410-414.

Failli P, Nistri S, Quattrone S, Mazzetti L, Bigazzi M, Sacchi TB, and Bani D (2002) Relaxin up-regulates inducible nitric oxide synthase expression and nitric oxide generation in rat coronary endothelial cells. FASEB J 16:252-254.

Familari M, Vu D, and Parry LJ (2009) Regulation of Rxfp2 (Lgr8) expression in the mouse fetal kidney by the transcription factor Pod1 (Tcf 21). Ann N Y Acad Sci 1160:317-319.

Fan YH, Dong H, Pan Q, Cao YJ, Li H, and Wang HC (2011) Notch signaling may negatively regulate neonatal rat cardiac fibroblast-myofibroblast transformation. Physiol Res 60:739-748.

Fei DT, Gross MC, Lofgren JL, Mora-Worms M, and Chen AB (1990) Cyclic AMP response to recombinant human relaxin by cultured human endometrial cellsa specific and high throughput in vitro bioassay. Biochem Biophys Res Commun 170:214-222.

Feng S, Agoulnik IU, Bogatcheva NV, Kamat AA, Kwabi-Addo B, Li R, Ayala G, Ittmann MM, and Agoulnik AI (2007) Relaxin promotes prostate cancer progression. Clin Cancer Res 13:1695-1702.

Feng S, Agoulnik IU, Truong A, Li Z, Creighton CJ, Kaftanovskaya EM, Pereira R, Han HD, Lopez-Berestein G, Klonisch T, et al. (2010) Suppression of relaxin receptor RXFP1 decreases prostate cancer growth and metastasis. Endocr Relat Cancer 17:1021-1033.

Feng S, Bogatcheva NV, Kamat AA, Truong A, and Agoulnik AI (2006) Endocrine effects of relaxin overexpression in mice. Endocrinology 147:407-414.

Ferlin A, Menegazzo M, Gianesello L, Selice R, and Foresta C (2012) Effect of relaxin on human sperm functions. J Androl 33:474-482.

Ferlin A, Pepe A, Gianesello L, Garolla A, Feng S, Facciolli A, Morello R, Agoulnik AI, and Foresta C (2009) New roles for INSL3 in adults. Ann N Y Acad Sci 1160:215-218.

Ferlin A, Pepe A, Gianesello L, Garolla A, Feng S, Giannini S, Zaccolo M, Facciolli A Morello R, Agoulnik AI, et al. (2008) Mutations in the insulin-like factor 3 receptor are associated with osteoporosis. J Bone Miner Res 23:683-693.

Ferlin A, Selice R, Carraro U, and Foresta C (2013) Testicular function and bone metabolism-beyond testosterone. Nat Rev Endocrinol 9:548-554.

Fernandez-Patron C, Radomski MW, and Davidge ST (1999) Vascular matrix metalloproteinase-2 cleaves big endothelin-1 yielding a novel vasoconstrictor. Circ Res 85:906-911.

Fernandez-Patron C, Stewart KG, Zhang Y, Koivunen E, Radomski MW, and Davidge ST (2000) Vascular matrix metalloproteinase-2-dependent cleavage of calcitonin gene-related peptide promotes vasoconstriction. Circ Res 87:670-676.

Ferreira VM, Gomes TS, Reis LA, Ferreira AT, Razvickas CV, Schor N, and Boim MA (2009) Receptor-induced dilatation in the systemic and intrarenal adaptation to pregnancy in rats. PLoS ONE 4:e4845.

Filonzi M, Cardoso LC, Pimenta MT, Queiróz DB, Avellar MC, Porto CS, and Lazari MF (2007) Relaxin family peptide receptors Rxfp1 and Rxfp2: mapping of the mRNA and protein distribution in the reproductive tract of the male rat. Reprod Biol Endocrinol 5:29.

Fisher C, Berry C, Blue L, Morton JJ, and McMurray J (2003) N-terminal pro B type natriuretic peptide, but not the new putative cardiac hormone relaxin, predicts prognosis in patients with chronic heart failure. Heart 89:879-881.
Fisher C, MacLean M, Morecroft I, Seed A, Johnston F, Hillier C, and McMurray J (2002) Is the pregnancy hormone relaxin also a vasodilator peptide secreted by the heart? Circulation 106:292-295.

Foresta C, Bettella A, Vinanzi C, Dabrilli P, Meriggiola MC, Garolla A, and Ferlin A (2004) A novel circulating hormone of testis origin in humans. J Clin Endocrinol Metab 89:5952-5958.

Formigli L, Francini F, Nistri S, Margheri M, Luciani G, Naro F, Silvertown JD, Orlandini SZ, Meacci E, and Bani D (2009) Skeletal myoblasts overexpressing relaxin improve differentiation and communication of primary murine cardiomyocyte cell cultures. J Mol Cell Cardiol 47:335-345.

Fu P, Shen PJ, Zhao CX, Scott DJ, Samuel CS, Wade JD, Tregear GW, Bathgate RA, and Gundlach AL (2006) Leucine-rich repeat-containing G-protein-coupled receptor 8 in mature glomeruli of developing and adult rat kidney and inhibition by insulin-like peptide-3 of glomerular cell proliferation. J Endocrinol 189:397-408.

Funato N, Ohyama K, Kuroda T, and Nakamura M (2003) Basic helix-loop-helix transcription factor epicardin/capsulin/Pod-1 suppresses differentiation by negative regulation of transcription. J Biol Chem 278:7486-7493.

Gåfvels ME, Caird M, Britt D, Jackson CL, Patterson D, and Strauss JFI 3rd (1993) Cloning of a cDNA encoding a putative human very low density lipoprotein/ apolipoprotein E receptor and assignment of the gene to chromosome 9pter-p23. Somat Cell Mol Genet 19:557-569.

Gambineri A, Patton L, De Iasio R, Palladoro F, Pagotto U, and Pasquali R (2007) Insulin-like factor 3: a new circulating hormone related to luteinizing hormonedependent ovarian hyperandrogenism in the polycystic ovary syndrome. $J \mathrm{Clin}$ Endocrinol Metab 92:2066-2073.

Gandley RE, Conrad KP, and McLaughlin MK (2001) Endothelin and nitric oxide mediate reduced myogenic reactivity of small renal arteries from pregnant rats. Am J Physiol Regul Integr Comp Physiol 280:R1-R7.

Ganella DE, Callander GE, Ma S, Bye CR, Gundlach AL, and Bathgate RA (2013a) Modulation of feeding by chronic rAAV expression of a relaxin-3 peptide agonist in rat hypothalamus. Gene Ther 20:703-716.

Ganella DE, Ma S, and Gundlach AL (2013b) Relaxin-3/RXFP3 signaling and neuroendocrine function - a perspective on extrinsic hypothalamic control. Front Endocrinol (Lausanne) 4:128.

Ganesan A, Klonisch T, McGuane JT, Feng S, Agoulnik AI, and Parry LJ (2009) Normal prostate morphology in relaxin-mutant mice. Reprod Fertil Dev 21 440-450.

Garber SL, Mirochnik Y, Brecklin CS, Unemori EN, Singh AK, Slobodskoy L, Grove BH, Arruda JA, and Dunea G (2001) Relaxin decreases renal interstitial fibrosis and slows progression of renal disease. Kidney Int 59:876-882.

Garrick NA, Hill JL, Szele FG, Tomai TP, Gold PW, and Murphy DL (1987) Corticotropin-releasing factor: a marked circadian rhythm in primate cerebrospinal fluid peaks in the evening and is inversely related to the cortisol circadian rhythm. Endocrinology 121:1329-1334.

Gelman S (2008) Venous function and central venous pressure: a physiologic story. Anesthesiology 108:735-748.

Ghattas MH, Mehanna ET, Mesbah NM, and Abo-Elmatty DM (2013) Relaxin-3 is associated with metabolic syndrome and its component traits in women. Clin Biochem 46:45-48.

Glister C, Satchell L, Bathgate RA, Wade JD, Dai Y, Ivell R, Anand-Ivell R, Rodgers RJ, and Knight PG (2013) Functional link between bone morphogenetic proteins and insulin-like peptide 3 signaling in modulating ovarian androgen production. Proc Natl Acad Sci USA 110:E1426-E1435.

Glogowska A, Kunanuvat U, Stetefeld J, Patel TR, Thanasupawat T, Krcek J, Weber E, Wong GW, Del Bigio MR, Hoang-Vu C, et al. (2013) C1q-tumour necrosis factorrelated protein 8 (CTRP8) is a novel interaction partner of relaxin receptor RXFP1 in human brain cancer cells. $J$ Pathol 231:466-479.

Gooi JH, Richardson ML, Jelinic M, Girling JE, Wlodek ME, Tare M, and Parry LJ (2013) Enhanced uterine artery stiffness in aged pregnant relaxin mutant mice is reversed with exogenous relaxin treatment. Biol Reprod 89:18.

Gorlov IP, Kamat A, Bogatcheva NV, Jones E, Lamb DJ, Truong A, Bishop CE, McElreavey K, and Agoulnik AI (2002) Mutations of the GREAT gene cause cryptorchidism. Hum Mol Genet 11:2309-2318.

Goto M, Swanson LW, and Canteras NS (2001) Connections of the nucleus incertus. $J$ Comp Neurol 438:86-122.

Grosse J, Heffron H, Burling K, Akhter Hossain M, Habib AM, Rogers GJ, Richards P, Larder R, Rimmington D, Adriaenssens AA, et al. (2014) Insulin-like peptide 5 is an orexigenic gastrointestinal hormone. Proc Natl Acad Sci USA 111:11133-11138.

Hall K (1960) Modification by relaxin of the response of the reproductive tract of mice to oestradiol and progesterone. J Endocrinol 20:355-364.

Halls ML (2012) Constitutive formation of an RXFP1-signalosome: a novel paradigm in GPCR function and regulation. Br J Pharmacol 165:1644-1658.

Halls ML, Bathgate RA, Sudo S, Kumagai J, Bond CP, and Summers RJ (2005a) Identification of binding sites with differing affinity and potency for relaxin analogues on LGR7 and LGR8 receptors. Ann N Y Acad Sci 1041:17-21.

Halls ML, Bathgate RA, and Summers RJ (2006) Relaxin family peptide receptors RXFP1 and RXFP2 modulate cAMP signaling by distinct mechanisms. Mol Phar macol 70:214-226.

Halls ML, Bathgate RA, and Summers RJ (2007a) Comparison of signaling pathways activated by the relaxin family peptide receptors, RXFP1 and RXFP2, using reporter genes. J Pharmacol Exp Ther 320:281-290.

Halls ML, Bond CP, Sudo S, Kumagai J, Ferraro T, Layfield S, Bathgate RA, and Summers RJ (2005b) Multiple binding sites revealed by interaction of relaxin family peptides with native and chimeric relaxin family peptide receptors 1 and 2 (LGR7 and LGR8). J Pharmacol Exp Ther 313:677-687.

Halls ML and Cooper DM (2010) Sub-picomolar relaxin signalling by a pre-assembled RXFP1, AKAP79, AC2, $\beta$-arrestin 2, PDE4D3 complex. EMBO J 29:2772-2787.

Halls ML, Hewitson TD, Moore XL, Du XJ, Bathgate RA, and Summers RJ (2009a) Relaxin activates multiple cAMP signaling pathway profiles in different target cells. Ann N Y Acad Sci 1160:108-111. 
Halls ML, van der Westhuizen ET, Bathgate RA, and Summers RJ (2007b) Relaxin family peptide receptors-former orphans reunite with their parent ligands to activate multiple signalling pathways. $\mathrm{Br} J$ Pharmacol 150:677-691.

Halls ML, van der Westhuizen ET, Wade JD, Evans BA, Bathgate RA, and Summers RJ (2009b) Relaxin family peptide receptor (RXFP1) coupling to $G(\alpha)_{\text {i3 }}$ involves the C-terminal Arg752 and localization within membrane Raft Microdomains. Mol Pharmacol 75:415-428.

Han X, Habuchi Y, and Giles WR (1994) Relaxin increases heart rate by modulating calcium current in cardiac pacemaker cells. Circ Res 74:537-541.

Hartley BJ, Scott DJ, Callander GE, Wilkinson TN, Ganella DE, Kong CK, Layfield S, Ferraro T, Petrie EJ, and Bathgate RA (2009) Resolving the unconventional mechanisms underlying RXFP1 and RXFP2 receptor function. Ann N Y Acad Sci 1160:67-73.

Haugaard-Jönsson LM, Hossain MA, Daly NL, Bathgate RA, Wade JD, Craik DJ, and Rosengren KJ (2008) Structure of the R3/I5 chimeric relaxin peptide, a selective GPCR135 and GPCR142 agonist. J Biol Chem 283:23811-23818.

Haugaard-Jönsson LM, Hossain MA, Daly NL, Craik DJ, Wade JD, and Rosengren KJ (2009) Structure of human insulin-like peptide 5 and characterization of conserved hydrogen bonds and electrostatic interactions within the relaxin framework. Biochem J 419:619-627.

Haugaard-Kedström LM, Shabanpoor F, Hossain MA, Clark RJ, Ryan PJ, Craik DJ, Gundlach AL, Wade JD, Bathgate RA, and Rosengren KJ (2011) Design, synthesis, and characterization of a single-chain peptide antagonist for the relaxin-3 receptor RXFP3. J Am Chem Soc 133:4965-4974.

Heeg MHJ, Koziolek MJ, Vasko R, Schaefer L, Sharma K, Müller GA, and Strutz F (2005) The antifibrotic effects of relaxin in human renal fibroblasts are mediated in part by inhibition of the Smad2 pathway. Kidney Int 68:96-109.

Heng K, Ivell R, Wagaarachchi P, and Anand-Ivell R (2008) Relaxin signalling in primary cultures of human myometrial cells. Mol Hum Reprod 14:603-611.

Heringlake M, Kox T, Poeling J, Klaus S, Hanke T, Franz N, Eberhardt F, Heinze H, Armbruster FP, and Bahlmann L (2009) The effects of physical exercise on plasma levels of relaxin, NTproANP, and NTproBNP in patients with ischemic heart disease. Eur J Med Res 14:106-112.

Herrick-Davis K, Egan C, and Teitler M (1997) Activating mutations of the serotonin $5-\mathrm{HT}_{2 \mathrm{C}}$ receptor. $J$ Neurochem 69:1138-1144.

Herz J, Hamann U, Rogne S, Myklebost O, Gausepohl H, and Stanley KK (1988) Surface location and high affinity for calcium of a 500-kd liver membrane protein closely related to the LDL-receptor suggest a physiological role as lipoprotein receptor. EMBO J 7:4119-4127.

Hida T, Takahashi E, Shikata K, Hirohashi T, Sawai T, Seiki T, Tanaka H, Kawai T, Ito O, Arai T, et al. (2006) Chronic intracerebroventricular administration of relaxin-3 increases body weight in rats. J Recept Signal Transduct Res 26:147-158.

Hisaw FL (1926) Experimental relaxation of the pubic ligament of the guinea pig. Proc Soc Exp Biol Med 23:661-663.

Hjälm G, Murray E, Crumley G, Harazim W, Lundgren S, Onyango I, Ek B, Larsson M, Juhlin C, Hellman P, et al. (1996) Cloning and sequencing of human gp330, a $\mathrm{Ca}(2+)$-binding receptor with potential intracellular signaling properties. Eur $J$ Biochem 239:132-137.

Hombach-Klonisch S, Bialek J, Radestock Y, Truong A, Agoulnik AI, Fiebig B, Willing C, Weber E, Hoang-Vu C, and Klonisch T (2010) INSL3 has tumorpromoting activity in thyroid cancer. Int $J$ Cancer 127:521-531.

Hombach-Klonisch S, Bialek J, Trojanowicz B, Weber E, Holzhausen H-J, Silvertown JD, Summerlee AJ, Dralle H, Hoang-Vu C, and Klonisch T (2006) Relaxin enhances the oncogenic potential of human thyroid carcinoma cells. Am J Pathol 169:617-632.

Hombach-Klonisch S, Hoang-Vu C, Kehlen A, Hinze R, Holzhausen HJ, Weber E, Fischer B, Dralle H, and Klonisch T (2003) INSL-3 is expressed in human hyperplastic and neoplastic thyrocytes. Int J Oncol 22:993-1001.

Hopkins EJ, Layfield S, Ferraro T, Bathgate RA, and Gooley PR (2007) The NMR solution structure of the relaxin (RXFP1) receptor lipoprotein receptor class A module and identification of key residues in the N-terminal region of the module that mediate receptor activation. J Biol Chem 282:4172-4184.

Horton JS, Yamamoto SY, and Bryant-Greenwood GD (2011) Relaxin modulates proinflammatory cytokine secretion from human decidual macrophages. Biol Reprod 85:788-797.

Hosken IT, Sutton SW, Smith CM, and Gundlach AL (2014) Relaxin-3 receptor (Rxfp3) gene knockout mice display reduced running wheel activity: Implications for role of relaxin-3/RXFP3 signalling in sustained arousal. Behav Brain Res 278C 167-175 10.1016/j.bbr.2014.09.028

Hossain MA, Bathgate RA, Rosengren KJ, Shabanpoor F, Zhang S, Lin F, Tregear GW, and Wade JD (2009) The structural and functional role of the B-chain C-terminal arginine in the relaxin-3 peptide antagonist, R3(BDelta23-27)R/I5. Chem Biol Drug Des 73:46-52.

Hossain MA, Rosengren KJ, Haugaard-Jönsson LM, Zhang S, Layfield S, Ferraro T, Daly NL, Tregear GW, Wade JD, and Bathgate RA (2008) The A-chain of human relaxin family peptides has distinct roles in the binding and activation of the different relaxin family peptide receptors. J Biol Chem 283:17287-17297.

Hossain MA, Rosengren KJ, Samuel CS, Shabanpoor F, Chan LJ, Bathgate RA and Wade JD (2011) The minimal active structure of human relaxin-2. J Biol Chem 286:37555-37565.

Hossain MA, Samuel CS, Binder C, Hewitson TD, Tregear GW, Wade JD, and Bathgate RA (2010) The chemically synthesized human relaxin-2 analog, B-R13/17K H2, is an RXFP1 antagonist. Amino Acids 39:409-416.

Hossain MA and Wade JD (2010) The roles of the A- and B-chains of human relaxin-2 and -3 on their biological activity. Curr Protein Pept Sci 11:719-724.

Hsu CJ, McCormack SM, and Sanborn BM (1985) The effect of relaxin on cyclic adenosine $3^{\prime}, 5^{\prime}$-monophosphate concentrations in rat myometrial cells in culture. Endocrinology 116:2029-2035.

Hsu SY (2003) New insights into the evolution of the relaxin-LGR signaling system. Trends Endocrinol Metab 14:303-309.
Hsu SY, Kudo M, Chen T, Nakabayashi K, Bhalla A, van der Spek PJ, van Duin M, and Hsueh AJ (2000) The three subfamilies of leucine-rich repeat-containing G protein-coupled receptors (LGR): identification of LGR6 and LGR7 and the signaling mechanism for LGR7. Mol Endocrinol 14:1257-1271.

Hsu SY, Nakabayashi K, Nishi S, Kumagai J, Kudo M, Sherwood OD, and Hsueh AJ (2002) Activation of orphan receptors by the hormone relaxin. Science 295:671-674.

Huang Z, Rivas B, and Agoulnik AI (2012) Insulin-like 3 signaling is important for testicular descent but dispensable for spermatogenesis and germ cell survival in adult mice. Biol Reprod 87:143.

Ivell R and Anand-Ivell R (2009) Biology of insulin-like factor 3 in human reproduction. Hum Reprod Update 15:463-476.

Ivell R, Balvers M, Pohnke Y, Telgmann R, Bartsch O, Milde-Langosch K, Bamberger AM, and Einspanier A (2003) Immunoexpression of the relaxin receptor LGR7 in breast and uterine tissues of humans and primates. Reprod Biol Endocrinol 1:114

Ivell R and Bathgate RA (2002) Reproductive biology of the relaxin-like factor (RLF/INSL3). Biol Reprod 67:699-705.

Ivell R and Hartung S (2003) The molecular basis of cryptorchidism. Mol Hum Reprod 9:175-181.

Ivell R, Heng K, and Anand-Ivell R (2014) Insulin-like factor 3 and the HPG axis in the male. Front Endocrinol (Lausanne) 5:6.

Jarrett JC (2nd), Ballejo G, Saleem TH, Tsibris JC, and Spellacy WN (1984) The effect of prolactin and relaxin on insulin binding by adipocytes from pregnant women. Am J Obstet Gynecol 149:250-255.

Jelinic M, Leo CH, Post Uiterweer ED, Sandow SL, Gooi JH, Wlodek ME, Conrad KP, Parkington H, Tare M, and Parry LJ (2014) Localization of relaxin receptors in arteries and veins, and region-specific increases in compliance and bradykininmediated relaxation after in vivo serelaxin treatment. FASEB J 28:275-287.

Jeyabalan A, Kerchner LJ, Fisher MC, McGuane JT, Doty KD, and Conrad KP (2006 Matrix metalloproteinase-2 activity, protein, mRNA, and tissue inhibitors in small arteries from pregnant and relaxin-treated nonpregnant rats. J Appl Physiol (1985) 100:1955-1963.

Jeyabalan A, Novak J, Danielson LA, Kerchner LJ, Opett SL, and Conrad KP (2003) Essential role for vascular gelatinase activity in relaxin-induced renal vasodilation, hyperfiltration, and reduced myogenic reactivity of small arteries. Circ Res 93:1249-1257.

Jeyabalan A, Novak J, Doty KD, Matthews J, Fisher MC, Kerchner LJ, and Conrad KP (2007) Vascular matrix metalloproteinase-9 mediates the inhibition of myogenic reactivity in small arteries isolated from rats after short-term administration of relaxin. Endocrinology 148:189-197.

Johnson MR, Abdalla H, Allman AC, Wren ME, Kirkland A, and Lightman SL (1991) Relaxin levels in ovum donation pregnancies. Fertil Steril 56:59-61.

Johnson MR, Brooks AA, and Steer PJ (1996) The role of relaxin in the pregnancy associated reduction in plasma osmolality. Hum Reprod 11:1105-1108.

Johnston SE, Gratten J, Berenos C, Pilkington JG, Clutton-Brock TH, Pemberton JM, and Slate J (2013) Life history trade-offs at a single locus maintain sexually selected genetic variation. Nature 502:93-95.

Jones ES, Vinh A, McCarthy CA, Gaspari TA, and Widdop RE (2008) AT2 receptors: functional relevance in cardiovascular disease. Pharmacol Ther 120:292-316.

Kakouris H, Eddie LW, and Summers RJ (1992) Cardiac effects of relaxin in rats. Lancet 339:1076-1078.

Kamat AA, Feng S, Agoulnik IU, Kheradmand F, Bogatcheva NV, Coffey D, Sood AK and Agoulnik AI (2006) The role of relaxin in endometrial cancer. Cancer Biol Ther 5:71-77.

Kamat AA, Feng S, Bogatcheva NV, Truong A, Bishop CE, and Agoulnik AI (2004) Genetic targeting of relaxin and insulin-like factor 3 receptors in mice. Endocrinology 145:4712-4720.

Kawamura K, Kumagai J, Sudo S, Chun SY, Pisarska M, Morita H, Toppari J, Fu P, Wade JD, Bathgate RA et al, (2004) Paracrine regulation of mammalian oocyte maturation and male germ cell survival. Proc Natl Acad Sci USA 101:7323-7328.

Kenakin T and Miller LJ (2010) Seven transmembrane receptors as shapeshifting proteins: the impact of allosteric modulation and functional selectivity on new drug discovery. Pharmacol Rev 62:265-304.

Kerchner LJ, Novak J, Hanley-Yanez K, Doty KD, Danielson LA, and Conrad KP (2005) Evidence against the hypothesis that endothelial endothelin B receptor expression is regulated by relaxin and pregnancy. Endocrinology 146:2791-2797.

Kern A, Agoulnik AI, and Bryant-Greenwood GD (2007) The low-density lipoprotein class A module of the relaxin receptor (leucine-rich repeat containing G-protein coupled receptor 7): its role in signaling and trafficking to the cell membrane. Endocrinology 148:1181-1194.

Kern A and Bryant-Greenwood GD (2009) Characterization of relaxin receptor (RXFP1) desensitization and internalization in primary human decidual cells and RXFP1-transfected HEK293 cells. Endocrinology 150:2419-2428.

Kern A, Hubbard D, Amano A, and Bryant-Greenwood GD (2008) Cloning, expression, and functional characterization of relaxin receptor (leucine-rich repeat-containing g protein-coupled receptor 7) splice variants from human fetal membranes. Endocrinology 149:1277-1294.

Khanna D, Clements PJ, Furst DE, Korn JH, Ellman M, Rothfield N, Wigley FM, Moreland LW, Silver R, Kim YH, et al.; Relaxin Investigators and the Scleroderma Clinical Trials Consortium (2009) Recombinant human relaxin in the treatment of systemic sclerosis with diffuse cutaneous involvement: a randomized, double-blind, placebo-controlled trial. Arthritis Rheum 60:1102-1111.

Kjelsberg MA, Cotecchia S, Ostrowski J, Caron MG, and Lefkowitz RJ (1992) Constitutive activation of the $\alpha_{1 \mathrm{~B}}$-adrenergic receptor by all amino acid substitutions at a single site. Evidence for a region which constrains receptor activation. J Biol Chem 267:1430-1433.

Klonisch T, Müller-Huesmann H, Riedel M, Kehlen A, Bialek J, Radestock Y, Holzhausen HJ, Steger K, Ludwig M, Weidner W, et al. (2005) INSL3 in the benign hyperplastic and neoplastic human prostate gland. Int $J$ Oncol 27:307-315.

Kocan M, Sarwar M, Hossain MA, Wade JD, and Summers RJ (2014) Signalling profiles of $\mathrm{H} 3$ relaxin, $\mathrm{H} 2$ relaxin and $\mathrm{R} 3(\mathrm{~B} \Delta 23-27) \mathrm{R} / \mathrm{I} 5$ acting at the relaxin family peptide receptor 3 (RXFP3). Br J Pharmacol 171:2827-2841. 
Kohsaka T, Min G, Lukas G, Trupin S, Campbell ET, and Sherwood OD (1998) Identification of specific relaxin-binding cells in the human female. Biol Reprod 59: 991-999.

Kompa AR, Samuel CS, and Summers RJ (2002) Inotropic responses to human gene 2 (B29) relaxin in a rat model of myocardial infarction (MI): effect of pertussis toxin. Br J Pharmacol 137:710-718.

Kong RC, Petrie EJ, Mohanty B, Ling J, Lee JC, Gooley PR, and Bathgate RA (2013) The relaxin receptor (RXFP1) utilizes hydrophobic moieties on a signaling surface of its N-terminal low density lipoprotein class A module to mediate receptor activation. J Biol Chem 288:28138-28151.

Kong RC, Shilling PJ, Lobb DK, Gooley PR, and Bathgate RA (2010) Membrane receptors: structure and function of the relaxin family peptide receptors. Mol Cell Endocrinol 320:1-15.

Krajnc-Franken MA, van Disseldorp AJ, Koenders JE, Mosselman S, van Duin M, and Gossen JA (2004) Impaired nipple development and parturition in LGR7 knockout mice. $\mathrm{Mol}$ Cell Biol 24:687-696.

Kramer SM, Gibson UE, Fendly BM, Mohler MA, Drolet DW, and Johnston PD (1990) Increase in cyclic AMP levels by relaxin in newborn rhesus monkey uterus cell culture. In Vitro Cell Dev Biol 26:647-656.

Krantz JC Jr, Bryant HH, and Carr CJ (1950) The action of aqueous corpus luteum extract upon uterine activity. Surg Gynecol Obstet 90:372-375.

Kuei C, Sutton S, Bonaventure P, Pudiak C, Shelton J, Zhu J, Nepomuceno D, Wu J, Chen J, Kamme F, et al. (2007) R3(BDelta23 27)R/I5 chimeric peptide, a selective antagonist for GPCR135 and GPCR142 over relaxin receptor LGR7: in vitro and in vivo characterization. $J$ Biol Chem 282:25425-25435.

Kumagai J, Hsu SY, Matsumi H, Roh JS, Fu P, Wade JD, Bathgate RA, and Hsueh AJ (2002) INSL3/Leydig insulin-like peptide activates the LGR8 receptor important in testis descent. J Biol Chem 277:31283-31286.

Kupari M, Mikkola TS, Turto H, and Lommi J (2005) Is the pregnancy hormone relaxin an important player in human heart failure? Eur J Heart Fail 7:195-198.

Kuznetsova L, Plesneva S, Derjabina N, Omeljaniuk E, and Pertseva M (1999) On the mechanism of relaxin action: the involvement of adenylyl cyclase signalling system. Regul Pept 80:33-39.

Lee AB, Hwang JJ, Haab LM, Fields PA, and Sherwood OD (1992) Monoclonal antibodies specific for rat relaxin. VI. Passive immunization with monoclonal antibodies throughout the second half of pregnancy disrupts histological changes associated with cervical softening at parturition in rats. Endocrinology 130:2386-2391.

Lekgabe ED, Kiriazis H, Zhao C, Xu Q, Moore XL, Su Y, Bathgate RA, Du XJ, and Samuel CS (2005) Relaxin reverses cardiac and renal fibrosis in spontaneously hypertensive rats. Hypertension 46:412-418.

Li Y, Brookes ZL, and Kaufman S (2005) Acute and chronic effects of relaxin on vasoactivity, myogenic reactivity and compliance of the rat mesenteric arterial and venous vasculature. Regul Pept 132:41-46.

Lindheimer MD, Barron WM, and Davison JM (1989) Osmoregulation of thirst and vasopressin release in pregnancy. Am J Physiol 257:F159-F169.

Liu C, Chen J, Kuei C, Sutton S, Nepomuceno D, Bonaventure P, and Lovenberg TW (2005a) Relaxin-3/insulin-like peptide 5 chimeric peptide, a selective ligand for G protein-coupled receptor (GPCR)135 and GPCR142 over leucine-rich repeatcontaining G protein-coupled receptor 7. Mol Pharmacol 67:231-240.

Liu C, Chen J, Sutton S, Roland B, Kuei C, Farmer N, Sillard R, and Lovenberg TW (2003a) Identification of relaxin-3/INSL7 as a ligand for GPCR142. J Biol Chem 278:50765-50770.

Liu C, Eriste E, Sutton S, Chen J, Roland B, Kuei C, Farmer N, Jörnvall H, Sillard R, and Lovenberg TW (2003b) Identification of relaxin-3/INSL7 as an endogenous ligand for the orphan G-protein-coupled receptor GPCR135. J Biol Chem 278:50754-50764.

Liu C, Kuei C, Sutton S, Chen J, Bonaventure P, Wu J, Nepomuceno D, Kamme F, Tran DT, Zhu J, et al. (2005b) INSL5 is a high affinity specific agonist for GPCR142 (GPR100). J Biol Chem 280:292-300.

Loges S, Tinnefeld H, Metzner A, Jücker M, Butzal M, Bruweleit M, Fischer U, Draab E, Schuch G, O'-Farrel AM, et al. (2006) Downregulation of VEGF-A, STAT5 and AKT in acute myeloid leukemia blasts of patients treated with SU5416. Leuk Lymphoma 47:2601-2609.

Longo M, Jain V, Vedernikov YP, Garfield RE, and Saade GR (2003) Effects of recombinant human relaxin on pregnant rat uterine artery and myometrium in vitro. Am J Obstet Gynecol 188:1468-1474; discussion 1474-1466.

Lundgren S, Hjälm G, Hellman P, Ek B, Juhlin C, Rastad J, Klareskog L, Akerström $\mathrm{G}$, and Rask L (1994) A protein involved in calcium sensing of the human parathyroid and placental cytotrophoblast cells belongs to the LDL-receptor protein superfamily. Exp Cell Res 212:344-350.

Luttrell LM and Gesty-Palmer D (2010) Beyond desensitization: physiological relevance of arrestin-dependent signaling. Pharmacol Rev 62:305-330.

Ma JF, Liu L, Yang WJ, Zang LN, and Xi YM (2013a) RNAi-mediated knockdown of relaxin decreases in vitro proliferation and invasiveness of osteosarcoma MG-63 cells by inhibition of MMP-9. Eur Rev Med Pharmacol Sci 17:1102-1109.

Ma JF, Von Kalle M, Plautz Q, -M Xu F, Singh L, and Wang L (2013b) Relaxin promotes in vitro tumour growth, invasion and angiogenesis of human Saos-2 osteosarcoma cells by AKT/VEGF pathway. Eur Rev Med Pharmacol Sci 17:1345-1350.

Ma S, Bonaventure P, Ferraro T, Shen PJ, Burazin TC, Bathgate RA, Liu C, Tregear GW, Sutton SW, and Gundlach AL (2007a) Relaxin-3 in GABA projection neurons of nucleus incertus suggests widespread influence on forebrain circuits via G-proteincoupled receptor-135 in the rat. Neuroscience 144:165-190.

Ma S, Olucha-Bordonau FE, Hossain MA, Lin F, Kuei C, Liu C, Wade JD, Sutton SW Nuñez A, and Gundlach AL (2009a) Modulation of hippocampal theta oscillations and spatial memory by relaxin-3 neurons of the nucleus incertus. Learn Mem 16:730-742.

Ma S, Roozendaal B, Burazin TC, Tregear GW, McGaugh JL, and Gundlach AL (2005) Relaxin receptor activation in the basolateral amygdala impairs memory consolidation. Eur J Neurosci 22:2117-2122.

Ma S, Sang Q, Lanciego JL, and Gundlach AL (2009b) Localization of relaxin-3 in brain of Macaca fascicularis: identification of a nucleus incertus in primate. J Comp Neurol 517:856-872.
Ma S, Shen PJ, Burazin TC, Tregear GW, and Gundlach AL (2006) Comparative localization of leucine-rich repeat-containing G-protein-coupled receptor-7 (RXFP1) mRNA and $\left[{ }^{33} \mathrm{P}\right]$-relaxin binding sites in rat brain: restricted somatic co-expression a clue to relaxin action? Neuroscience 141:329-344.

Ma S, Shen PJ, Sang Q, Lanciego JL, and Gundlach AL (2009c) Distribution of relaxin-3 mRNA and immunoreactivity and RXFP3-binding sites in the brain of the macaque, Macaca fascicularis. Ann N Y Acad Sci 1160:256-258.

MacLennan AH, Grant P, and Bryant-Greenwood G (1995) hRLX-1. In vitro response of human and pig myometrium. J Reprod Med 40:703-706.

MacLennan AH, Grant P, Ness D, and Down A (1986a) Effect of porcine relaxin and progesterone on rat, pig and human myometrial activity in vitro. $J$ Reprod Med 31:43-49.

MacLennan AH, Green RC, Grant P, and Nicolson R (1986b) Ripening of the human cervix and induction of labor with intracervical purified porcine relaxin. Obstet Gynecol 68:598-601.

Mashima H, Ohno H, Yamada Y, Sakai T, and Ohnishi H (2013) INSL5 may be a unique marker of colorectal endocrine cells and neuroendocrine tumors. Biochem Biophys Res Commun 432:586-592.

Masini E, Bani D, Bello MG, Bigazzi M, Mannaioni PF, and Sacchi TB (1997) Relaxin counteracts myocardial damage induced by ischemia-reperfusion in isolated guinea pig hearts: evidence for an involvement of nitric oxide. Endocrinology 138:4713-4720.

Masini E, Nistri S, Vannacci A, Bani Sacchi T, Novelli A, and Bani D (2004) Relaxin inhibits the activation of human neutrophils: involvement of the nitric oxide pathway. Endocrinology 145:1106-1112.

Massicotte G, Parent A, and St-Louis J (1989) Blunted responses to vasoconstrictors in mesenteric vasculature but not in portal vein of spontaneously hypertensive rats treated with relaxin. Proc Soc Exp Biol Med 190:254-259.

Mathieu MN, Wade JD, Catimel B, Bond CP, Nice EC, Summers RJ, Otvos L Jr, and Tregear GW (2001) Synthesis, conformational studies and biological activity of N(alpha)-mono-biotinylated rat relaxin. J Pept Res 57:374-382.

Matsubara H (1998) Pathophysiological role of angiotensin II type 2 receptor in cardiovascular and renal diseases. Circ Res 83:1182-1191.

Matsumoto M, Kamohara M, Sugimoto T, Hidaka K, Takasaki J, Saito T, Okada M, Yamaguchi T, and Furuichi K (2000) The novel G-protein coupled receptor SALPR shares sequence similarity with somatostatin and angiotensin receptors. Gene $\mathbf{2 4 8}$ $183-189$

May LT, Leach K, Sexton PM, and Christopoulos A (2007) Allosteric modulation of G protein-coupled receptors. Annu Rev Pharmacol Toxicol 47:1-51.

McDonald GA, Sarkar P, Rennke H, Unemori E, Kalluri R, and Sukhatme VP (2003) Relaxin increases ubiquitin-dependent degradation of fibronectin in vitro and ameliorates renal fibrosis in vivo. Am J Physiol Renal Physiol 285:F59-F67.

McGowan BM, Minnion JS, Murphy KG, White NE, Roy D, Stanley SA, Dhillo WS, Gardiner JV, Ghatei MA, and Bloom SR (2010) Central and peripheral administration of human relaxin-2 to adult male rats inhibits food intake. Diabetes Obes Metab 12:1090-1096.

McGowan BM, Stanley SA, Smith KL, Minnion JS, Donovan J, Thompson EL, Patterson M, Connolly MM, Abbott CR, Small CJ, et al. (2006) Effects of acute and chronic relaxin-3 on food intake and energy expenditure in rats. Regul Pept 136:72-77.

McGowan BM, Stanley SA, Smith KL, White NE, Connolly MM, Thompson EL, Gardiner JV, Murphy KG, Ghatei MA, and Bloom SR (2005) Central relaxin-3 administration causes hyperphagia in male Wistar rats. Endocrinology 146:3295-3300.

McGuane JT, Danielson LA, Debrah JE, Rubin JP, Novak J, and Conrad KP (2011a) Angiogenic growth factors are new and essential players in the sustained relaxin vasodilatory pathway in rodents and humans. Hypertension 57:1151-1160.

McGuane JT, Debrah JE, Sautina L, Jarajapu YP, Novak J, Rubin JP, Grant MB, Segal M, and Conrad KP (2011b) Relaxin induces rapid dilation of rodent small renal and human subcutaneous arteries via PI3 kinase and nitric oxide. Endocrinology 152:2786-2796.

McKinley MJ, Allen AM, Burns P, Colvill LM, and Oldfield BJ (1998) Interaction of circulating hormones with the brain: the roles of the subfornical organ and the organum vasculosum of the lamina terminalis. Clin Exp Pharmacol Physiol Suppl 25:S61-S67.

McKinley MJ, Burns P, Colvill LM, Oldfield BJ, Wade JD, Weisinger RS, and Tregear GW (1997) Distribution of Fos immunoreactivity in the lamina terminalis and hypothalamus induced by centrally administered relaxin in conscious rats. $J$ Neuroendocrinol 9:431-437.

Millar LK, Reiny R, Yamamoto SY, Okazaki K, Webster L, and Bryant-Greenwood GD (2003) Relaxin causes proliferation of human amniotic epithelium by stimulation of insulin-like growth factor-II. Am J Obstet Gynecol 188:234-241.

Milligan G (2009) G protein-coupled receptor hetero-dimerization: contribution to pharmacology and function. Br J Pharmacol 158:5-14.

Minagawa I, Fukuda M, Ishige H, Kohriki H, Shibata M, Park EY, Kawarasaki T, and Kohsaka T (2012) Relaxin-like factor (RLF)/insulin-like peptide 3 (INSL3) is secreted from testicular Leydig cells as a monomeric protein comprising three domains B-C-A with full biological activity in boars. Biochem $J$ 441:265-273.

Miyamoto Y, Watanabe Y, and Tanaka M (2008) Developmental expression and serotonergic regulation of relaxin 3/INSL7 in the nucleus incertus of rat brain. Regul Pept 145:54-59.

Mookerjee I, Hewitson TD, Halls ML, Summers RJ, Mathai ML, Bathgate RA, Tregear GW, and Samuel CS (2009) Relaxin inhibits renal myofibroblast differentiation via RXFP1, the nitric oxide pathway, and Smad2. FASEB J 23:1219-1229.

Moore XL, Tan SL, Lo CY, Fang L, Su YD, Gao XM, Woodcock EA, Summers RJ, Tregear GW, Bathgate RA, et al. (2007) Relaxin antagonizes hypertrophy and apoptosis in neonatal rat cardiomyocytes. Endocrinology 148:1582-1589.

Morikawa Y, Ueyama E, and Senba E (2004) Fasting-induced activation of mitogenactivated protein kinases (ERK/p38) in the mouse hypothalamus. $J$ Neuroendocrinol 16:105-112.

Muda M, He C, Martini PG, Ferraro T, Layfield S, Taylor D, Chevrier C, Schweickhardt R, Kelton C, Ryan PL, et al. (2005) Splice variants of the relaxin and INSL3 receptors reveal unanticipated molecular complexity. Mol Hum Reprod 11:591-600. 
Munro J, Skrobot O, Sanyoura M, Kay V, Susce MT, Glaser PE, de Leon J, Blakemore AI, and Arranz MJ (2012) Relaxin polymorphisms associated with metabolic disturbance in patients treated with antipsychotics. J Psychopharmacol 26:374-379.

Nakanishi H, Brewer KA, and Exton JH (1993) Activation of the zeta isozyme of protein kinase $\mathrm{C}$ by phosphatidylinositol 3,4,5-trisphosphate. $J$ Biol Chem 268: $13-16$.

Nef S and Parada LF (1999) Cryptorchidism in mice mutant for Insl3. Nat Genet 22: 295-299.

Neschadim A, Pritzker LB, Pritzker KP, Branch DR, Summerlee AJ, Trachtenberg J, and Silvertown JD (2014) Relaxin receptor antagonist AT-001 synergizes with docetaxel in androgen-independent prostate xenografts. Endocr Relat Cancer 21: $459-471$.

Nguyen BT and Dessauer CW (2005a) Relaxin stimulates cAMP production in MCF-7 cells upon overexpression of type V adenylyl cyclase. Ann N Y Acad Sci 1041: 296-299.

Nguyen BT and Dessauer CW (2005b) Relaxin stimulates protein kinase C $\zeta$ translocation requirement for cyclic adenosine 3', $5^{\prime}$-monophosphate production. Mol Endocrinol 19: $1012-1023$

Nguyen BT, Yang L, Sanborn BM, and Dessauer CW (2003) Phosphoinositide 3-kinase activity is required for biphasic stimulation of cyclic adenosine $3^{\prime}, 5^{\prime}$ monophosphate by relaxin. Mol Endocrinol 17:1075-1084.

Nistri S and Bani D (2003) Relaxin receptors and nitric oxide synthases: search for the missing link. Reprod Biol Endocrinol 1:5.

Novak J, Danielson LA, Kerchner LJ, Sherwood OD, Ramirez RJ, Moalli PA, and Conrad KP (2001) Relaxin is essential for renal vasodilation during pregnancy in conscious rats. $J$ Clin Invest 107:1469-1475

Novak J, Parry LJ, Matthews JE, Kerchner LJ, Indovina K, Hanley-Yanez K, Doty $\mathrm{KD}$, Debrah DO, Shroff SG, and Conrad KP (2006) Evidence for local relaxin ligand-receptor expression and function in arteries. FASEB J 20:2352-2362.

Novak J, Ramirez RJ, Gandley RE, Sherwood OD, and Conrad KP (2002) Myogenic reactivity is reduced in small renal arteries isolated from relaxin-treated rats. Am $J$ Physiol Regul Integr Comp Physiol 283:R349-R355.

Nuñez A, Cervera-Ferri A, Olucha-Bordonau F, Ruiz-Torner A, and Teruel V (2006) Nucleus incertus contribution to hippocampal theta rhythm generation. Eur $J$ Neurosci 23:2731-2738.

O'Byrne EM, Carriere BT, Sorensen L, Segaloff A, Schwabe C, and Steinetz BG (1978) Plasma immunoreactive relaxin levels in pregnant and nonpregnant women. J Clin Endocrinol Metab 47:1106-1110.

O'Connor WB, Cain GD, and Zarrow MX (1966) Elongation of the interpubic ligament in the little brown bat (Myotis lucifugus). Proc Soc Exp Biol Med 123:935-937.

Olefsky JM, Saekow M, and Kroc RL (1982) Potentiation of insulin binding and insulin action by purified porcine relaxin. Ann N Y Acad Sci 380:200-216.

Olucha-Bordonau FE, Teruel V, Barcia-González J, Ruiz-Torner A, Valverde-Navarro AA, and Martínez-Soriano F (2003) Cytoarchitecture and efferent projections of the nucleus incertus of the rat. J Comp Neurol 464:62-97.

Ono Y, Fujii T, Ogita K, Kikkawa U, Igarashi K, and Nishizuka Y (1989) Protein kinase C zeta subspecies from rat brain: its structure, expression, and properties. Proc Natl Acad Sci USA 86:3099-3103.

Orencole SF and Dinarello CA (1989) Characterization of a subclone (D10S) of the D10.G4.1 helper T-cell line which proliferates to attomolar concentrations of interleukin-1 in the absence of mitogens. Cytokine 1:14-22.

Osa T, Inoue H, and Okabe K (1991) Effects of porcine relaxin on contraction, membrane response and cyclic AMP content in rat myometrium in comparison with the effects of isoprenaline and forskolin. Br J Pharmacol 104:950-960.

Osheroff PL, Cronin MJ, and Lofgren JA (1992) Relaxin binding in the rat heart atrium. Proc Natl Acad Sci USA 89:2384-2388.

Osheroff PL and Ho WH (1993) Expression of relaxin mRNA and relaxin receptors in postnatal and adult rat brains and hearts. Localization and developmental patterns. J Biol Chem 268:15193-15199.

Overbeek PA, Gorlov IP, Sutherland RW, Houston JB, Harrison WR, Boettger-Tong HL, Bishop CE, and Agoulnik AI (2001) A transgenic insertion causing cryptorchidism in mice. Genesis 30:26-35.

Palejwala S, Stein D, Wojtczuk A, Weiss G, and Goldsmith LT (1998) Demonstration of a relaxin receptor and relaxin-stimulated tyrosine phosphorylation in human lower uterine segment fibroblasts. Endocrinology 139:1208-1212.

Pan HZ, Dong AB, Wang L, Tan SS, Yang Q, Tong XY, Liang J, and Wang JR (2013) Significance of relaxin-2 expression in hepatocellular carcinoma: relation with clinicopathological parameters. Eur Rev Med Pharmacol Sci 17:1095-1101.

Parikh A, Patel D, McTiernan CF, Xiang W, Haney J, Yang L, Lin B, Kaplan AD, Bett GC, Rasmusson RL, et al. (2013) Relaxin suppresses atrial fibrillation by reversing fibrosis and myocyte hypertrophy and increasing conduction velocity and sodium current in spontaneously hypertensive rat hearts. Circ Res 113:313-321.

Park JI, Semyonov J, Yi W, Chang CL, and Hsu SY (2008) Regulation of receptor signaling by relaxin A chain motifs: derivation of pan-specific and LGR7-specific human relaxin analogs. J Biol Chem 283:32099-32109.

Parsell DA, Mak JY, Amento EP, and Unemori EN (1996) Relaxin binds to and elicits a response from cells of the human monocytic cell line, THP-1. J Biol Chem 271:27936-27941.

Pathirana IN, Kawate N, Büllesbach EE, Takahashi M, Hatoya S, Inaba T, and Tamada $H$ (2012) Insulin-like peptide 3 stimulates testosterone secretion in mouse Leydig cells via cAMP pathway. Regul Pept 178:102-106.

Pepe A, Ferlin A, Gianesello L, Facciolli A, Agoulnik AI, and Foresta C (2009) INSL3 plays a role in the balance between bone formation and resorption. Ann N Y Acad Sci 1160:219-220.

Perna AM, Masini E, Nistri S, Briganti V, Chiappini L, Stefano P, Bigazzi M, Pieroni C, Bani Sacchi T, and Bani D (2005) Novel drug development opportunity for relaxin in acute myocardial infarction: evidences from a swine model. FASEB J 19 1525-1527.

Petersen LK, Svane D, Uldbjerg N, and Forman A (1991) Effects of human relaxin on isolated rat and human myometrium and uteroplacental arteries. Obstet Gynecol 78:757-762.
Piedras-Rentería ES, Sherwood OD, and Best PM (1997a) Effects of relaxin on rat atrial myocytes. I. Inhibition of I(to) via PKA-dependent phosphorylation. Am J Physiol 272:H1791-H1797.

Piedras-Rentería ES, Sherwood OD, and Best PM (1997b) Effects of relaxin on rat atrial myocytes. II. Increased calcium influx derived from action potential prolongation. Am J Physiol 272:H1798-H1803.

Pietilä EM, Tuusa JT, Apaja PM, Aatsinki JT, Hakalahti AE, Rajaniemi HJ, and Petäjä-Repo UE (2005) Inefficient maturation of the rat luteinizing hormone receptor. A putative way to regulate receptor numbers at the cell surface. J Biol Chem 280:26622-26629.

Pin JP, Neubig R, Bouvier M, Devi L, Filizola M, Javitch JA, Lohse MJ, Milligan G, Palczewski K, Parmentier M, et al. (2007) International Union of Basic and Clinical Pharmacology. LXVII. Recommendations for the recognition and nomenclature of $\mathrm{G}$ protein-coupled receptor heteromultimers. Pharmacol Rev 59:5-13.

Pini A, Shemesh R, Samuel CS, Bathgate RA, Zauberman A, Hermesh C, Wool A Bani D, and Rotman G (2010) Prevention of bleomycin-induced pulmonary fibrosis by a novel antifibrotic peptide with relaxin-like activity. J Pharmacol Exp Ther 335:589-599.

Price MA, Cruzalegui FH, and Treisman R (1996) The p38 and ERK MAP kinase pathways cooperate to activate Ternary Complex Factors and c-fos transcription in response to UV light. EMBO J 15:6552-6563.

Pusch W, Balvers M, and Ivell R (1996) Molecular cloning and expression of the relaxin-like factor from the mouse testis. Endocrinology 137:3009-3013.

Quaggin SE, Schwartz L, Cui S, Igarashi P, Deimling J, Post M, and Rossant J (1999) The basic-helix-loop-helix protein pod1 is critically important for kidney and lung organogenesis. Development 126:5771-5783.

Quintana J, Hipkin RW, and Ascoli M (1993) A polyclonal antibody to a synthetic peptide derived from the rat follicle-stimulating hormone receptor reveals the recombinant receptor as a 74-kilodalton protein. Endocrinology 133:2098-2104.

Radestock Y, Hoang-Vu C, and Hombach-Klonisch S (2008) Relaxin reduces xenograft tumour growth of human MDA-MB-231 breast cancer cells. Breast Cancer Res 10:R71.

Ren P, Yu ZT, Xiu L, Wang M, and Liu HM (2013) Elevated serum levels of human relaxin-2 in patients with esophageal squamous cell carcinoma. World J Gastroenterol 19:2412-2418.

Ren Q, Kurose H, Lefkowitz RJ, and Cotecchia S (1993) Constitutively active mutants of the alpha 2-adrenergic receptor. J Biol Chem 268:16483-16487.

Roche PJ, Butkus A, Wintour EM, and Tregear G (1996) Structure and expression of Leydig insulin-like peptide mRNA in the sheep. Mol Cell Endocrinol 121:171-177. Rosengren KJ, Bathgate RA, Craik DJ, Daly NL, Haugaard-Jönsson LM, Hossain MA, and Wade JD (2009) Structural insights into the function of relaxins. Ann NY Acad Sci 1160:20-26.

Rosengren KJ, Lin F, Bathgate RA, Tregear GW, Daly NL, Wade JD, and Craik DJ (2006a) Solution structure and novel insights into the determinants of the receptor specificity of human relaxin-3. J Biol Chem 281:5845-5851.

Rosengren KJ, Zhang S, Lin F, Daly NL, Scott DJ, Hughes RA, Bathgate RA, Craik DJ, and Wade JD (2006b) Solution structure and characterization of the LGR8 receptor binding surface of insulin-like peptide 3. J Biol Chem 281:28287-28295.

Roux PP and Blenis J (2004) ERK and p38 MAPK-activated protein kinases: a family of protein kinases with diverse biological functions. Microbiol Mol Biol Rev 68: 320-344.

Ryan PJ, Büchler E, Shabanpoor F, Hossain MA, Wade JD, Lawrence AJ, and Gundlach AL (2013a) Central relaxin-3 receptor (RXFP3) activation decreases anxiety- and depressive-like behaviours in the rat. Behav Brain Res 244:142-151.

Ryan PJ, Kastman HE, Krstew EV, Rosengren KJ, Hossain MA, Churilov L, Wade JD, Gundlach AL, and Lawrence AJ (2013b) Relaxin-3/RXFP3 system regulates alcohol-seeking. Proc Natl Acad Sci USA 110:20789-20794.

Sadana R and Dessauer CW (2009) Physiological roles for G protein-regulated adenylyl cyclase isoforms: insights from knockout and overexpression studies. Neurosignals 17:5-22.

Samuel CS, Cendrawan S, Gao XM, Ming Z, Zhao C, Kiriazis H, Xu Q, Tregear GW, Bathgate RA, and Du XJ (2011) Relaxin remodels fibrotic healing following myocardial infarction. Lab Invest 91:675-690.

Samuel CS, Hewitson TD, Zhang Y, and Kelly DJ (2008) Relaxin ameliorates fibrosis in experimental diabetic cardiomyopathy. Endocrinology 149:3286-3293.

Samuel CS, Lin F, Hossain MA, Zhao C, Ferraro T, Bathgate RA, Tregear GW, and Wade JD (2007) Improved chemical synthesis and demonstration of the relaxin receptor binding affinity and biological activity of mouse relaxin. Biochemistry 46 $5374-5381$

Samuel CS, Tian H, Zhao L, and Amento EP (2003a) Relaxin is a key mediator of prostate growth and male reproductive tract development. Lab Invest 83:1055-1067. Samuel CS, Unemori EN, Mookerjee I, Bathgate RA, Layfield SL, Mak J, Tregear GW, and Du XJ (2004a) Relaxin modulates cardiac fibroblast proliferation, differentiation, and collagen production and reverses cardiac fibrosis in vivo. Endocrinology 145:4125-4133.

Samuel CS, Zhao C, Bathgate RA, Bond CP, Burton MD, Parry LJ, Summers RJ, Tang ML, Amento EP, and Tregear GW (2003b) Relaxin deficiency in mice is associated with an age-related progression of pulmonary fibrosis. FASEB J 17:121-123.

Samuel CS, Zhao C, Bond CP, Hewitson TD, Amento EP, and Summers RJ (2004b) Relaxin-1-deficient mice develop an age-related progression of renal fibrosis. Kidney Int 65:2054-2064.

Samuel CS, Zhao C, Yang Q, Wang H, Tian H, Tregear GW, and Amento EP (2005) The relaxin gene knockout mouse: a model of progressive scleroderma. J Invest Dermatol 125:692-699.

Sanborn BM, Kuo HS, Weisbrodt NW, and Sherwood OD (1980) The interaction of relaxin with the rat uterus. I. Effect on cyclic nucleotide levels and spontaneous contractile activity. Endocrinology 106:1210-1215.

Sánchez-Blázquez P and Garzón J (1995) $\alpha$ N-acetyl- $\beta$-endorphin-(1-31) disrupts the diminishing effect of mastoparan on opioid- and clonidine-evoked supraspinal antinociception in mice. J Pharmacol Exp Ther 273:787-792. 
Sarwar M, Samuel CS, Bathgate RA, Stewart DR, and Summers RJ (2015) Serelaxinmediated signal transduction in human vascular cells: bell-shaped concentrationresponse curves reflect differential coupling to G proteins. $\mathrm{Br} J$ Pharmacol 172: $1005-1019$.

Sasaguri K, Kikuchi M, Hori N, Yuyama N, Onozuka M, and Sato S (2005) Suppression of stress immobilization-induced phosphorylation of ERK 1/2 by biting in the rat hypothalamic paraventricular nucleus. Neurosci Lett 383:160-164.

Sassoli C, Chellini F, Pini A, Tani A, Nistri S, Nosi D, Zecchi-Orlandini S, Bani D, and Formigli L (2013) Relaxin prevents cardiac fibroblast-myofibroblast transition via notch-1-mediated inhibition of TGF-3/Smad3 signaling. PLoS ONE 8:e63896.

Savoia C, Ebrahimian T, He Y, Gratton JP, Schiffrin EL, and Touyz RM (2006) Angiotensin II/AT2 receptor-induced vasodilation in stroke-prone spontaneously hypertensive rats involves nitric oxide and cGMP-dependent protein kinase. J Hypertens 24:2417-2422.

Schink W and Struck H (1968) [Relaxin in the Allen-Doisy test]. Zentralbl Gynakol 90:675-678.

Schöndorf T, Lübben G, Hoopmann M, Borchert M, Forst T, Hohberg C, Löbig M, Armbruster FP, Roth W, Grabellus M, et al. (2007) Relaxin expression correlates significantly with serum fibrinogen variation in response to antidiabetic treatment in women with type 2 diabetes mellitus. Gynecol Endocrinol 23:356-360.

Schwabe C and Büllesbach EE (1994) Relaxin: structures, functions, promises, and nonevolution. FASEB J 8:1152-1160.

Schwabe C and McDonald JK (1977) Relaxin: a disulfide homolog of insulin. Science 197:914-915.

Scott DJ, Fu P, Shen PJ, Gundlach A, Layfield S, Riesewijk A, Tomiyama H, Hutson JM, Tregear GW, and Bathgate RA (2005a) Characterization of the rat INSL3 receptor. Ann N Y Acad Sci 1041:13-16.

Scott DJ, Layfield S, Riesewijk A, Morita H, Tregear GW, and Bathgate RA (2005b) Characterization of the mouse and rat relaxin receptors. Ann N Y Acad Sci 1041: $8-12$

Scott DJ, Layfield S, Yan Y, Sudo S, Hsueh AJ, Tregear GW, and Bathgate RA (2006) Characterization of novel splice variants of LGR7 and LGR8 reveals that receptor signaling is mediated by their unique low density lipoprotein class A modules. $J$ Biol Chem 281:34942-34954.

Scott DJ, Rosengren KJ, and Bathgate RA (2012) The different ligand-binding modes of relaxin family peptide receptors RXFP1 and RXFP2. Mol Endocrinol 26 1896-1906.

Scott DJ, Tregear GW, and Bathgate RA (2005c) LGR7-truncate is a splice variant of the relaxin receptor LGR7 and is a relaxin antagonist in vitro. Ann N Y Acad Sci 1041:22-26.

Scott DJ, Wilkinson TN, Zhang S, Ferraro T, Wade JD, Tregear GW, and Bathgate RA (2007) Defining the LGR8 residues involved in binding insulin-like peptide 3. Mol Endocrinol 21:1699-1712.

Sedaghat K, Shen PJ, Finkelstein DI, Henderson JM, and Gundlach AL (2008) Leucine-rich repeat-containing G-protein-coupled receptor 8 in the rat brain: Enrichment in thalamic neurons and their efferent projections. Neuroscience $\mathbf{1 5 6}$ 319-333.

Seibold JR, Korn JH, Simms R, Clements PJ, Moreland LW, Mayes MD, Furst DE, Rothfield N, Steen V, Weisman M, et al. (2000) Recombinant human relaxin in the treatment of scleroderma. A randomized, double-blind, placebo-controlled trial. Ann Intern Med 132:871-879.

Shabanpoor F, Akhter Hossain M, Ryan PJ, Belgi A, Layfield S, Kocan M, Zhang S, Samuel CS, Gundlach AL, Bathgate RA, et al. (2012) Minimization of human relaxin-3 leading to high-affinity analogues with increased selectivity for relaxinfamily peptide 3 receptor (RXFP3) over RXFP1. J Med Chem 55:1671-1681.

Shabanpoor F, Bathgate RA, Hossain MA, Giannakis E, Wade JD, and Hughes RA (2007) Design, synthesis and pharmacological evaluation of cyclic mimetics of the insulin-like peptide 3 (INSL3) B-chain. J Pept Sci 13:113-120.

Shabanpoor F, Hughes RA, Zhang S, Bathgate RA, Layfield S, Hossain MA, Tregear GW, Separovic F, and Wade JD (2010) Effect of helix-promoting strategies on the biological activity of novel analogues of the B-chain of INSL3. Amino Acids 38 121-131.

Shabanpoor F, Zhang S, Hughes RA, Hossain MA, Layfield S, Ferraro T, Bathgate RA, Separovic F, and Wade JD (2011) Design and development of analogues of dimers of insulin-like peptide 3 B-chain as high-affinity antagonists of the RXFP2 receptor. Biopolymers 96:81-87.

Shaw EE, Wood P, Kulpa J, Yang FH, Summerlee AJ, and Pyle WG (2009) Relaxin alters cardiac myofilament function through a PKC-dependent pathway. Am J Physiol Heart Circ Physiol 297:H29-H36.

Shemesh R, Hermesh C, Toporik A, Levine Z, Novik A, Wool A, Kliger Y, Rosenberg A, Bathgate RA, and Cohen Y (2009) Activation of relaxin-related receptors by short, linear peptides derived from a collagen-containing precursor. Ann N Y Acad Sci 1160:78-86.

Shemesh R, Toporik A, Levine Z, Hecht I, Rotman G, Wool A, Dahary D, Gofer E, Kliger Y, Soffer MA, et al. (2008) Discovery and validation of novel peptide agonists for G-protein-coupled receptors. J Biol Chem 283:34643-34649.

Shen CP, Tsimberg Y, Salvadore C, and Meller E (2004) Activation of Erk and JNK MAPK pathways by acute swim stress in rat brain regions. BMC Neurosci 5:36.

Shen PJ, Fu P, Phelan KD, Scott DJ, Layfield S, Tregear GW, Bathgate RA, and Gundlach AL (2005) Restricted expression of LGR8 in intralaminar thalamic nuclei of rat brain suggests a role in sensorimotor systems. Ann N Y Acad Sci 1041:510-515.

Sherwood OD (1994) Relaxin, in The Physiology of Reproduction (Knobil E and Neill JD eds) pp 861-1009, Raven Press, New York.

Sherwood OD (2004) Relaxin's physiological roles and other diverse actions. Endocr Rev 25:205-234.

Sherwood OD, Crnekovic VE, Gordon WL, and Rutherford JE (1980) Radioimmunoassay of relaxin throughout pregnancy and during parturition in the rat. Endocrinology 107:691-698.

Shpakov AO, Gur'yanov IA, Kuznetsova LA, Plesneva SA, Shpakova EA, Vlasov GP, and Pertseva MN (2007) Studies of the molecular mechanisms of action of relaxin on the adenylyl cyclase signaling system using synthetic peptides derived from the LGR7 relaxin receptor. Neurosci Behav Physiol 37:705-714.

Shymko RM, De Meyts P, and Thomas R (1997) Logical analysis of timing-dependent receptor signalling specificity: application to the insulin receptor metabolic and mitogenic signalling pathways. Biochem $J$ 326:463-469.

Silvertown JD, Ng J, Sato T, Summerlee AJ, and Medin JA (2006) H2 relaxin overexpression increases in vivo prostate xenograft tumor growth and angiogenesis. Int $J$ Cancer 118:62-73.

Silvertown JD, Summerlee AJ, and Klonisch T (2003) Relaxin-like peptides in cancer. Int $J$ Cancer 107:513-519.

Silvertown JD, Symes JC, Neschadim A, Nonaka T, Kao JC, Summerlee AJ, and Medin JA (2007) Analog of $\mathrm{H} 2$ relaxin exhibits antagonistic properties and impairs prostate tumor growth. FASEB J 21:754-765.

Singh S and Bennett RG (2010) Relaxin signaling activates peroxisome proliferatoractivated receptor gamma. Mol Cell Endocrinol 315:239-245.

Siqin MI, Minagawa I, Okuno M, Yamada K, Sugawara Y, Nagura Y, Hamano K, Park EY, Sasada H, and Kohsaka T (2013) The active form of goat insulin-like peptide 3 (INSL3) is a single-chain structure comprising three domains B-C-A, constitutively expressed and secreted by testicular Leydig cells. Biol Chem 394: 1181-1194.

Siragy HM and Carey RM (1997) The subtype 2 (AT2) angiotensin receptor mediates renal production of nitric oxide in conscious rats. J Clin Invest 100:264-269.

Skroblin P, Grossmann S, Schäfer G, Rosenthal W, and Klussmann E (2010) Mechanisms of protein kinase A anchoring. Int Rev Cell Mol Biol 283:235-330.

Smith CM, Chua BE, Zhang C, Walker AW, Haidar M, Hawkes D, Shabanpoor F, Hossain MA, Wade JD, Rosengren KJ, et al. (2014) Central injection of relaxin-3 receptor (RXFP3) antagonist peptides reduces motivated food seeking and consumption in C57BL/6J mice. Behav Brain Res 268:117-126.

Smith CM, Lawrence AJ, Sutton SW, and Gundlach AL (2009) Behavioral phenotyping of mixed background (129S5:B6) relaxin-3 knockout mice. Ann N Y Acad Sci 1160:236-241.

Smith CM, Ryan PJ, Hosken IT, Ma S, and Gundlach AL (2011) Relaxin-3 systems in the brain-the first 10 years. J Chem Neuroanat 42:262-275.

Smith CM, Shen PJ, Banerjee A, Bonaventure P, Ma S, Bathgate RA, Sutton SW, and Gundlach AL (2010) Distribution of relaxin-3 and RXFP3 within arousal, stress, affective, and cognitive circuits of mouse brain. J Comp Neurol 518:4016-4045.

Smith MC, Danielson LA, Conrad KP, and Davison JM (2006a) Influence of recombinant human relaxin on renal hemodynamics in healthy volunteers. $J \mathrm{Am}$ Soc Nephrol 17:3192-3197.

Smith MC, Murdoch AP, Danielson LA, Conrad KP, and Davison JM (2006b) Relaxin has a role in establishing a renal response in pregnancy. Fertil Steril 86:253-255.

Sokol RZ, Wang XS, Lechago J, Johnston PD, and Swerdloff RS (1989) Immunohistochemical localization of relaxin in human prostate. J Histochem Cytochem 37: $1253-1255$

Spanel-Borowski K, Schäfer I, Zimmermann S, Engel W, and Adham IM (2001) Increase in final stages of follicular atresia and premature decay of corpora lutea in Insl3-deficient mice. Mol Reprod Dev 58:281-286.

Standaert ML, Bandyopadhyay G, Kanoh Y, Sajan MP, and Farese RV (2001) Insulin and PIP3 activate PKC-zeta by mechanisms that are both dependent and independent of phosphorylation of activation loop (T410) and autophosphorylation (T560) sites. Biochemistry 40:249-255.

Stanley KK, Kocher HP, Luzio JP, Jackson P, and Tschopp J (1985) The sequence and topology of human complement component C9. EMBO J 4:375-382.

Steinetz BG, Beach VL, Blye RP, and Kroc RL (1957) Changes in the composition of the rat uterus following a single injection of relaxin. Endocrinology 61:287-292.

Steinetz BG, O'Byrne EM, Butler MC, and Hickman LB (1983) Hormonal regulation of the connective tissue of the symphysis pubis, in Biology of Relaxin and Its Role in the Human (Bigazzi M, Greenwood FC, and Gasparri F eds) pp 71-92, Excerpta Medica, Amsterdam

Steinetz BG, Whitaker PG, and Edwards JR (1992) Maternal relaxin concentrations in diabetic pregnancy. Lancet 340:752-755.

Stewart DR, Celniker AC, Taylor CA Jr, Cragun JR, Overstreet JW, and Lasley BL (1990) Relaxin in the peri-implantation period. J Clin Endocrinol Metab 70:1771-1773. St-Louis J and Massicotte G (1985) Chronic decrease of blood pressure by rat relaxin in spontaneously hypertensive rats. Life Sci 37:1351-1357.

Stöhr H, Berger C, Fröhlich S, and Weber BH (2002) A novel gene encoding a putative transmembrane protein with two extracellular CUB domains and a lowdensity lipoprotein class A module: isolation of alternatively spliced isoforms in retina and brain. Gene 286:223-231.

Sudo S, Kumagai J, Nishi S, Layfield S, Ferraro T, Bathgate RA, and Hsueh AJ (2003) H3 relaxin is a specific ligand for LGR7 and activates the receptor by interacting with both the ectodomain and the exoloop 2. J Biol Chem 278:7855-7862.

Summerlee AJ, Hornsby DJ, and Ramsey DG (1998a) The dipsogenic effects of rat relaxin: The effect of photoperiod and the potential role of relaxin on drinking in pregnancy. Endocrinology 139:2322-2328.

Summerlee AJ, Ramsey DG, and Poterski RS (1998b) Neutralization of relaxin within the brain affects the timing of birth in rats. Endocrinology 139:479-484.

Sunn N, Egli M, Burazin TC, Burns P, Colvill L, Davern P, Denton DA, Oldfield BJ, Weisinger RS, Rauch M, et al. (2002) Circulating relaxin acts on subfornical organ neurons to stimulate water drinking in the rat. Proc Natl Acad Sci USA 99. $1701-1706$

Sunn N, McKinley MJ, and Oldfield BJ (2001) Identification of efferent neura pathways from the lamina terminalis activated by blood-borne relaxin. $J$ Neuroendocrinol 13:432-437.

Sutton RE, Koob GF, Le Moal M, Rivier J, and Vale W (1982) Corticotropin releasing factor produces behavioural activation in rats. Nature 297:331-333.

Sutton SW, Bonaventure P, Kuei C, Roland B, Chen J, Nepomuceno D, Lovenberg TW, and Liu C (2004) Distribution of G-protein-coupled receptor (GPCR)135 binding sites and receptor mRNA in the rat brain suggests a role for relaxin-3 in neuroendocrine and sensory processing. Neuroendocrinology 80:298-307. 
Sutton SW, Shelton J, Smith C, Williams J, Yun S, Motley T, Kuei C, Bonaventure P, Gundlach A, Liu C, et al. (2009) Metabolic and neuroendocrine responses to RXFP3 modulation in the central nervous system. Ann N Y Acad Sci 1160:242-249.

Suzuki K, Nakabayashi K, Yamada AY, Lodhi RS, Hazama R, Ebina Y, and Yamada H (2012) Recombinant H2 relaxin inhibits apoptosis and induces cell proliferation in cultured leiomyoma cells without affecting those in cultured normal myometrial cells. Fertil Steril 97:734-741.

Svendsen AM, Vrecl M, Ellis TM, Heding A, Kristensen JB, Wade JD, Bathgate RA, De Meyts P, and Nøhr J (2008a) Cooperative binding of insulin-like Peptide 3 to a dimeric relaxin family peptide receptor 2. Endocrinology 149:1113-1120.

Svendsen AM, Zalesko A, Kønig J, Vrecl M, Heding A, Kristensen JB, Wade JD, Bathgate RA, De Meyts P, and Nøhr J (2008b) Negative cooperativity in H2 relaxin binding to a dimeric relaxin family peptide receptor 1. Mol Cell Endocrinol 296: $10-17$.

Szepietowska B, Gorska M, and Szelachowska M (2008) Plasma relaxin concentration is related to beta-cell function and insulin sensitivity in women with type 2 diabetes mellitus. Diabetes Res Clin Pract 79:e1-e3.

Szydlarska D, Grzesiuk W, Trybuch A, Kondracka A, Kowalik I, and Bar-Andziak E (2012) Insulin-like factor 3 - a new hormone related to polycystic ovary syndrome? Endokrynol Pol 63:356-361.

Tan YY, Dawson NF, Kompa AR, Bond CP, Claasz A, Wade JD, Tregear GW, and Summers RJ (2002) Structural requirements for the interaction of sheep insulinlike factor 3 with relaxin receptors in rat atria. Eur J Pharmacol 457:153-160.

Tan YY, Wade JD, Tregear GW, and Summers RJ (1998) Comparison of relaxin receptors in rat isolated atria and uterus by use of synthetic and native relaxin analogues. Br J Pharmacol 123:762-770.

Tan YY, Wade JD, Tregear GW, and Summers RJ (1999) Quantitative autoradiographic studies of relaxin binding in rat atria, uterus and cerebral cortex: characterization and effects of oestrogen treatment. $\mathrm{Br} J$ Pharmacol 127:91-98.

Tanaka M, Iijima N, Miyamoto Y, Fukusumi S, Itoh Y, Ozawa H, and Ibata Y (2005) Neurons expressing relaxin 3/INSL 7 in the nucleus incertus respond to stress. Eur $J$ Neurosci 21:1659-1670.

Tao Y-X, Johnson NB, and Segaloff DL (2004) Constitutive and agonist-dependent self-association of the cell surface human lutropin receptor. $J$ Biol Chem $\mathbf{2 7 9}$ $5904-5914$

Tashima LS, Hieber AD, Greenwood FC, and Bryant-Greenwood GD (1995) The human Leydig insulin-like (hLEY I-L) gene is expressed in the corpus luteum and trophoblast. J Clin Endocrinol Metab 80:707-710.

Tashima LS, Mazoujian G, and Bryant-Greenwood GD (1994) Human relaxins in normal, benign and neoplastic breast tissue. J Mol Endocrinol 12:351-364.

Teerlink JR, Cotter G, Davison BA, Felker GM, Filippatos G, Greenberg BH, Ponikowski $\mathrm{P}$, Unemori E, Voors AA, Adams KF Jr, et al. RELAXin in Acute Heart Failure (RELAX-AHF) Investigators (2013) Serelaxin, recombinant human relaxin-2, for treatment of acute heart failure (RELAX-AHF): a randomised, placebo-controlled trial. Lancet 381:29-39.

Teerlink JR, Metra M, Felker GM, Ponikowski P, Voors AA, Weatherley BD, Marmor A, Katz A, Grzybowski J, Unemori E, et al. (2009) Relaxin for the treatment of patients with acute heart failure (Pre-RELAX-AHF): a multicentre, randomised, placebo-controlled, parallel-group, dose-finding phase IIb study. Lancet 373:1429-1439.

Teichman SL, Unemori E, Dschietzig T, Conrad K, Voors AA, Teerlink JR, Felker GM, Metra M, and Cotter G (2009) Relaxin, a pleiotropic vasodilator for the treatment of heart failure. Heart Fail Rev 14:321-329.

Telgmann R and Gellersen B (1998) Marker genes of decidualization: activation of the decidual prolactin gene. Hum Reprod Update 4:472-479.

Thanasupawat T, Hammje K, Adham I, Ghia JE, Del Bigio MR, Krcek J, Hoang-Vu C, Klonisch T, and Hombach-Klonisch S (2013) INSL5 is a novel marker for human enteroendocrine cells of the large intestine and neuroendocrine tumours. Oncol Rep 29:149-154.

Thomas GR and Vandlen R (1993) The purely chronotropic effects of relaxin in the rat isolated heart. J Pharm Pharmacol 45:927-928.

Thompson VC, Morris TG, Cochrane DR, Cavanagh J, Wafa LA, Hamilton T, Wang S, Fazli L, Gleave ME, and Nelson CC (2006) Relaxin becomes upregulated during prostate cancer progression to androgen independence and is negatively regulated by androgens. Prostate 66:1698-1709.

Tomboc M, Lee PA, Mitwally MF, Schneck FX, Bellinger M, and Witchel SF (2000) Insulin-like 3/relaxin-like factor gene mutations are associated with cryptorchidism. $J$ Clin Endocrinol Metab 85:4013-4018.

Toth M, Taskinen P, and Ruskoaho H (1996) Relaxin stimulates atrial natriuretic peptide secretion in perfused rat heart. $J$ Endocrinol 150:487-495.

Ueda H, Inoue M, Yoshida A, Mizuno K, Yamamoto H, Maruo J, Matsuno K, and Mita S (2001) Metabotropic neurosteroid/sigma-receptor involved in stimulation of nociceptor endings of mice. J Pharmacol Exp Ther 298:703-710.

Unemori EN, Erikson ME, Rocco SE, Sutherland KM, Parsell DA, Mak J, and Grove $\mathrm{BH}$ (1999) Relaxin stimulates expression of vascular endothelial growth factor in normal human endometrial cells in vitro and is associated with menometrorrhagia in women. Hum Reprod 14:800-806.

Unemori EN, Lewis M, Constant J, Arnold G, Grove BH, Normand J, Deshpande U, Salles A, Pickford LB, Erikson ME, et al. (2000) Relaxin induces vascular endothelial growth factor expression and angiogenesis selectively at wound sites. Wound Repair Regen 8:361-370.

Unemori EN, Pickford LB, Salles AL, Piercy CE, Grove BH, Erikson ME, and Amento EP (1996) Relaxin induces an extracellular matrix-degrading phenotype in human lung fibroblasts in vitro and inhibits lung fibrosis in a murine model in vivo. J Clin Invest 98:2739-2745.

van der Westhuizen ET (2008) Molecular characterisation of human and mouse relaxin-3 receptors (RXFP3) in recombinant and endogenously expressing cell lines. Ph.D. Thesis, Monash University, Melbourne, Australia.

van der Westhuizen ET, Christopoulos A, Sexton PM, Wade JD, and Summers RJ (2010) H2 relaxin is a biased ligand relative to H3 relaxin at the relaxin family peptide receptor 3 (RXFP3). Mol Pharmacol 77:759-772.
Van der Westhuizen ET, Sexton PM, Bathgate RA, and Summers RJ (2005) Responses of GPCR135 to human gene 3 (H3) relaxin in CHO-K1 cells determined by microphysiometry. Ann N Y Acad Sci 1041:332-337.

van der Westhuizen ET, Werry TD, Sexton PM, and Summers RJ (2007) The relaxin family peptide receptor 3 activates extracellular signal-regulated kinase 1/2 through a protein kinase C-dependent mechanism. Mol Pharmacol 71:1618-1629.

van Hinsbergh VW, Engelse MA, and Quax PH (2006) Pericellular proteases in angiogenesis and vasculogenesis. Arterioscler Thromb Vasc Biol 26:716-728.

Vasilenko P, Mead JP, and Weidmann JE (1986) Uterine growth-promoting effects of relaxin: a morphometric and histological analysis. Biol Reprod 35:987-995.

Vélez-Ruiz GA and Sunahara RK (2011) Reconstitution of G protein-coupled receptors into a model bilayer system: reconstituted high-density lipoprotein particles. Methods Mol Biol 756:167-182.

Wade JD, Layden SS, Lambert PF, Kakouris H, and Tregear GW (1994) Primate relaxin: synthesis of gorilla and rhesus monkey relaxins. J Protein Chem 13:315-321.

Wang P, Li HW, Wang YP, Chen H, and Zhang P (2009) Effects of recombinant human relaxin upon proliferation of cardiac fibroblast and synthesis of collagen under high glucose condition. J Endocrinol Invest 32:242-247.

Ward DG, Thomas GR, and Cronin MJ (1992) Relaxin increases rat heart rate by a direct action on the cardiac atrium. Biochem Biophys Res Commun 186:999-1005.

Way SA and Leng G (1992) Relaxin increases the firing rate of supraoptic neurones and increases oxytocin secretion in the rat. J Endocrinol 132:149-158.

Weisinger RS, Burns P, Eddie LW, and Wintour EM (1993) Relaxin alters the plasma osmolality-arginine vasopressin relationship in the rat. $J$ Endocrinol 137:505-510.

Weiss F, Ciccocioppo R, Parsons LH, Katner S, Liu X, Zorrilla EP, Valdez GR, BenShahar O, Angeletti S, and Richter RR (2001) Compulsive drug-seeking behavior and relapse. Neuroadaptation, stress, and conditioning factors. Ann N Y Acad Sci 937: $1-26$.

Weiss G (1989) Relaxin in the male. Biol Reprod 40:197-200.

Weiss G, Teichman S, Stewart D, Nader D, Wood S, and Unemori E (2009) A randomized, double-blind, placebo-controlled trial of relaxin for cervical ripening in post-delivery date pregnancies. Ann N Y Acad Sci 1160:385-386.

Whitmarsh AJ and Davis RJ (1996) Transcription factor AP-1 regulation by mitogenactivated protein kinase signal transduction pathways. $J$ Mol $\mathrm{Med}$ (Berl) $\mathbf{7 4}$ 589-607.

Whittaker PG, Edwards JR, Randolph C, Büllesbach EE, Schwabe C, and Steinetz BG (2003) Abnormal relaxin secretion during pregnancy in women with type 1 diabetes. Exp Biol Med (Maywood) 228:33-40.

Whorton MR, Bokoch MP, Rasmussen SG, Huang B, Zare RN, Kobilka B, and Sunahara RK (2007) A monomeric G protein-coupled receptor isolated in a high-density lipoprotein particle efficiently activates its G protein. Proc Natl Acad Sci USA 104:7682-7687.

Wiedemar N, Tetens J, Jagannathan V, Menoud A, Neuenschwander S, Bruggmann $\mathrm{R}$, Thaller $\mathrm{G}$, and Drögemüller C (2014) Independent polled mutations leading to complex gene expression differences in cattle. PLoS ONE 9:e93435.

Wilkinson TN, Speed TP, Tregear GW, and Bathgate RA (2005a) Coevolution of the relaxin-like peptides and their receptors. Ann N Y Acad Sci 1041:534-539.

Wilkinson TN, Speed TP, Tregear GW, and Bathgate RA (2005b) Evolution of the relaxin-like peptide family. BMC Evol Biol 5:14.

Willoughby D, Baillie GS, Lynch MJ, Ciruela A, Houslay MD, and Cooper DM (2007) Dynamic regulation, desensitization, and cross-talk in discrete subcellular microdomains during $\beta_{2}$-adrenoceptor and prostanoid receptor cAMP signaling. $J$ Biol Chem 282:34235-34249.

Winslow JW, Shih A, Bourell JH, Weiss G, Reed B, Stults JT, and Goldsmith LT (1992) Human seminal relaxin is a product of the same gene as human lutea relaxin. Endocrinology 130:2660-2668.

Wu B, Chien EY, Mol CD, Fenalti G, Liu W, Katritch V, Abagyan R, Brooun A, Wells P, Bi FC, et al. (2010) Structures of the CXCR4 chemokine GPCR with smallmolecule and cyclic peptide antagonists. Science 330:1066-1071.

Xiao J, Huang Z, Chen CZ, Agoulnik IU, Southall N, Hu X, Jones RE, Ferrer M Zheng W, Agoulnik AI, et al. (2013) Identification and optimization of smallmolecule agonists of the human relaxin hormone receptor RXFP1. Nat Commun 4 1953

Xu Q, Chakravorty A, Bathgate RA, Dart AM, and Du XJ (2010) Relaxin therapy reverses large artery remodeling and improves arterial compliance in senescent spontaneously hypertensive rats. Hypertension 55:1260-1266.

Xu Q, Lekgabe ED, Gao XM, Ming Z, Tregear GW, Dart AM, Bathgate RA, Samue $\mathrm{CS}$, and Du XJ (2008) Endogenous relaxin does not affect chronic pressure overload-induced cardiac hypertrophy and fibrosis. Endocrinology 149:476-482.

Yamamoto H, Arai T, Tasaka R, Mori Y, Iguchi K, Unno K, and Hoshino M (2009) Inhibitory effect of rlaxin-3 on insulin secretion in isolated pancreas and insulinoma. $J$ Health Sci 55:132-137.

Yamamoto T, Davis CG, Brown MS, Schneider WJ, Casey ML, Goldstein JL, and Russell DW (1984) The human LDL receptor: a cysteine-rich protein with multiple Alu sequences in its mRNA. Cell 39:27-38.

Yan Y, Scott DJ, Wilkinson TN, Ji J, Tregear GW, and Bathgate RA (2008) Identification of the N-linked glycosylation sites of the human relaxin receptor and effect of glycosylation on receptor function. Biochemistry 47:6953-6968.

Yegorov S, Good-Avila SV, Parry L, and Wilson BC (2009) Relaxin family genes in humans and teleosts. Ann N Y Acad Sci 1160:42-44.

Ying SY, Becker A, Baird A, Ling N, Ueno N, Esch F, and Guillemin R (1986) Type beta transforming growth factor (TGF-beta) is a potent stimulator of the basal secretion of follicle stimulating hormone (FSH) in a pituitary monolayer system. Biochem Biophys Res Commun 135:950-956.

Yki-Järvinen H, Wahlström T, and Seppälä M (1983) Immunohistochemical demonstration of relaxin in the genital tract of men. J Reprod Fertil 69:693-695

Yoshida T, Kumagai H, Kohsaka T, and Ikegaya N (2013) Relaxin protects against renal ischemia-reperfusion injury. Am J Physiol Renal Physiol 305:F1169-F1176.

Zarreh-Hoshyari-Khah MR, Einspanier A, and Ivell R (1999) Differential splicing and expression of the relaxin-like factor gene in reproductive tissues of the marmoset monkey (Callithrix jacchus). Biol Reprod 60:445-453. 
Zhang J, Qi YF, Geng B, Pan CS, Zhao J, Chen L, Yang J, Chang JK, and Tang CS (2005) Effect of relaxin on myocardial ischemia injury induced by isoproterenol. Peptides 26:1632-1639.

Zhang Q, Liu SH, Erikson M, Lewis M, and Unemori E (2002) Relaxin activates the MAP kinase pathway in human endometrial stromal cells. J Cell Biochem 85:536-544.

Zhang S, Hughes RA, Bathgate RA, Shabanpoor F, Hossain MA, Lin F, van Lierop B, Robinson AJ, and Wade JD (2010) Role of the intra-A-chain disulfide bond of insulin-like peptide 3 in binding and activation of its receptor, RXFP2. Peptides 31:1730-1736.

Zhang WJ, Wang XY, Guo YQ, Luo X, Gao XJ, Shao XX, Liu YL, Xu ZG, and Guo ZY (2014) The highly conserved negatively charged Glu141 and Asp145 of the G-protein-coupled receptor RXFP3 interact with the highly conserved positively charged arginine residues of relaxin-3. Amino Acids 46:1393-1402.

Zhang X, Zhu M, Zhao M, Chen W, Fu Y, Liu Y, Liu W, Zhang B, Yin X, and Bai B (2013) The plasma levels of relaxin-2 and relaxin-3 in patients with diabetes. Clin Biochem 46:1713-1716.

Zhao L, Roche PJ, Gunnersen JM, Hammond VE, Tregear GW, Wintour EM, and Beck F (1999) Mice without a functional relaxin gene are unable to deliver milk to their pups. Endocrinology 140:445-453.
Zhao L, Samuel CS, Tregear GW, Beck F, and Wintour EM (2000) Collagen studies in late pregnant relaxin null mice. Biol Reprod 63:697-703.

Zhao S, Fields PA, and Sherwood OD (2001) Evidence that relaxin inhibits apoptosis in the cervix and the vagina during the second half of pregnancy in the rat. Endocrinology 142:2221-2229.

Zhao S, Kuenzi MJ, and Sherwood OD (1996) Monoclonal antibodies specific for rat relaxin. IX. Evidence that endogenous relaxin promotes growth of the vagina during the second half of pregnancy in rats. Endocrinology 137:425-430.

Zhu J, Kuei C, Sutton S, Kamme F, Yu J, Bonaventure P, Atack J, Lovenberg TW, and Liu C (2008) Identification of the domains in RXFP4 (GPCR142) responsible for the high affinity binding and agonistic activity of INSL5 at RXFP4 compared to RXFP3 (GPCR135). Eur J Pharmacol 590:43-52.

Zimmermann S, Schöttler P, Engel W, and Adham IM (1997) Mouse Leydig insulinlike (Ley I-L) gene: structure and expression during testis and ovary development. Mol Reprod Dev 47:30-38.

Zimmermann S, Steding G, Emmen JM, Brinkmann AO, Nayernia K, Holstein AF, Engel W, and Adham IM (1999) Targeted disruption of the Insl3 gene causes bilateral cryptorchidism. Mol Endocrinol 13:681-691. 